PALEO

Revue d'archéologie préhistorique

$20 \mid 2008$

Spécial table ronde ( $2^{\mathrm{e}}$ partie) : Le Gravettien : entités régionales d'une paléoculture européenne, Les Eyzies, juillet 2004

\title{
L'« art » pariétal gravettien en France : éléments pour un bilan chronologique
}

Gravettian rock-"art" in France: data for a chronological up dating

Jacques Jaubert

\section{OpenEdition}

Journals

Édition électronique

URL : http://journals.openedition.org/paleo/1635

DOI : $10.4000 /$ paleo. 1635

ISSN : 2101-0420

Éditeur

SAMRA

Édition imprimée

Date de publication : 1 décembre 2008

Pagination : 439-474

ISSN : 1145-3370

Référence électronique

Jacques Jaubert, «L'« art » pariétal gravettien en France : éléments pour un bilan chronologique », PALEO [En ligne], 20 | 2008, mis en ligne le 13 mai 2011, consulté le 07 juillet 2020. URL : http:// journals.openedition.org/paleo/1635; DOI : https://doi.org/10.4000/paleo.1635

\section{(c) (i) (9)}

PALEO est mis à disposition selon les termes de la licence Creative Commons Attribution - Pas d'Utilisation Commerciale - Pas de Modification 4.0 International. 


\title{
L'“ART» PARIÉTAL GRAVETTIEN EN FRANCE : ÉLÉMENTS POUR UN BILAN CHRONOLOGIQUE
}

\author{
Jacques JAUBERT ${ }^{(1)}$
}

\begin{abstract}
Résumé : À l'invitation des responsables du colloque Le Gravettien, entités régionales d'une paléoculture européenne tenu aux Eyzies en juillet 2004, nous proposons un bilan des manifestations pariétales attribuées, attribuables ou proches chronologiquement du Gravettien. La documentation réunie ne concerne que la France et permet de constater une certaine richesse documentaire, un ensemble de sites (ou portion de cavités) assez bien daté, même si les critères d'attribution chronologique diffèrent : datations directes sur pigments pariétaux, datations indirectes d'éléments associés, rapprochement culturel par association de techniques spécifiques, de standards thématiques ou stylistiques.
\end{abstract}

Mots-clés : art pariétal, Gravettien, datations radiocarbone, France, Pléniglaciaire récent.

\begin{abstract}
Titre anglais, Titre anglais, Titre anglais, Titre anglais, Titre anglais, Titre anglais, Following an invitation by the organizers of the conference entitled "Le Gravettien, entités régionales d'une paléoculture européenne" and held in Les Eyzies, France during July 2004, we provide a summary of the expressions of art culturally and chronologically attributed to the Gravettian. The summarized material is restricted to France and establishes a high level of documentary detail for a collection of well-dated sites (or portions of caves), despite the fact that these cultural attributions are based on a variety of chronological criteria: directly dated pigments from paintings, indirect dating via associated elements, cultural attributions based on specific manufacturing techniques or stylistic or thematic standards.
\end{abstract}

Key-words : Rock Art, Gravettian, Radiocarbon dating, France, Last Pleniglacial.

\section{L’ “ART» PARIÉTAL GRAVETTIEN}

II n'est pas question ici de traiter de l'Art pariétal gravettien mais seulement de concentrer le propos sur quelques éléments qui le caractérisent. Le fil conducteur principal sera concentré sur les critères (datations, contexte archéologique, techniques, thématique, bestiaire, conventions, styles...) qui ont servi ou peuvent servir sur la base des dernières études ou résultats d'analyses à attribuer telle ou telle portion de sanctuaire orné à l'ensemble des manifestations du Paléolithique supérieur que les préhistoriens nomment habituellement Gravettien.

Le recours aux guillemets encadrant le terme " art " trouve ici son explication car, dans quelques cas sortant de l'ordinaire, si les parois ou des éléments d'une cavité (pendants rocheux, formations calcitiques...) conservent des vestiges de manifestations dites « pariétales ", leur nature parfois limitée à de simples marques, des séries de ponctuations, etc. peuvent échapper à la notion de valeur artistique telle que l'entend l'historien de l'art, évoquant un autre domaine que celui du décor (notion de grotte dite " ornée ") ou une quelconque manifestation artistique. L'expression « sanctuaire orné » n'en prend alors que plus de force.

\section{Un art assez bien documenté}

L'art pariétal gravettien est assez bien documenté, voire abondant dans certaines régions comme le Quercy ou pour les quelques sanctuaires isolés de la moitié nord de la France (fig. 1). Dans un récent ouvrage de synthèse, Lorblanchet (1995) attribue, sur la foi des datations, dix sites au "Périgordien 》 (= Gravettien). Sur une échelle quantitative, il devancerait à n'en pas douter ceux attribués

(1) Université Bordeaux 1, UMR 5199, PACEA, IPGQ, Avenue des Facultés, 33405 Talence cedex j.jaubert@ipgq.u-bordeaux1.fr 


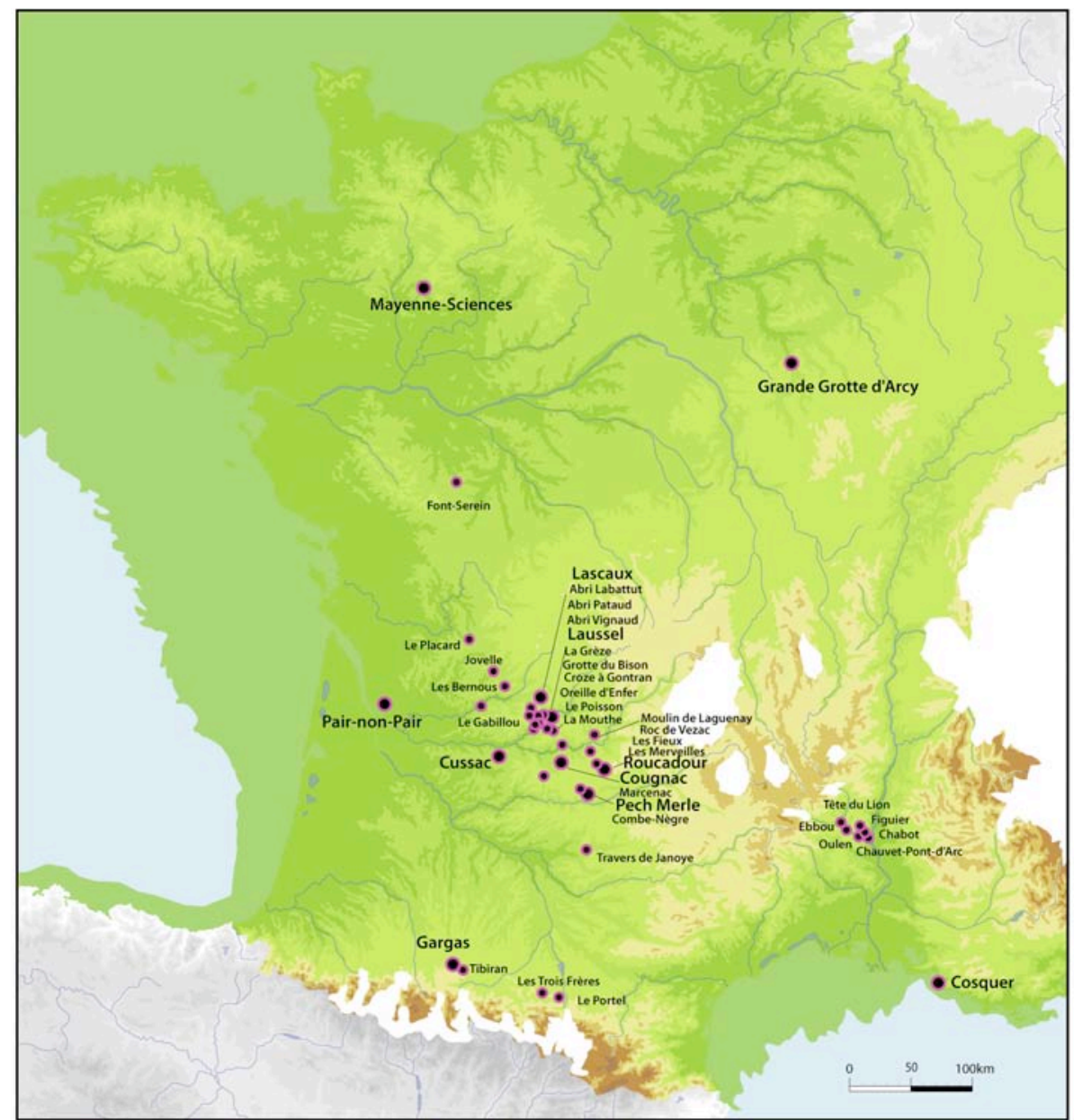

Figure 1 - Carte des sites mentionnés dans le texte. Cf. Légende séparée. (DAO F. Lagarde, J. Jaubert).

Figure 1 - Sites location

Gravettien daté, quasi-certain

- Pigment pariétal daté $\square$ Mouchage daté

- Os au sol daté

$\Delta$ Os fiché daté

O charbon au sol daté * Paroi ornée en stratigraphie

x Bloc orné en stratigraphie

- Date US "habitat"

- $\bigcirc 1$ Cosquer (Marseille, Bouches-du-Rhône)

- 2 Pech-Merle (Cabrerets, Lot)

- 3 Cougnac (Payrignac, Lot)

- 4 Mayenne-Sciences (Thorigné-en-Charnie, Mayenne)

- 5 Gargas inférieure (Aventignan, Hautes-Pyrénées)

ㅇ 7 Grande Grotte d'Arcy (Arcy-sur-Cure, Yonne)

- 8 Cussac (Le Buisson-de-Cadouin, Dordogne)

ㅇ 9 Chauvet-Pont d'Arc (Vallon-Pont d'Arc, Ardèche)

* 10 Pair-non-Pair (Marcamps, Gironde)

* 11 Oreille d'Enfer (Les Eyzies-de-Tayac, Dordogne)

* 12 Labattut (Sergeac, Dordogne)

* 13 Laussel (Marquay, Dordogne)

* 14 Pataud (Les Eyzies-de-Tayac, Dordogne)

× 15 Vignaud (Les Eyzies-de-Tayac, Dordogne)

\section{$\infty$ Gravettien très probable}

$\infty \quad 6$ Tibiran (Tibiran-Jaunac, Hautes-Pyrénées)

$\infty \quad 16$ Roucadour (Thémines, Lot)

$\infty \quad 17$ Les Merveilles (Rocamadour, Lot) $\infty \quad 18 \quad$ Les Fieux (Miers, Lot)

$\infty \quad 19$ Marcenac (Cabrerets, Lot)

$\infty \quad 20$ Combe-Nègre (Frayssinet-le-Gélat, Lot)

$\infty 21$ Roc de Vézac (Vézac, Dordogne)

$\infty \quad 22$ Le Bison (Meyrals, Dordogne)

$\infty \bigcirc 23$ Moulin de Laguenay (Chasteaux, Corrèze)

$\infty \quad 26 \quad$ Le Portel (Loubens, Ariège)

$\infty \quad 27$ Les Trois Frères, Enlène

(Montesquieu-Avantès, Ariège)

$\infty \quad 28$ Le Poisson (Les Eyzies-de-Tayac, Dordogne)

Gravettien discuté ou autres mentions chronologiquement proches

24 Travers de Janoye (Penne, Tarn)

* * 25 Le Placard (Vilhonneur, Charente)

29 La Grèze (Marquay, Dordogne)

30 La Mouthe (Les Eyzies-de-Tayac, Dordogne)

31 La Croze à Gontran (Les Eyzies-de-Tayac,

Dordogne)

32 Les Bernous (Bourdeilles, Dordogne)

33 Jovelle (La Tour-Blanche, Dordogne)

34 Font-Serein (Lussac-les-Châteaux, Vienne)

O 35 Tête-du-Lion (Bidon, Ardèche)

36 Chabot (Aiguèze, Gard)

37 Oulen (Le Garn, Gard)

- 38 Le Figuier (Saint-Martin-d'Ardèche, Ardèche)

39 Ebbou (Vallon-Pont d'Arc, Ardèche)

40 Lascaux (Montignac, Dordogne)

41 Gabillou (Sourzac, Dordogne) 
ou attribuables à l'Aurignacien, serait sensiblement équivalent au corpus solutréen ou assimilé (Cosquer Phase 2) et ne serait distancé que par les phases moyenne et récente du Magdalénien.

Notons dès à présent que le compartimentage reprenant la classification héritée des concepts de Mortillet - Breuil Peyrony - Bordes - de Sonneville-Bordes subdivisant le Paléolithique récent ${ }^{2}$ en Aurignacien - Gravettien Solutréen - Badegoulien - Magdalénien, eux-mêmes tous subdivisés, trouve bien souvent ses limites, notamment quand il s'agit d'art. Preuve en est que, pour l'art pléistocène justement, les principaux auteurs ont bâti des tableaux qui transgressent cette classification : " cycle aurignacopérigordien » et « solutréo-magdalénien » de Breuil (1952), « inter Gravetto-Solutréen » ou « style II » de Leroi-Gourhan (1965), ce dernier couvrant Gravettien et début du Solutréen. Pour ne citer ici que les maîtres disparus.

\section{Un art assez bien étudié}

Sans se livrer à un historique approfondi des travaux consacrés à l'art gravettien, on soulignera que nombre de synthèses régionales ont permis de bâtir les grands référentiels pour le domaine franco-cantabrique. Nous aurions tendance à citer en premier le Quercy de M. Lorblanchet, « très gravettien » par ses deux principaux sites ornés que sont Pech-Merle et Cougnac, complétés par une belle série de cavités moins connues, mais complémentaires (Lorblanchet 1972, 1974, 2004). L'Aquitaine s.s., Périgord en tête, suit de très près, mais, contrairement à son voisin méridional, les cavités emblématiques - à l'exception de Cussac (Aujoulat et al. 2004) - sont généralement attribuées à des périodes encadrant le Gravettien, Solutréen et surtout Magdalénien. La densité documentaire est cependant reconnue et bien répertoriée pour cet art qualifié parfois d'archaïque (Delluc et Delluc 1991). On mentionnera également l'Ardèche longtemps référencée grâce aux travaux de J. Combier et al. 1960.

Une extension géographique d'importance permet de gagner le piémont nord pyrénéen avec Gargas (Barrière 1976), des secteurs des Cavernes du Volp (Bégouën et Breuil 1958) ou du Portel, le littoral provençal et Cosquer (Clottes et Courtin 1994 ; Clottes, Courtin et Vanrell 2005), les confins de la Bourgogne avec la Grande Grotte d'Arcy (Baffier et Girard 1998) et le Maine avec MayenneSciences (Pigeaud 2001).

Les publications ne manquent pas et, poursuivant les travaux précurseurs pour ne pas dire visionnaires de Breuil (1952), puis de Leroi-Gourhan (1965), des synthèses plus ou moins détaillées les ont nuancés ou réactualisés (e.g.
Clottes 1995, 2000 ; Combier 1989 ; Lorblanchet 1995 ; Sauvet 2004 , etc.). La présente contribution a donc été grandement facilitée par une documentation publiée abondante bien qu'inégale.

\section{Un art assez bien daté}

Nous ne disposons - et ne disposerons jamais - d'assez de dates, relatives ou " directes » (« objectives » pour Lorblanchet 1994). Ceci dit, les âges radiocarbone, tous non calibrés (Bard et al. 1998), concernant peu ou prou des sanctuaires supposés gravettiens existent et sont même assez nombreux, plus d'une soixantaine (tabl. 1) mais leur statut diffère (tabl. 2) :

- une quinzaine d'âges BP directs seulement, véritables datations d'éléments composant l'entité graphique (charbons, fraction humique) : 8 à Cosquer, 4 à Cougnac, 2 à Mayenne-Sciences, 1 à Pech-Merle ;

- une seule date a été obtenue sur un échantillon osseux intégré au dispositif pariétal, un os fiché (Gargas) ;

- une dizaine de dates participent de près à ce que l'on estime par analogie comme possiblement associé au dispositif orné, charbons ou os au sol de la Grande Grotte d'Arcy (8), Cosquer (2) et probable sépulture de Cussac (1) ;

- enfin, des datations participant « de loin » au dispositif pariétal, par exemple un niveau archéologique attesté dans un secteur voisin du sanctuaire : Enlène pour les Trois-Frères, Mayenne-Sciences, Les Fieux et Pataud ;

- le cas de Chauvet est particulier avec des datations de mouchages de torche (4) attribués au Gravettien - d'ailleurs les seules - charbons ou foyers au sol (10).

Quand le contexte gravettien était prégnant, nous avons retenu quelques dates qui sortent quelque peu des limites admises pour le Gravettien, mordant soit sur l'Aurignacien récent (Arcy), soit sur le Solutréen (Cougnac) ${ }^{3}$.

\section{1 - SANCTUAIRES ORNÉS GRAVETTIENS QUASI- CERTAINS : DATATIONS RADIONUMÉRIQUES DIRECTES ET INDIRECTES}

La présentation qui suit mentionne des sanctuaires ou portions de sanctuaires datés par des méthodes radionucléaires. Encore convient-il de rappeler que nous entendons par date ${ }^{14} \mathrm{C}$ un âge ${ }^{14} \mathrm{C}$ non calibré de l'échantillon prélevé et non par association automatique de l'unité graphique. En cela, la date obtenue peut être différente de l'âge de réalisation de l'unité graphique, et de toutes manières de la date calendaire, la calibration n'étant pas encore systématiquement applicable pour ces périodes. C'est donc la somme de concordances, le phénomène de

(2) Nous avons expliqué notre préférence pour « récent » plutôt que « supérieur » : Jaubert J., 2003. Les archéologues travaillant sur des périodes antérieures au Paléolithique supérieur appartiennent-ils à une espèce (ou sous-espèce) différente ? In : Sens dessus dessous. La recherche du sens en préhistoire. Recueil d'études offert à Jean Leclerc \& Claude Masset. Revue archéo. de Picardie, p. 69-74.

(3) Pour l'interprétation des dates obtenues en art pariétal, on se reportera également à Sauvet, 2004. 


\begin{tabular}{|c|c|c|c|c|c|c|}
\hline Site & Commune & Département & Secteur - UG & Topo & Éch. Daté & Méthode \\
\hline COSQUER & Marseille & Bouches-du-Rh. & Signe ovale secteur 201 & Paroi & charbon & SMA \\
\hline COSQUER & Marseille & Bouches-du-Rh. & Sol félin secteur 117 & Sol & charbon & SMA \\
\hline COSQUER & Marseille & Bouches-du-Rh. & Main M19 secteur 205 & Paroi & charbon & SMA \\
\hline COSQUER & Marseille & Bouches-du-Rh. & Bison 2 secteur 108 & Paroi & charbon & SMA \\
\hline COSQUER & Marseille & Bouches-du-Rh. & Main MR7 secteur 117 & Paroi & charbon & SMA \\
\hline COSQUER & Marseille & Bouches-du-Rh. & Main MR7 secteur 117 & Paroi & charbon & SMA \\
\hline COSQUER & Marseille & Bouches-du-Rh. & Sol pingouin secteur 204 & Sol & charbon & SMA \\
\hline COSQUER & Marseille & Bouches-du-Rh. & Bison 2 secteur 108 & Paroi & charbon & SMA \\
\hline COSQUER & Marseille & Bouches-du-Rh. & Main & Paroi & acide humique & SMA \\
\hline COSQUER & Marseille & Bouches-du-Rh. & Main M12 secteur 205 & Paroi & charbon & SMA \\
\hline COSQUER & Marseille & Bouches-du-Rh. & Cheval 5 secteur 101 & Paroi & charbon & SMA \\
\hline PECH-MERLE & Cabrerets & Lot & Chevaux ponctués & Paroi & charbon & SMA \\
\hline Pech-Merle & Cabrerets & Lot & Sol Chevaux ponctués & Sol & Os & \\
\hline COUGNAC & Payrignac & Lot & Mégacéros femelle (bosse) & Paroi & charbon & SMA \\
\hline COUGNAC & Payrignac & Lot & Mégacéros mâle & Paroi & charbon & SMA \\
\hline COUGNAC & Payrignac & Lot & Mégacéros mâle & Paroi & charbon & SMA \\
\hline COUGNAC & Payrignac & Lot & Mégacéros femelle (dos) & Paroi & charbon & SMA \\
\hline MAYENNE-SCIENCES & Thorigné-en-Charnie & Mayenne & Cheval 15 (dos) & Paroi & charbon & SMA \\
\hline MAYENNE-SCIENCES & Thorigné-en-Charnie & Mayenne & Cheval 15 (genou) & Paroi & charbon & SMA \\
\hline MAYENNE-SCIENCES & Thorigné-en-Charnie & Mayenne & Entrée & Entrée & $1,5 \mathrm{~kg} \mathrm{os}$ & classique \\
\hline Chauvet Pont-d'Arc & Vallon-Pont-d'Arc & Ardèche & Salle des Croisillons & Tracé chinois & charbon & $S M A$ \\
\hline Chauvet Pont-d'Arc & Vallon-Pont-d'Arc & Ardèche & Salle Hillaire ${ }^{* *}$ & Mouchage & charbon & $S M A$ \\
\hline Chauvet Pont-d'Arc & Vallon-Pont-d'Arc & Ardèche & Salle Hillaire ** & Mouchage & charbon & $S M A$ \\
\hline Chauvet Pont-d'Arc & Vallon-Pont-d'Arc & Ardèche & Salle du Cierge GCA & Sol /s stalagm. & charbon & SMA \\
\hline Chauvet Pont-d'Arc & Vallon-Pont-d'Arc & Ardèche & Galerie des Mégacéros & Sol & charbon & SMA \\
\hline Chauvet Pont-d'Arc & Vallon-Pont-d'Arc & Ardèche & Salle des Panneaux rouges* & Sol & charbon & SMA \\
\hline Chauvet Pont-d'Arc & Vallon-Pont-d'Arc & Ardèche & Salle des Panneaux rouges* & Sol & charbon & SMA \\
\hline Chauvet Pont-d'Arc & Vallon-Pont-d'Arc & Ardèche & Salle du Cierge & Foyer & charbon & SMA \\
\hline Chauvet Pont-d'Arc & Vallon-Pont-d'Arc & Ardèche & Salle Hillaire ${ }^{* * *}$ & Mouchage & charbon & $S M A$ \\
\hline Chauvet Pont-d'Arc & Vallon-Pont-d'Arc & Ardèche & Salle des Croisillons & Sol & charbon & SMA \\
\hline Chauvet Pont-d'Arc & Vallon-Pont-d'Arc & Ardèche & Galerie des Mégacéros & Encr.carb. & charbon & SMA \\
\hline Chauvet Pont-d'Arc & Vallon-Pont-d'Arc & Ardèche & Salle Hillaire & Foyer & charbon & SMA \\
\hline Chauvet Pont-d'Arc & Vallon-Pont-d'Arc & Ardèche & Salle du Fond & Sol & charbon & SMA \\
\hline Chauvet Pont-d'Arc & Vallon-Pont-d'Arc & Ardèche & Salle du Fond & Sol & charbon & SMA \\
\hline GARGAS inférieure & Aventignan & Hautes-Pyrénées & Grande Paroi des mains & Paroi & os & $S M A$ \\
\hline Gargas inférieure & Aventignan & Hautes-Pyrénées & & & bois de renne & $S M A$ \\
\hline GRANDE GROTTE & Arcy-s/-Cure & Yonne & & Sol & os & SMA \\
\hline GRANDE GROTTE & Arcy-s/-Cure & Yonne & & Sol & os & SMA \\
\hline GRANDE GROTTE & Arcy-s/-Cure & Yonne & Salle des Vagues & Sol & charbon & SMA \\
\hline GRANDE GROTTE & Arcy-s/-Cure & Yonne & Corniche au bison & Sol & os brûlé & SMA \\
\hline GRANDE GROTTE & Arcy-s/-Cure & Yonne & Salle des Vagues & Sol & charbon & SMA \\
\hline GRANDE GROTTE & Arcy-s/-Cure & Yonne & Salle des Noyaux de cerise & Mouchage & charbon & $S M A$ \\
\hline GRANDE GROTTE & Arcy-s/-Cure & Yonne & Sol Frise rouge & Sol & os brûlé & SMA \\
\hline GRANDE GROTTE & Arcy-s/-Cure & Yonne & Salle des Noyaux de cerise & Mouchage & charbon & SMA \\
\hline GRANDE GROTTE & Arcy-s/-Cure & Yonne & Sol Frise rouge & Sol & os brûlé & SMA \\
\hline GRANDE GROTTE & Arcy-s/-Cure & Yonne & Sol paroi peinte & Sol & os brûlé & SMA \\
\hline CUSSAC & Buisson-de-Cadouin & Dordogne & Sépulture locus 1 & Sol & os humain & SMA \\
\hline Les Fieux & Miers & Lot & Porche Est, c. Flc & n. archéo. & os & classique \\
\hline Enlène & Montesquieu-Avantès & Ariège & $E D G$ c. 5 & n. archéo. & métap. Cerf & $S M A$ \\
\hline Enlène & Montesquieu-Avantès & Ariège & EDG c. 5 & n. archéo. & os & classique \\
\hline Enlène & Montesquieu-Avantès & Ariège & $E D G c .4$ & n. archéo. & os & classique \\
\hline Abri Pataud & Les Eyzies-de-Tayac & Dordogne & Niveau 3 Grav. récent 3 & n.archéo. & os acide am. & \\
\hline Abri Pataud & Les Eyzies-de-Tayac & Dordogne & Niveau 3 Grav. récent 3 & n.archéo. & os acide am. & \\
\hline Abri Pataud & Les Eyzies-de-Tayac & Dordogne & Niveau 3 Grav récent $2-2 a$ & n.archéo. & os acide am. & \\
\hline Abri Pataud & Les Eyzies-de-Tayac & Dordogne & Niveau 3 Grav récent $2-2 a$ & n.archéo. & os acide am. & \\
\hline Abri Pataud & Les Eyzies-de-Tayac & Dordogne & Niveau 3 Grav récent $2-2 a$ & n.archéo. & os ext.collag. & \\
\hline Abri Pataud & Les Eyzies-de-Tayac & Dordogne & Niveau 3 Grav récent $2-2 a$ & n.archéo. & os ext.collag. & classique \\
\hline Abri Pataud & Les Eyzies-de-Tayac & Dordogne & Niveau 3 Grav récent $2-2 a$ & n.archéo. & os ext.collag. & classique \\
\hline Abri Pataud & Les Eyzies-de-Tayac & Dordogne & Niveau 3 Grav récent $2-2 a$ & n.archéo. & os brûlé & classique \\
\hline Abri Pataud & Les Eyzies-de-Tayac & Dordogne & Niveau 3 Grav récent $2-2 a$ & n.archéo. & os brîlé & classique \\
\hline Abri Pataud & Les Eyzies-de-Tayac & Dordogne & Niveau 2 Grav. final 2 & n.archéo. & os acide am. & \\
\hline Abri Pataud & Les Eyzies-de-Tayac & Dordogne & Niveau 2 Grav final 2 & n.archéo. & os fract. rés. & classique \\
\hline Abri Pataud & Les Eyzies-de-Tayac & Dordogne & Niveau 2 Grav final 2 & n.archéo. & os ext.collag. & classique \\
\hline Abri Pataud & Les Eyzies-de-Tayac & Dordogne & Niveau 2 Grav final 2 & n.archéo. & os brûlé & classique \\
\hline Abri Pataud & Les Eyzies-de-Tayac & Dordogne & Niveau 2 Grav final 2 & n.archéo. & os ext.collag. & classique \\
\hline Abri Pataud & Les Eyzies-de-Tayac & Dordogne & Niveau 2 Grav final 2 & n.archéo. & os brûlé & classique \\
\hline Abri Pataud & Les Eyzies-de-Tayac & Dordogne & Niveau 2 Grav final 2 & n.archéo. & os fract. rés. & classique \\
\hline Abri Pataud & Les Eyzies-de-Tayac & Dordogne & Niveau 2 Grav final 2 & n.archéo. & os ext.collag. & classique \\
\hline Abri Pataud & Les Eyzies-de-Tayac & Dordogne & Niveau 2 Grav final 2 & n.archéo. & os ext.collag. & classique \\
\hline Abri Pataud & Les Eyzies-de-Tayac & Dordogne & Niveau 2 Grav final 2 & n.archéo. & os ext.collag. & classique \\
\hline Abri Pataud & Les Eyzies-de-Tayac & Dordogne & Niveau 2 Grav final 2 & n.archéo. & os brûlé & classique \\
\hline
\end{tabular}




\begin{tabular}{|c|c|c|c|}
\hline Âge BP & 1 sigma & Référence & Référence bibliographique \\
\hline 28370 & \pm 440 & Gif A 96074 & Valladas et al., 2005 \\
\hline 27870 & \pm 470 & Gif A 92350 & Clottes et al. 1992 \\
\hline 27740 & \pm 410 & Gif A 96073 & Valladas et al., 2005 \\
\hline 27350 & \pm 430 & Gif A 95195 & Valladas et al., 2005 \\
\hline 27110 & \pm 390 & Gif A 92409 & Clottes et al. 1992 \\
\hline 27110 & \pm 350 & Gif A 92491 & Clottes, Courtin, 1994 \\
\hline 26360 & \pm 440 & Gif A 95349 & Clottes et al. 1992 \\
\hline 26250 & \pm 350 & Gif A 96069 & Valladas et al., 2005 \\
\hline 26180 & $\pm \mathbf{3 3 0}$ & GifA 92424 & Clottes et al. 1992 \\
\hline 24840 & \pm 340 & Gif A 95358 & Valladas et al., 2005 \\
\hline 24730 & $\pm \mathbf{3 0 0}$ & Gif A 9672 & Valladas et al., 2005 \\
\hline 24640 & \pm 390 & Gif A 95357 & Lorblanchet et al., 1995 \\
\hline 18400 & \pm 350 & & Lorblanchet, 1995 \\
\hline 25120 & \pm 390 & Gif A 92425 & Lorblanchet et al., 1990 \\
\hline 23610 & \pm 350 & Gif A 91183 & Lorblanchet et al., 1990 \\
\hline 22750 & \pm 390 & Gif A 92426 & Lorblanchet et al., 1990 \\
\hline 19500 & \pm 270 & Gif A 91324 & Lorblanchet et al., 1990 \\
\hline 24900 & \pm 360 & Gif A 100645 & Pigeaud et al. 2003 \\
\hline 24220 & \pm 850 & Gif A 100647 & Pigeaud et al. 2003 \\
\hline 22600 & \pm 380 & Gif 7714 & Bigot 1988 \\
\hline 27130 & \pm 490 & Gif A 101454 & Valladas et al., 2005 \\
\hline 26980 & \pm 420 & Gif A 95130 & Valladas et al., 2001 \\
\hline 26980 & \pm 410 & Gif A 95129 & Valladas et al., 2001 \\
\hline 26590 & \pm 300 & Gif A 102572 & Valladas et al., 2005 \\
\hline 26590 & \pm 300 & Gif A 99777 & Valladas et al., 2001 \\
\hline 26360 & \pm 290 & Gif A 102569 & Valladas et al., 2005 \\
\hline 26250 & \pm 280 & Gif A 102570 & Valladas et al., 2005 \\
\hline 26230 & \pm 280 & Gif A 99081 & Valladas et al., 2001 \\
\hline 26120 & \pm 400 & Gif A 95127 & Valladas et al., 2001 \\
\hline 26100 & \pm 330 & Gif A 101457 & Valladas et al., 2005 \\
\hline 25440 & \pm 250 & Gif A 99237 & Valladas et al., 2001 \\
\hline 24770 & \pm 780 & Lyon-118/OxA & Évin, Oberlin, 2001 \\
\hline 24240 & \pm 280 & Ly-9381 & Valladas et al., 2005 \\
\hline 22800 & \pm 400 & Ly-6879 & Évin, Oberlin, 2001 \\
\hline 26860 & \pm 460 & Gif $A 92369$ & Clottes et al., 1992 \\
\hline 25050 & \pm 170 & & Foucher, 2004 \\
\hline 30160 & \pm 140 & & Baffier, Girard, 1998 \\
\hline 29640 & \pm 590 & & Baffier, Girard, 1998 \\
\hline 28250 & \pm 430 & Gif A 91370 & Baffier, Girard, 1998 \\
\hline 27950 & \pm 440 & Gif A 95620 & Baffier, Girard, \\
\hline 27630 & \pm 400 & Gif A 92330 & Baffier, Girard, \\
\hline 27080 & \pm 400 & GifA 98185 & Baffier, Girard, 1998 \\
\hline 26700 & \pm 410 & Gif A 94580 & Baffier, Girard, 1995 \\
\hline 26470 & \pm 390 & GifA 98184 & Baffier, Girard, 1998 \\
\hline 26250 & \pm 500 & OxA 5003 & Baffier, Girard, \\
\hline 24660 & \pm 330 & & Baffier, Girard, 1995 \\
\hline 25120 & \pm 120 & Beta 156643 & Aujoulat et al., 2004 \\
\hline 23900 & \pm 330 & Gif 6304 & Champagne, Jaubert, 1986 \\
\hline 27980 & \pm 480 & GifA 97306 & Foucher et al., 2001 \\
\hline 24600 & \pm 350 & Gif 6656 & Clottes, 1989 \\
\hline 21000 & \pm 250 & Gif 6655 & Clottes, 1989 \\
\hline 24500 & \pm 600 & $O x A-686$ & Gowlett et al., 1987 \\
\hline 21740 & \pm 450 & OxA-599 & Gowlett et al., 1987 \\
\hline 24440 & \pm 740 & $O x A-165$ & Gowlett et al., 1987 \\
\hline 24250 & \pm 750 & $O x A-164$ & Gowlett et al., 1987 \\
\hline 23180 & \pm 670 & $O x A-163$ & Gowlett et al., 1987 \\
\hline 23010 & \pm 170 & $G r N-4721$ & Vogel, Waterblock, 1967 \\
\hline 22780 & \pm 140 & $G r N-4506$ & Vogel, Waterblock, 1967 \\
\hline 21540 & \pm 160 & GrN-1892 & Vogel, Waterblock, 1963 \\
\hline 18470 & \pm 280 & $G r N-1864$ & Vogel, Waterblock, 1963 \\
\hline 22000 & \pm 600 & $O x A-162$ & Gowlett et al., 1987 \\
\hline 21940 & \pm 250 & $G r N-1862$ & Vogel, Waterblock, 1963 \\
\hline 21380 & \pm 340 & $G r N-4231$ & Vogel, Waterblock, 1967 \\
\hline 20960 & \pm 220 & $G r N-1857$ & Vogel, Waterblock, 1963 \\
\hline 20810 & \pm 170 & $G r N-4230$ & Vogel, Waterblock, 1967 \\
\hline 20780 & \pm 170 & $G r N-1861$ & Vogel, Waterblock, 1963 \\
\hline 20540 & \pm 140 & $G r N-2081$ & Vogel, Waterblock, 1963 \\
\hline 20340 & \pm 200 & $G r N-2115$ & Vogel, Waterblock, 1963 \\
\hline 19780 & \pm 170 & $G r N-2123$ & Vogel, Waterblock, 1963 \\
\hline 19650 & \pm 300 & GrN-3225 & Vogel, Waterblock, 1967 \\
\hline 19300 & \pm 170 & $G r N-1885$ & Vogel, Waterblock, 1963 \\
\hline
\end{tabular}

Tableau 1 - datations obtenues pour les grottes ou abris ornés attribués ou attribuables au Gravettien.

Table 1 - Dating obtained for caves or rockshelters with rock art related to the Gravettian.

\footnotetext{
* Salle des Bauges in Valladas et al., 2005.

** Salle Hillaire in Valladas et al., 2001 et Salle du Cierge in Valladas et al. 2005 *** Salle du Cierge in Valladas et al. 2001 et Salle Hillaire in Valladas etal., 2005
} 


\begin{tabular}{|c|c|c|c|c|c|c|}
\hline \multirow{14}{*}{ 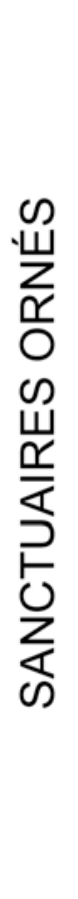 } & \multirow{6}{*}{ 皆 } & DIRECTS & \multirow{6}{*}{ 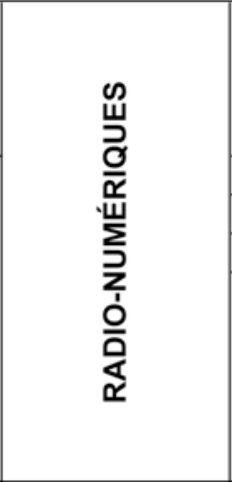 } & Pigment (paroi) & $\begin{array}{l}\text { Cosquer Phase } 1 \\
\text { Cougnac } \\
\text { Mayenne-Sciences } \\
\text { Pech-Merle }\end{array}$ & \\
\hline & & \multirow{5}{*}{ INDIRECTS } & & Os fiché & Gargas inférieure & \\
\hline & & & & Mouchages $^{\star \star}$ & Chauvet** $^{\star \star}$ & \\
\hline & & & & Sépulture (?) & Cussac & \\
\hline & & & & \multirow[t]{2}{*}{ Éléments au sol } & $\begin{array}{l}\text { Grande Grotte d'Arcy } \\
\text { Chauvet } \\
\text { Cosquer Phase } 1\end{array}$ & \\
\hline & & & & & $\begin{array}{l}\text { Cougnac* } \\
\text { Pech-Merle* }\end{array}$ & \\
\hline & \multirow{8}{*}{ 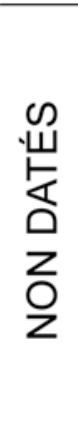 } & \multirow{4}{*}{ TERMINUS A QUO } & \multirow{3}{*}{ RELATIVES } & Paroi ornée scellée & Pair-non-Pair & Gravettien ancien \\
\hline & & & & & Laussel & Gravettlen n.d. \\
\hline & & & & US à éléments ornés & $\begin{array}{l}\text { Labattut } \\
\text { Vignaud }\end{array}$ & Gravettien Noailles \\
\hline & & & & & Pataud & Gravettien final \\
\hline & & & $\begin{array}{c}\text { RADIO- } \\
\text { NUMÉRIQUES }\end{array}$ & & $\begin{array}{l}\text { Gargas inférieure } \\
\text { Enlène / Trois Frères }\end{array}$ & Gravettien Noailles \\
\hline & & INDIRECTS & & US secteur d'habitat & Les Fieux & Gravettien n.d. \\
\hline & & & RELATIVES & & Abri du Poisson & Gravettien Noailles \\
\hline & & & & & & Grav.,Épigr.,Solutr. \\
\hline
\end{tabular}

* Éléments au sol datés mais non gravettiens

*^ Chauvet indiqué mais sanctuaire non gravettien sauf Panneau rouge (?)

Tableau 2 - Critères méthodologiques d'attribution chronologique des abris et grottes ornées au Gravettien.

Table 2 - Methodology criteria for chronological attribution for rock shelters and rock art caves from the Gravettian.

répétition, de recoupements vérifiés qui permettent de valider une ou mieux, les datations physiques.

\section{1 - Dates "directes" de pigments pariétaux (charbon)}

\section{Cosquer (Provence), Phase 1 bis ou 2}

Cet ensemble est désormais suffisamment connu et publié (Clottes et Courtin 1992 ; Clottes, Courtin et Vanrell 2005) pour que l'on y revienne en détail. Rappellons juste qu'il s'agit d'une cavité du littoral provençal, s'ouvrant à -37 m NGF entre Marseille et la calanque de Morgiou. Régression marine ou non, il s'agit d'un sanctuaire profond qui, d'après les relevés serait aujourd'hui noyé aux deux tiers (Collina-Girard 1999).

Les auteurs s'accordent pour distinguer au moins deux périodes de fréquentation (Clottes et al. 1992), la première située entre 28500 et 24500 BP (Gravettien), la seconde entre 19200 et 18500 BP, soit le Solutréen pour Clottes et Courtin et le Tardigravettien pour Brochier et Livache (2003) arguant logiquement que le Solutréen n'a jamais franchi le Rhône. Nous évoquerons plus loin mais succinctement la phase solutréenne ou tardigravettienne qui sort des limites que nous nous sommes fixées.

En fait, la première phase de Cosquer est plutôt une phase 1bis ou 2 (Clottes et al. 2005) dans la mesure où les empreintes de mains négatives datées oblitèrent systématiquement une première génération de tracés digitaux qui, par définition, ne peuvent être directement datés. Clottes et al. 2005 signalent cependant que des tracés digitaux peuvent également surcharger des empreintes de mains et les attribuent de ce fait - comme pour Gargas et les Trois-Frères à la même génération de fréquentation du sanctuaire, le Gravettien. Les dates de cette première phase de Cosquer sont reportées sur le tableau 1, s'échelonnant entre 28370 \pm 440 BP pour le signe ovale du secteur 201 et $24730 \pm 300$ BP pour le cheval 5 du secteur 101 (Valladas et al. 2005). Des gravures « fines » parfois animalières sont localement oblitérées par les pigments d'empreintes de mains confirmant le fait qu'une partie au moins sont à rapporter à la période gravettienne (p. ex. Clottes et al. 2005 - p. 82-83).

Les UG attribuables et attribuées à cette Phase 1 bis ou 2 sont quelques empreintes de mains positives mais surtout négatives, pas moins de 55 dans un premier recensement, empreintes réalisées au pochoir, entières ou avec des doigts « incomplets » ou plutôt repliés (fig. 2), motifs extrêmement intéressant puisque nous retrouvons ce thème si original à Gargas (26 $860 \pm 460 \mathrm{BP})$, dans sa voisine Tibiran, à Arcysur-Cure, mais aussi à Fuente del Salin (22 $340 \pm 510$ BP). 


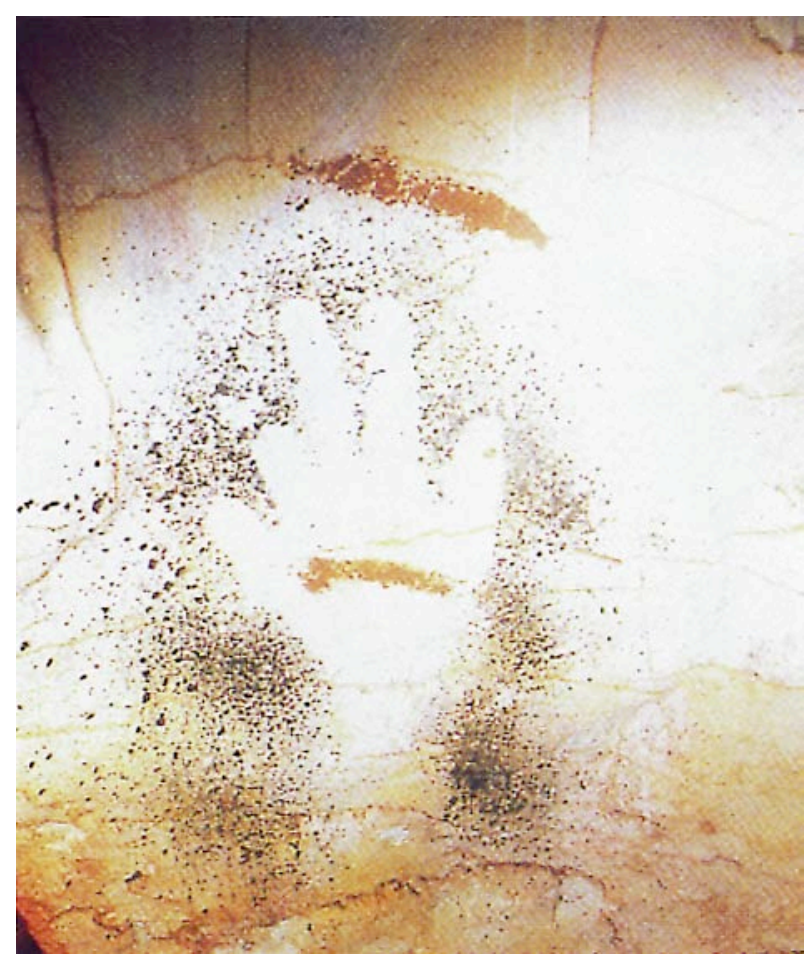

Figure 2 - Grotte Cosquer (Marseille, Bouches-du-Rhône). Empreinte de main négative noire aux doigts incomplets (MR7) ayant fait l'objet de deux datations ${ }^{14} \mathrm{C}$ à $27110 \pm$ 350 et \pm 390 BP (@ Collina-Girard in Clottes et Courtin 1994).

Figure 2 - Cosquer Cave (Marseille, Bouches-du-Rhône). Black hand stencils with 'missing' fingers (MR7) dated to 27,110 \pm 350 and \pm 390 BP (C) Collina-Girard in Clottes \& Courtin 1994).

Concernant la seconde phase, tout l'art animalier de Cosquer n'est pas attribuable " en bloc » au Tardigravettien puisque, au moins les pigments noirs d'un bison ont été directement datés par deux fois à $26250 \pm$ 350 et $27350 \pm 430$ BP (Valladas et al. 2005). Des gravures fines sont également recouvertes par les projections noires d'une empreinte de main (M9). L'homogénéité stylistique des figures animales, qu'elle qu'en soit la technique (dessin peint ou gravé) avait été soulignée dès le début des travaux à Cosquer et la discussion récente publiée à l'occasion de la dernière monographie ne permet pas de trancher entre plusieurs hypothèses qui, d'ailleurs, ne s'excluent pas (Clottes et al. 2005) : homogénéité du bestiaire et des conventions sur les deux périodes, utilisation de charbons « gravettiens » pour dessiner par les auteurs du second passage, pollution des échantillons artificiellement vieillis, etc.

Un élément mérite d'être rappelé : tel qu'il figure, par sa thématique (mégacéros), ses conventions (faiblesse des détails anatomiques, profils absolus, pattes en $X-Y . .$.$) , son$ architecture graphique, le bestiaire de Cosquer ne dépare- rait pas, pro parte, en contexte gravettien. Cela peut jouer également en raison d'une forme de continuité culturelle, technologique et donc artistique désormais acquise entre Gravettien et Solutréen, d'autant qu'ici c'est en plus le Tardigravettien ancien qui est principalement concerné.

De même pour les fréquentations dans la cavité qui présentent le même dédoublement chronologique avec une série de charbons au sol qui pour certains sont attribuables aux Gravettiens (tabl. 1) : échantillons récoltés au sol au droit du félin (secteur 117) daté de $27870 \pm 470$ ainsi qu'au pied du pingouin du secteur 204 daté de $26360 \pm 440$ (Valladas et al. 2005).

\section{Pech-Merle (Quercy)}

Dans cet important sanctuaire quercinois dont l'âge a longtemps été discuté mais toujours rapporté à une phase antérieure au Magdalénien classique (p.ex. Lemozi et al. 1969 ; Leroi-Gourhan 1965 ; Lorblanchet 1984), l'obtention d'une date sur le célèbre panneau des Chevaux ponctués, $24640 \pm 390 \mathrm{BP}$, plus précisément sur le poitrail du cheval de droite (Lorblanchet 1995 ; Lorblanchet et al. 1995) associe désormais cet ensemble au Gravettien (fig. 3). Pour être juste, il convient de rappeler que Pech-Merle avait fourni d'autres résultats que l'on qualifiera pudiquement de « discordants » avec cette dernière date : un charbon recueilli sur le sol, justement à proximité des Chevaux ponctués, avait donné $11380 \pm 390 \mathrm{BP}$; de même, pour un charbon prélevé au pied de la Frise Noire (Chapelle des Mammouths) avec une date aussi jeune, $11200 \pm 800$ BP ; enfin un échantillon provenant d'un sondage, niveau II et un résultat proche, $11460 \pm 390 \mathrm{BP}$ (Lorblanchet 1981, 1992). J. Évin considère comme des âges minimaux et peu fiables les deux premiers dans la mesure où il n'a pu enlever les parties humiques et que les quantités étaient trop faibles (in litt., Lorblanchet 1981 - p. 214-15). Par contre, un os au pied des Chevaux ponctués a donné $18400 \pm$ 350 BP (Lorblanchet 1995).

Plus encore que les dates, il nous semble que les techniques mises en œuvre au Pech-Merle ne doivent rien au hasard et sont investies d'une forte charge culturelle (et cultuelle) : il s'agit d'un art du pochoir, mobilisant bien que plus modérément qu'à Lascaux des aplats de peinture mixte (ici un mélange de manganèse et de charbon), pigments projetés au soufflé comme l'a montré Lorblanchet $(1980,1995)$.

D'un point de vue thématique, Pech-Merle se distingue par la présence de cercles échancrés (cf. Roucadour), de doigts repliés (cf. Gargas), d'un dessin d"Homme blessé" évoquant fortement ceux de Cougnac, de plus associé à un signe aviforme (ou de «type Le Placard »), de mégacéros (Ossuaire : Lorblanchet 1984a). Pour les empreintes de pouces, on notera, alors que le Pech-Merle est souvent le domaine du noir, qu'ils sont ici peints ou dessinés en rouge. Sur le Plafond des Hiéroglyphes ou le Panneau des Femmesbisons, des associations de figures féminines schématiques et de mammouths en tracé linéaire (Lorblanchet 1989), thème original s'il en est (Leroi-Gourhan 1965) évoquent indiscutablement Cussac (Aujoulat et al. 2004) ou plutôt l'in- 


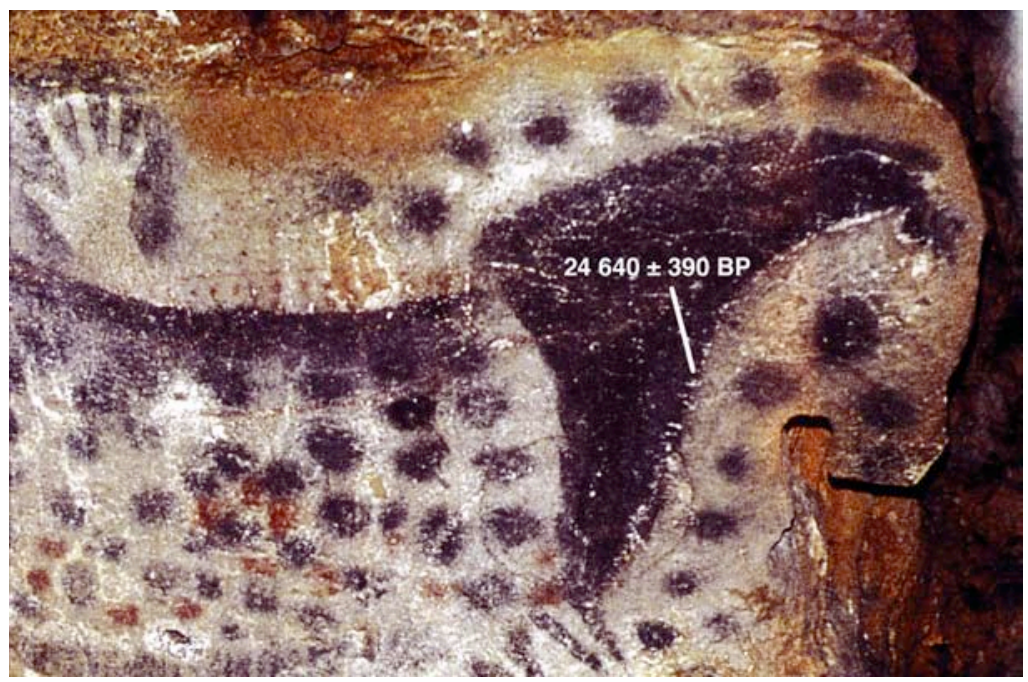

Figure 3 - Pech-Merle (Cabrerets, Lot). Cheval ponctué de droite réalisé au pochoir, cerné de ponctuations et empreintes de mains négatives noires. Les pigments de l'encolure ont fait l'objet d'une datation ${ }^{14} \mathrm{C}$ à $24640 \pm 390 \mathrm{BP}$ (C) SRA Midi-Pyrénées et Lorblanchet 1995).

Figure 3 - Pech-Merle cave (Cabrerets, Lot). Stencil doted horse on the right surrounded by black dots and black handprints. The pigments of the breast have been dated to $24,640 \pm 390 \mathrm{BP}$ (C) SRA Midi-Pyrénées et Lorblanchet 1995).

verse si l'on suit l'historiographie. Quant aux conventions, que ce soit sur le Panneau de la Cage au lion ou au Combel, le panneau dit des Antilopes, les animaux figurés, notamment les chevaux qui évoquent les chevaux ponctués sont aussi à têtes microcéphales, à corps ballonnés, pattes inachevées, le tout caractérisé par la rareté des détails anatomiques. Dans la catégorie des signes, les ponctuations réalisées au soufflé, en cercle (Femmes-bisons), en ligne, formant motif (Combel), associées ou non à des animaux (Chevaux ponctués, Cage au lion) ainsi que les empreintes de mains négatives caractérisent le dispositif du Pech-Merle que l'on a de plus en plus tendance à attribuer dans son ensemble au Gravettien (Lorblanchet 2004).

\section{Cougnac (Quercy)}

Autre passage obligé de l'art paléolithique quercinois, la grotte de Cougnac près de Gourdon (Lot) qui a fait l'objet des premières dates radionumériques obtenues sur pigment dans une grotte ornée paléolithique (Lorblanchet et al. 1990 ; Valladas et al. 1993). La Grande paroi et son fameux couple de mégacéros a livré les résultats les plus intéressants (fig. 4) : des échantillons prélevés sur le contour dessiné du premier cervidé a donné deux dates : $23610 \pm 350 \mathrm{BP}$ et $22750 \pm 390 \mathrm{BP}$, un échantillon issu de la bosse de la femelle indique $25120 \pm 390 \mathrm{BP}$, tandis qu'une quatrième date a donné $19300 \pm 270 \mathrm{BP}$ pour un prélèvement du dessin noir de sa croupe. Des datations nettement plus récentes attribuées à des passages au Magdalénien moyen ont donné pour le pigment charbonneux d'une ponctuation noire $14290 \pm 180 \mathrm{BP}$ et un métacarpien de renne avec stries de découpe est daté de $15000 \pm 200$ BP. Un débat s'en est suivi avec les partisans d'une fréquentation longue et récurrente du sanctuaire, argumentée par des indices de reprises de dessins, des rajouts (Lorblanchet 1994, 1995) et ceux qui, tablant sur l'unicité du dispositif, nuancent l'étalement des dates ${ }^{14} \mathrm{C}$ en interprétant cette dispersion due à des paramètres physico-chimiques ou taphonomiques (Clottes 1995 ; Sauvet 2004). Les unités graphiques de Cougnac paraissent en effet d'une homogénéité patente.

La thématique et le bestiaire renforcent l'association PechMerle - Cougnac : thème de l' « Homme blessé », omni-

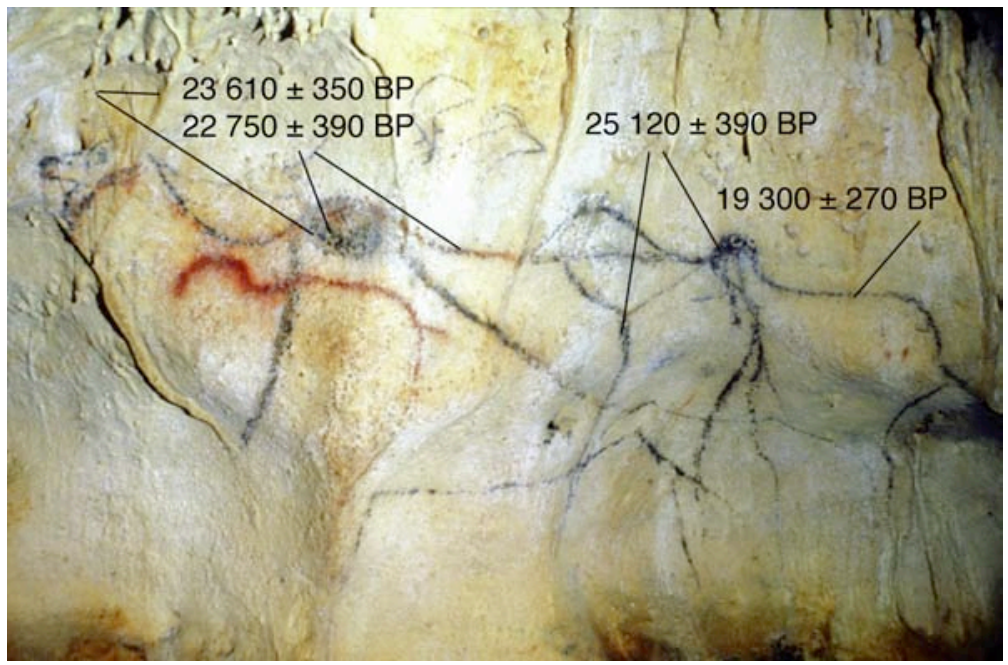

Figure 4 - Cougnac (Payrignac, Lot). Couple de mégacéros ayant fait l'objet de datations ${ }^{14} \mathrm{C}$ (๑ SRA Midi-Pyrénées d'apr. Lorblanchet et al. 1990, Lorblanchet 1995).

Figure 4 - Cougnac cave (Payrignac, Lot). Couple of Giant deer (Megaloceros) dated by ${ }^{14} \mathrm{C}$ method (C SRA Midi-Pyrénées according to Lorblanchet et al. 1990, Lorblanchet 1995). 


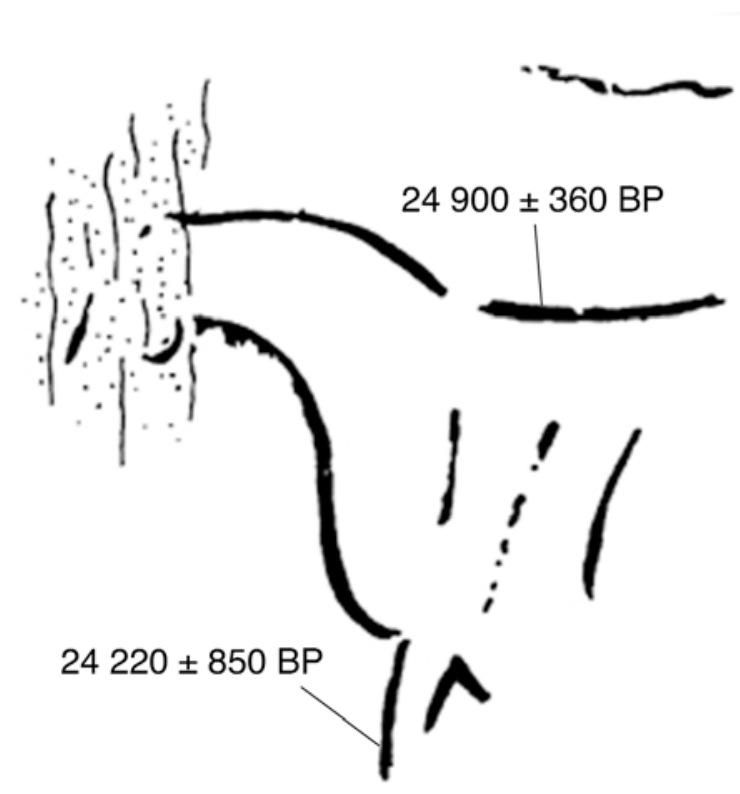

présence des représentations de mammouths, de mégacéros, du bouquetin, animaux silhouettés, aux détails corporels inexistants ou rarissimes, multiplication des signes aviformes / Le Placard, empreintes digitales, etc. et, comme désormais plusieurs autres sanctuaires ornés récemment datés, tout semble aller dans le sens d'une attribution de l'ensemble de Cougnac au seul Gravettien (Sauvet 2004).

\section{Mayenne-Sciences (Maine)}

Cette cavité ornée de dessins silhouettés dominés par les chevaux (majoritaires) et des mammouths noirs, réputée avec les grottes ornées d'Arcy pour être l'une des plus septentrionales, a fait l'objet de travaux récents qui ont permis d'en préciser et d'en confirmer le statut (Pigeaud 2001, 2004, 2005). Le corpus graphique n'est pas abondant mais typique d'un art que R. Pigeaud qualifie, avec une prudence extrême d' " anté-magdalénien », tout en excluant l'Aurignacien. Comme la plupart des sites évoqués dans ces pages, ses représentations animales ont souvent été citées comme des exemples d'un art dépouillé, sans détails anatomiques internes, des crinières en cimier, des têtes bec de canard, des perspectives semitordues, etc. S'y ajoutent des données concordantes : empreintes digitales rouges, datation d'un niveau à ossements brûlés provenant d'un sondage dans l'entrée (22 $600 \pm 380$ BP par la méthode classique ${ }^{4}$ ) et surtout les deux âges ${ }^{14} \mathrm{C}$ obtenus récemment par $\mathrm{H}$. Valladas à partir de pigments charbonneux pariétaux extraits des dessins noirs (fig. 5) : $24220 \pm 850$ et $24900 \pm 360$ BP confirmant l'âge supposé gravettien de ce sanctuaire (Pigeaud et al. 2003).
Figure 5 - Mayenne-Sciences (Thorignéen-Charnie, Mayenne). Dessin noir de cheval ayant fait l'objet de deux datations (relevé R. Pigeaud in Pigeaud et al. 2003)

Figure 5 - Mayenne-Sciences cave (Thorigné-en-Charnie, Mayenne). Drawing of a black horse dated by $\mathrm{C} 14$ method (tracing R. Pigeaud in Pigeaud et al. 2003).
II n'en reste pas moins que le dispositif graphique très probablement gravettien - Gravettien moyen proche des faciès à Noailles si l'on s'en tient aux datations pariétales de Mayenne-Sciences est inscrit dans une géographie nord-occidentale et atlantique qui n'est pas réputée pour ses indices d'occupation gravettienne ${ }^{5}$.

\section{2 - Dates "indirectes" : os fichés, sols archéolo- giques associés}

\section{Grotte de Gargas inférieure (Pyrénées)}

La grotte inférieure de Gargas (Hautes-Pyrénées) est réputée pour receler le plus grand nombre d'empreintes de mains négatives, la majorité aux doigts incomplets ou repliés, pas moins de 250 (Barrière 1979). Sur la Grande paroi des mains (fig. 6), des esquilles osseuses fichées en paroi ont été repérées en plusieurs endroits à proximité des empreintes rouges et noires et l'une d'entre elles a été datée par H. Valladas de $26860 \pm 460$ BP (Clottes et al. 1992). Cela ne date pas pour autant automatiquement l'âge de réalisation des empreintes, mais il est légitime de supposer qu'il y a de fortes présomptions pour établir l'association que l'on qualifiera de possible sinon hautement probable. Le point commun entre l'acte de ficher des esquilles osseuses dans des anfractuosités de la paroi et celui d'apposer des empreintes de mains aux doigts repliés est l'absence de référent animalier, et probablement artistique.

On trouve également, mais cette fois dans le Sanctuaire des mains, une frise d'empreintes de pouces repliés, de teinte noire, associée à d'autres empreintes de mains noires et rouges.

(4) En général, des reprises de datations par SMA (AMS) tendent à vieillir presque toujours les âges ${ }^{14} \mathrm{C}$ obtenus naguère par la méthode conventionnelle comme c'est le cas ici.

(5) Mais n'en est-il pas de même pour l'Ardèche de Chauvet aussi peu réputée pour un bilan aurignacien tout aussi lacunaire? 


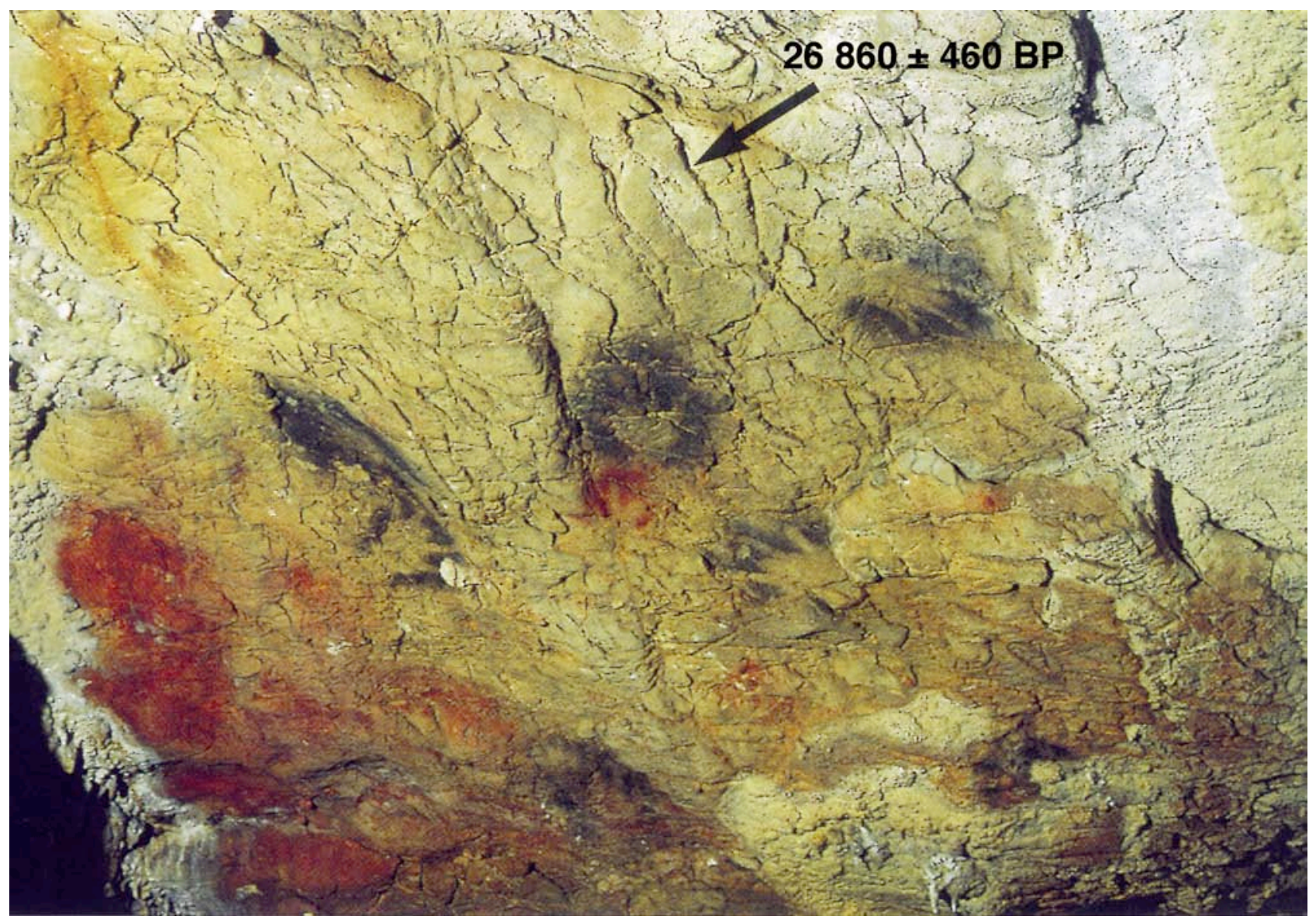

Figure 6 - Gargas inférieure (Aventignan, Hautes-Pyrénées). Grande paroi des mains et os fiché daté de $26860 \pm 460$ (@) Clottes in Clottes et Courtin 1994).

Figure 6 - Gargas, lower cave (Aventignan, Hautes-Pyrénées). Handprint stencils on the Great wall with bedded bone dated to $26860 \pm 460$ (@) Clottes in Clottes et Courtin 1994).

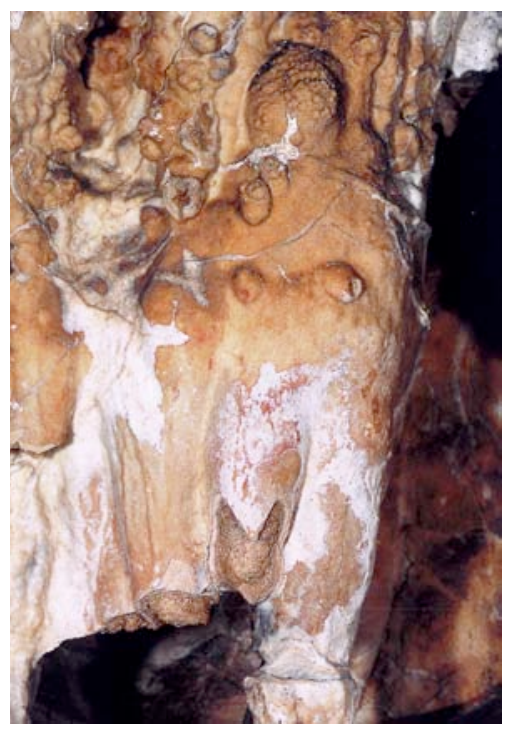

Figure 7 - Grande Grotte d'Arcy (Arcy-sur-Cure, Yonne). Relief naturel évoquant une représentation féminine portant des traces d'ocre rouge sur la cuisse et la poitrine (C) Baffier et Girard 1998).

Figure 7 - Arcy Large Cave (Arcy-sur-Cure, Yonne). Natural relief suggesting a feminine figure with red traces on thigh and breast (@ Baffier et Girard 1998). 


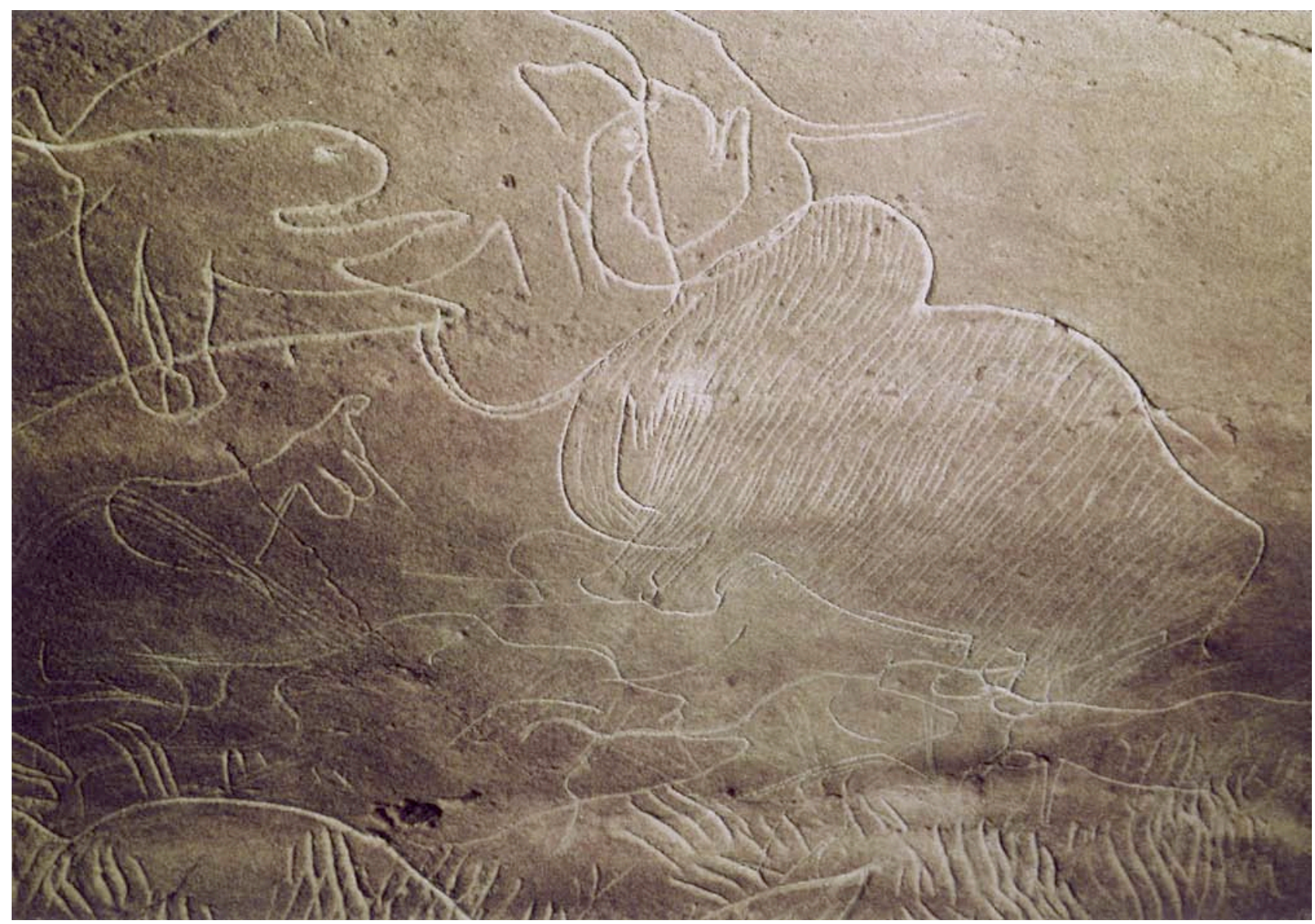

Figure 8 - Cussac (Le Buisson-de-Cadouin, Dordogne). L'un des principaux panneau de gravures monumentales avec, au centre, le thème mammouth / représentation féminine stylisée évoquant Pech-Merle (@Aujoulat et al. 2004 - CNP, Ministère de la Culture).

Figure 8 - Cussac cave (Le Buisson-de-Cadouin, Dordogne). One of the main panel with monumental engravings with, in the center, the Mammoth topic / stylized feminine figure reminiscent of Pech-Merle (CAujoulat et al. 2004 - CNP, Ministère de la Culture).

L'originalité de ce motif permet un rapprochement avec le thème analogue des pouces rouges du Pech-Merle (supra). La thématique et surtout le style du bestiaire, notamment pour le Sanctuaire des Gravures ont, depuis la fouille du porche, permis d'établir une comparaison entre art pariétal et art mobilier. En effet, plusieurs plaquettes ou galets portent des gravures au style comparable en tout point à celui des parois du Sanctuaire des gravures, que ce soit le Camarin, la Pierre Noire ou le Baldaquin (Breuil et Cheynier 1958 ; Barrière 1979) : art de la silhouette, profil absolu, style « fil de fer », etc. Les pièces d'art mobilier ont été découvertes dans un niveau de « Périgordien à burins de Noailles » (= Gravettien moyen) et c'est peut-être vers cette phase que s'orienterait, pour tout ou partie, le dispositif pariétal de Gargas (Breuil et Cheynier 1958).

Les conventions de représentations animales avec recours à la perspective semi-tordue - chère à l'abbé Breuil -, peu ou pas de détails anatomiques, des corps souvent dif- formes, la présence du mégacéros (Camarin)... sont autant d'éléments retenus pour un âge ancien et notamment par A. Leroi-Gourhan dans la définition de son style II. Claude Barrière y a reconnu une diachronie assez complexe avec pas moins de douze stades regroupés en trois phases commençant avec les tracés digitaux non figuratifs et s'achevant par l'expression en figuratif naturaliste et l'évocation du mouvement (Barrière 1976). II faut bien reconnaître que les graphismes animaliers de Gargas présentent en effet une palette assez variée qui rendent probablement plus complexes le recours par trop mécaniste et peu critique à une courte liste de conventions ${ }^{6}$.

\section{Grande Grotte d'Arcy (Bourgogne)}

L'opportunité de découvrir des sols archéologiques au pied de parois ornées, puis de dater des échantillons autorise l'hypothèse d'associations indirectes.

(6) Des travaux ont été récemment repris à l'initiative de $P$. Foucher qui devraient livrer des éléments nouveaux quant à la chronologie et la fréquentation de cet important sanctuaire pyrénéen pour lequel une nouvelle date a été obtenue sur bois de renne : $25050 \pm 170$ BP (Foucher 2004). 
C'est le cas pour la Grande Grotte d'Arcy (Yonne) avec un domaine de dates ${ }^{14} \mathrm{C}$ à partir de charbons découverts au sol et compris entre 28000 et 24500 BP (Baffier et Girard 1992, 1995b, 1998 ; Girard et al. 1996). Deux dates concordantes ont été obtenues sur un mouchage de torche sur la Frise rouge de la Salle des noyaux de cerise : $26470 \pm 390$ et $27080 \pm 400$ BP (Valladas et al. 2001) confirmant l'ancienneté du sanctuaire. Au pied d'une paroi ornée d'empreintes de mains négatives, ou plutôt au pied de la paroi opposée comprenant un aplat rouge, une empreinte de main aux doigts incomplets et les segments d'un grand herbivore, des os brûlés ont été datés de $24660 \pm 330$ et $26700 \pm 410$ BP (Baffier et Girard 1995b).

Le bestiaire confirme cette présomption d'une attribution de la Grande Grotte au Gravettien avec, notamment, l'abondance des mammouths et des conventions typiques des phases anciennes (Baffier et Girard 1998 p. 91-95). Comme à Gargas, Cosquer et Pech-Merle (Plafond des Hiéroglyphes), des tracés digitaux sont également attestés à Arcy, de même que des empreintes de mains positives (cf. Cosquer) et surtout négatives, entières ou aux doigts incomplets.

Un relief naturel de concrétions souligné d'ocre (fig. 7) évoque par ailleurs l'image d'une figure féminine stéatopyge (Baffier et Girard 1995a) comme l'art mobilier gravettien en a livré de si nombreux exemples.

\section{Grotte de Cussac (Périgord)}

Cette extraordinaire découverte de septembre 2000 a sérieusement enrichi le corpus des grottes ornées d'âge supposé gravettien, notamment pour le sud du Périgord (Aujoulat et al. 2001, 2004). Les arguments pour une attribution au Gravettien sont peu discutables : conventions avec des animaux en profil absolu, perspectives semi-tordues (cf. Sanctuaire des gravures de Gargas), présence répétée du thème «Femme - Mammouth » déjà relevé au Pech-Merle, profils féminins stylisés (fig. 8) extrêmement proches de plusieurs représentations du Plafond des Hiéroglyphes toujours au Pech-Merle, etc.

Mais plus exceptionnelle encore est la présence de restes humains concentrés dans plusieurs bauges et qui pourraient bien correspondre à des dépôts volontaires de corps en contexte karstique orné. Au nombre de trois, peut-être quatre, et s'il s'agit bien de sépultures, une attribution au Gravettien n'aurait rien d'étonnant tellement cette période est réputée pour ses exemples de sépultures individuelles, doubles, voire multiples (Henry-Gambier ce volume), en France (Cro-Magnon, Abri Pataud) mais surtout en Italie et en Europe centrale (Dolní Vestonice, Pavlov, Brno...). Moins clairement intégrée à la sphère gravettienne s.s., les sépultures de Soungir (Russie) sont également contemporaines de Cussac.
Ces éléments convergeant pour une fréquentation de Cussac par les Gravettiens ont été logiquement confirmés par l'obtention d'une date à partir d'un échantillon osseux humain - le seul dont le collagène était suffisamment bien conservé - de la sépulture du locus $1: 25120 \pm 120$ BP (Aujoulat et al. 2004) ${ }^{7}$.

\section{3 - Le cas particulier de Chauvet}

\section{Grotte Chauvet-Pont d'Arc (Ardèche)}

Ne revenons pas sur l'attribution d'ensemble à une phase de l'Aurignacien, certes plus récente que l'Aurignacien ancien. Ceci rappelé, dès la publication des premiers résultats de datations « directes » de prélèvements pariétaux (Clottes et al. 1995), l'obtention d'âges plus récents permet aux auteurs d'envisager, a minima, un passage des Gravettiens. En effet, à défaut d'œuvres pariétales, des dates de mouchages de torche et de charbons au sol cadrent avec une fréquentation de Chauvet postérieure à la phase d'ornementation centrée sur l'Aurignacien. Pour s'en tenir aux seules dates obtenues d'après l'analyse des charbons en paroi (mouchages) ou au sol (charbons, éléments de foyers), les échantillons datés se rencontrent depuis la Salle des Panneaux rouges, la Salle du Cierge, la Salle Hilaire, les galeries des Croisillons, des Mégacéros jusque dans la Salle du Fond. Pas moins de 14 dates s'échelonnent entre $27130 \pm 490$ et $22800 \pm$ 400 BP (Valladas et al. 2001, 2004, 2005). Le fait que toutes les dates " gravettiennes " relèvent soit de mouchages de torche, soit de charbons au sol plaide en faveur de l'hypothèse d'une seule génération de représentations pariétales datables.

D'ailleurs, et jusqu'à présent, l'équipe qui travaille à Chauvet exclut la présence d'éléments graphiques pariétaux autres qu'aurignaciens (Feruglio et Baffier $2005-\mathrm{p}$. 157), si ce ne sont les mouchages attribuables au Gravettien. Au contraire d'E. Guy qui relève des similitudes et conventions graphiques communes entre le tracé gravé d'une tête de cheval de la Salle Hillaire recouvrant des dessins aurignaciens et ce qu'il observe sur des représentations ornées gravetto-solutréennes du Parpalló, de La Pileta, Mayenne-Sciences, Pair-non-Pair, Foz Coâ, etc. (Guy 2004). Dans la galerie des Panneaux Rouges, il est vrai qu'un ensemble d'empreintes de mains négatives rouges associé à des ponctuations réalisées elles aussi au soufflé oblitère un tracé noir de mammouth (Aujoulat et al. 2001) et ne déparerait pas en contexte gravettien (fig. 9).

(7) Ce site, extrêmement prometteur, n'a été l'objet, pour l'heure, que des premiers inventaires et repérages et des travaux programmés auront lieu dès que les mesures conservatoires, l'équipement des sols et les problèmes d'acquisition foncière seront compatibles avec une entreprise pluridisciplinaire ambitieuse. 


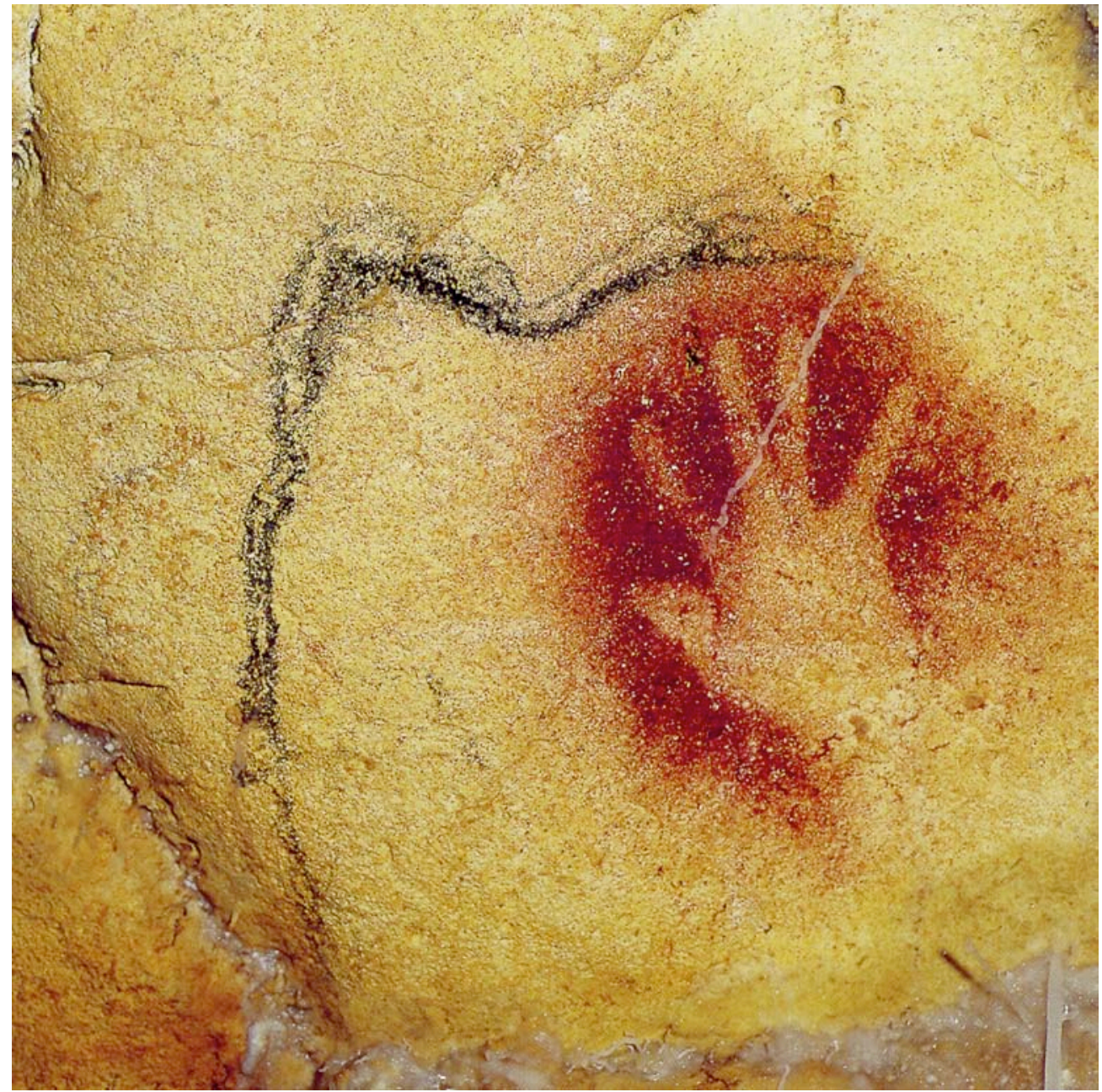

Figure 9 - ChauvetPont d'Arc NallonPont-d'Arc, Ardèche). Empreinte de main négative rouge et silhouette incomplète de mammouth noir du Panneau des mains négatives (@) Aujoulat in Clottes dir. 2001).

Figure 9 - ChauvetPont d'Arc cave VallonPont-d'Arc, Ardèche). Red stencil handprint and incomplete profile of a black mammoth in the Stencil handprint panel (C) Aujoulat in Clottes dir. 2001).

\section{2 - SANCTUAIRES ORNES GRAVETTIENS TRÈS PROBABLES, DATÉS PAR STRATIGRAPHIE}

\section{1 - Paroi ornée scellée par des dépôts archéolo- giques}

Nous entendons ici des parois ornées découvertes à l'occasion de fouilles ou travaux qui, en progressant, ont découvert ou redécouvert des œuvres jusqu'alors oblitérées par les sédiments, archéologiques ou non. Si l'un des exemples les plus fameux est Pair-non-Pair, citons la découverte plus récente de parois ornées à la grotte du Placard par L. Duport, travaux ensuite menés sous la conduite de J. Clottes et collaborateurs. Ils se rapportent à des éléments de parois ornés attribués au Solutréen (Clottes et al. 1990, 1991).

\section{Grotte de Pair-non-Pair (Gironde)}

II est de notoriété publique que cette cavité girondine située en rive droite de la Dordogne juste avant sa confluence avec la Garonne, certes modeste par son développement karstique n'en était pas moins un formidable gisement archéologique, exploré très tôt par
F. Daleau. D'après les différentes études tentant de reconstituer l'archéoséquence a posteriori (p. ex. Cheynier et al. 1963 ; Lenoir 1983 ; Roussot 1984b ; Delluc et Delluc 1991), celle-ci comprendrait a minima, un ou des niveaux attribués au Moustérien (Quina, MTA), au Châtelperronien, à l'Aurignacien ancien et récent, enfin au Gravettien (à gravettes, fléchettes, Font-Robert - sans Noailles - donc plutôt Gravettien ancien-moyen) ; sans compter des niveaux plus ou moins stériles ou paléontologiques, à carnivores (Hyène) probablement à rapporter à l'interpléniglaciaire du stade isotopique 3.

L'identification des parois gravées par F. Daleau à la fin du XIX siècle (de 1883 à 1898 pour l'essentiel) en fait l'une des doyennes de l'art franco-cantabrique. La partie basse des gravures est située juste au-dessus d'une ligne de foyers de la zone moyenne de la couche 3, soit le Gravettien (Cheynier et al. 1963) et les premiers traits gravés aperçus en 1883 étaient bien à la hauteur des niveaux gravettiens les plus récents (couches 6-7), donc oblitérés par la partie sommitale de la séquence. Des traces de peintures ont également été relevées.

Les différents auteurs ont noté une technique de gravure profonde, le recours au piquetage, des conventions gra- 


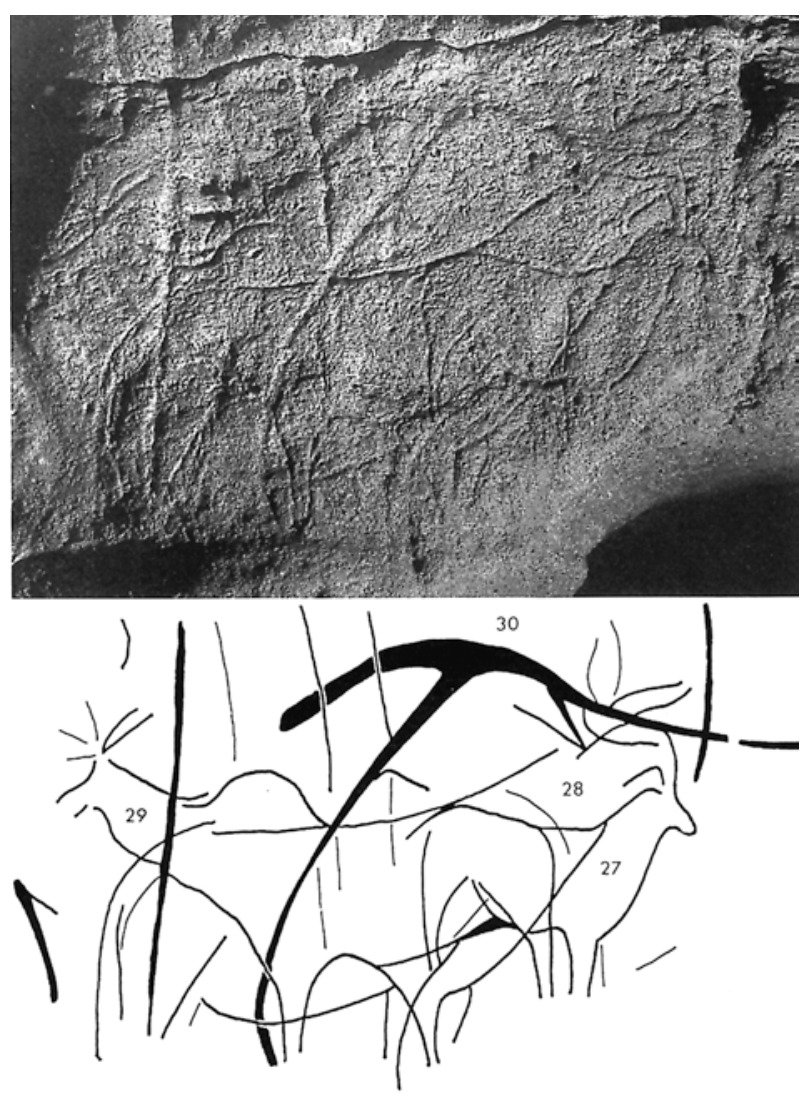

Figure 10 - Pair-non-Pair (Marcamps, Gironde). Panneau de gravures incluant un bouquetin (27), un cerf (28) et un mégacéros (29).(@ Delluc et Delluc 1991).

Figure 10 - Pair-non-Pair cave (Marcamps, Gironde). Engraved panel with ibex (27), red deer (28) and giant deer (29) (@ Delluc et Delluc 1991).

phiques des contours d'herbivores en profil strict, l'absence de détails anatomiques, des crinières de chevaux en cimier, ligne ventrale des mammouths en arche ou ogive, des encornures en perspective frontale, des têtes effilées (fig. 10). Notons par ailleurs la présence, dans le bestiaire, d'espèces réputées uniquement présentes avant le DMG comme le Mégacéros, ou majoritaires durant les phases anciennes comme le Mammouth ${ }^{8}$.

Concernant l'âge des panneaux de gravures qui totalisent une soixantaine d'unités graphiques (Breuil in Cheynier et al. 1963 ; Delluc et Delluc 1991), tous les auteurs s'accordent pour une attribution antérieure au Solutréen ${ }^{~}$. Les œuvres sont caractéristiques du style II de Leroi-Gourhan (1965), attribuées au cycle « aurignaco-périgordien » de Breuil (1952). On résumera en indiquant que l'ensemble gravé de Pair-non-Pair est obligatoirement antérieur au Solutréen, voire au Gravettien récent, donc plus probablement d'âge Gravettien (Roussot 1984b ; Delluc et Delluc 1991) ancien-moyen ou relevant d'une période antérieure.

\section{Grotte d'Oreille d'Enfer (Périgord)}

On connaît bien l'histoire de cet abri du vallon de Gorge d'Enfer (Guichard et al. 1984) et la découverte d'un ensemble de gravures sur une banquette pariétale recouvert de dépôts archéologiques incluant, pour l'Abbé Breuil, du Périgordien. Ce dernier précisant logiquement « ces figures enfouies sous un remplissage périgordien ne sauraient être plus récentes »(Breuil 1952 - p. 307). Donc Gravettien ou plus ancien. L'ensemble principal, profondément gravé, déposé depuis au Musée national de Préhistoire, montre des silhouettes peu suggestives d'herbivores (cervidé ? capridé ?), cervico-dorsales voisinant avec des séries de cupules évoquant plutôt les ensembles des sites aurignaciens concentrés autour des Eyzies.

\section{2 - Éléments pariétaux ornés (peints, gravés) découverts en stratigraphie}

La découverte d'éléments de parois ornés dans une unité stratigraphique (US) rapportée au Gravettien ne leur assure pas pour autant un âge contemporain, mais un terminus a quo ${ }^{10}$ ou ad quem ${ }^{11}$ suivant l'ordre diachronique que l'on adopte. C'est donc dans l'intervalle de temps incertain entre le terminus a quo et ad quem que se situera souvent l'événement recherché en question. D'un point de vue archéo-stratigraphique, de tels fragments ornés sont donc à rapporter soit à ladite période, soit à une période antérieure - qui ne peut souvent être que l'Aurignacien. L'âge de leur enfouissement - qui n'est pas automatiquement celui de leur chute -, a fortiori la date calendaire de la réalisation des UG peut donc différer sensiblement de celui de leur réalisation.

\section{Abri Labattut (Périgord)}

L'Abri Labattut à Sergeac (Dordogne) a livré des fragments de paroi ornée et des blocs gravés, dont plusieurs ont été découverts en stratigraphie par M. Castanet pour le compte de L. Didon entre deux niveaux de Gravettien, faces ornées contre terre (Delluc 1985 cité in Delluc et Delluc 1991). II y avait en fait au moins trois niveaux de Gravettien à Labattut (« Périgordien IV », « V » et « évolué »: Delluc et Delluc 1991) et D. de Sonneville-Bordes (1960) précise qu'il s'agit, pour le principal, d'un Périgordien à burins de Noailles. C'est visiblement des niveaux à Noailles que proviennent les pierres conservant des restes de peinture noire ou ocre, à anneaux et traits piquetés. Le bloc au cheval

(8) Malgré l'extraordinaire exception que constitue Rouffignac.

(9) Seule une feuille de laurier, aujourd'hui disparue, aurait été recueillie au sommet du remplissage.

(10) Limite à partir de laquelle.

(11) Limite jusqu'à laquelle. 


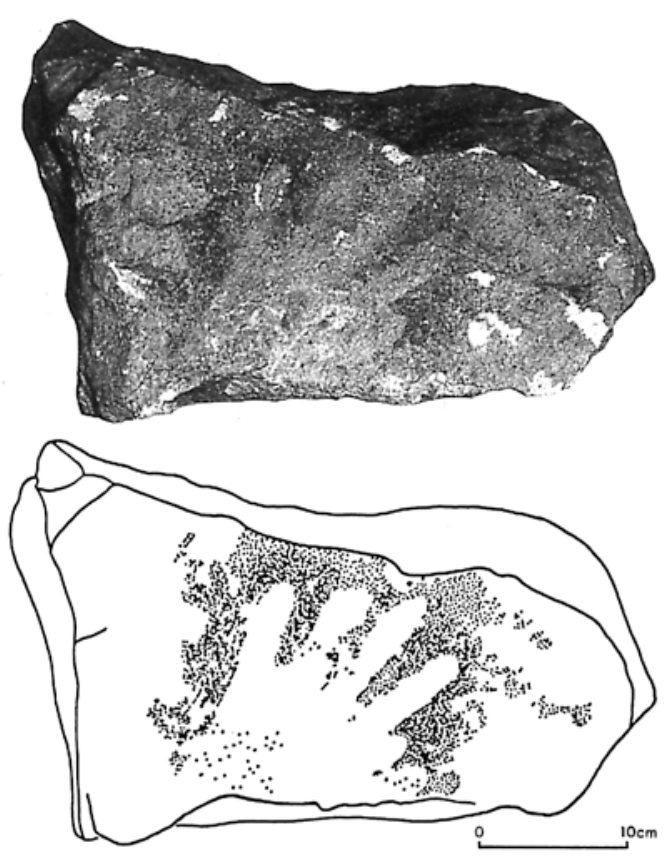

Figure 11 - Abri Labattut (Sergeac, Dordogne). Bloc de calcaire détaché de la paroi portant une empreinte de main négative noire (@ Delluc et Delluc 1991).

Figure 11 - Labattut rock shelter (Sergeac, Dordogne). Calcareous block broken off the wall with a black stencil handprint (@ Delluc et Delluc 1991).

gravé proviendrait, quant à lui, du niveau gravettien le plus ancien (Delluc 1991). Comme à Pataud (infra), des écailles de calcaire peintes proviennent aussi du niveau le plus récent du Gravettien ce qui indique un terminus ad quem.

Les trois œuvres majeures sont un cheval gravé à tête déformée comme prolongée par un museau, son ventre ballonné et des pattes inachevées (Delluc et Delluc 1991) conservé à New-York ; de grands fragments de paroi peinte $(3 \mathrm{~m})$ avec les restes de bichromie, un possible bison, un mammouth et un cerf à belle ramure, relevés par A. Glory et conservés au MAN ; enfin, la fameuse empreinte de main négative noire retrouvée en 1982 dans les réserves du MAN (fig. 11) est la seule découverte en contexte stratigraphique certain (Delluc et Delluc 1991).

De nombreux auteurs ont déjà mis à contribution Labattut qui a fait l'objet de multiples études comparatives, notamment avec Lascaux ${ }^{12}$. Des parallèles doivent immanquablement être établis avec nombre d'abris de la Vézère qui devaient être peints dès l'Aurignacien pour certains, mais surtout au Gravettien. Les conventions des représenta-

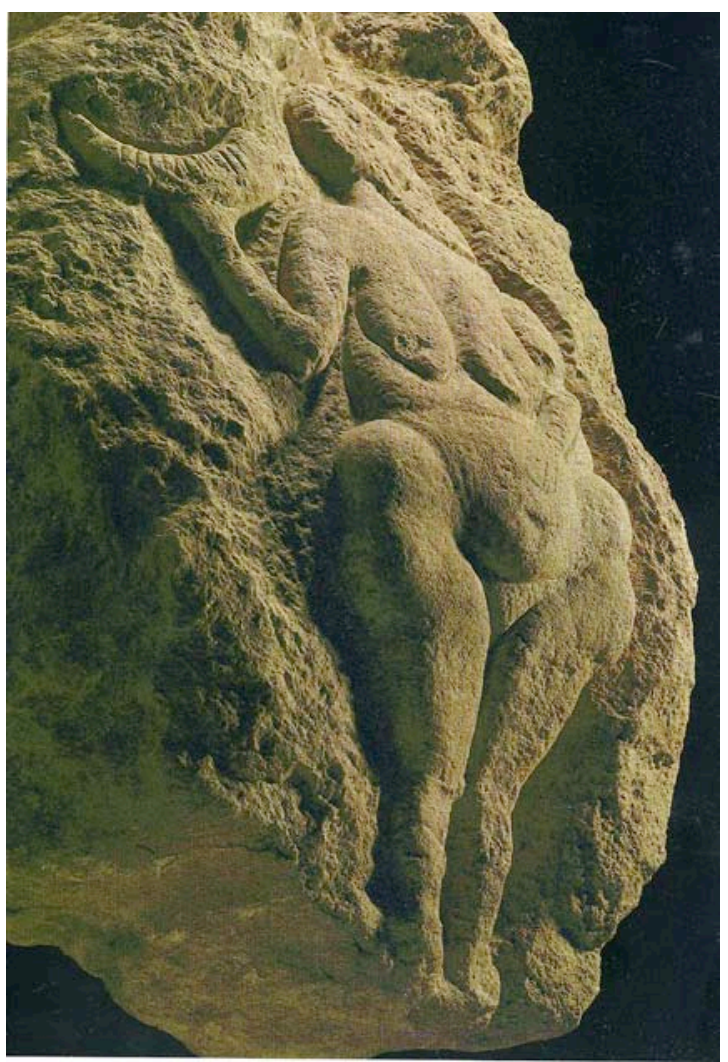

Figure 12 - Laussel (Marquay, Dordogne). Vénus à la corne vue de profil afin d'accentuer la parenté avec les statuettes d'art mobilier (@ Roussot 2000).

Figure 12 - Laussel rock shelter (Marquay, Dordogne). 'Venus' with horn. The picture shows the section view in order to accentuate the comparison with mobile art figurines (C Roussot 2000).

tions animales (perspective "tordue" d'une ramure de cerf, ventre ballonné d'un équidé, tête difforme à museau allongé, absence de détails anatomiques...) sont typiques des phases anciennes : style II de Leroi-Gourhan. L'empreinte de main négative est un élément qui renforce la parenté avec le monde gravettien.

\section{Grand Abri de Laussel (Périgord)}

Rendu célèbre par les fouilles du Dr. Lalanne et ses découvertes d'un art gravé et sculpté certes mobilier, mais aussi immobilier ${ }^{13}$ avant la Première Guerre mondiale, le Grand Abri de Laussel est un site-clef pour la connaissance de l'art gravettien. Le plus célèbre des blocs sculptés d'où a été extraite la fameuse Vénus à la corne a été découvert comme le montre une photo de 1911 dans un niveau sup-

(12) Pour un historique et une description détaillée : cf. Delluc et Delluc 1991, p. 151-166.

(13) Dans la notice de l'Atlas des grottes ornées, $A$. Roussot ne retient comme art pariétal, que la Vénus à la corne (Roussot 1984). 
posé de Gravettien moyen à Noailles (Roussot 1984a, 2000). II s'agit bien d'un art sur bloc à part entière car le support d'origine duquel a été extrait le célèbre bas-relief possédait des dimensions sans commune mesure avec le fragment actuel scié et aujourd'hui présenté au musée d'Aquitaine à Bordeaux.

Outre la Vénus à la corne, on compte d'autres blocs sculptés ou peints dont nous ne retiendrons ici que les plus importants : la Vénus à la tête quadrillée, la Carte à jouer (dont la provenance stratigraphique a été un temps controversée), la Vénus de Berlin et le Chasseur. Nous renvoyons à Delluc et Delluc 1991 pour l'historique des découvertes, un inventaire plus précis, la description et l'étude de ces œuvres emblématiques.

Si la totalité des blocs ornés ne peut être attribuée avec certitude à l'un ou l'autre des niveaux attestés à Laussel (Moustérien, Châtelperronien, Aurignacien, Gravettien, Solutréen inférieur, récent : Delluc et Delluc 1978, 1991), l'âge des célèbres blocs, ornés de représentations féminines, est moins douteux (Roussot 2000). Non seulement tous les éléments convergent pour un âge très certainement gravettien, mais ces blocs sculptés établissent un lien indiscutable avec l'art mobilier (fig. 12) et les fameuses statuettes féminines (Delporte 1979 ; Roussot 2000). Même si B. et G. Delluc (1991) insistent sur ce qui démarque ces œuvres de l'art pariétal gravettien, ce que l'on ne peut nier, on y retrouve cependant un certain nombre de conventions communes, au moins avec le groupe des statuettes mobilières : schématisme, hypertrophie de certaines parties anatomiques (seins, abdomen), silhouette inscrite dans un losange (Leroi-Gourhan 1965), absence de traits du visage, négligence du figuré des extrémités... suffisamment décrites pour y revenir ici.

\section{Abri Pataud (Périgord)}

Même si nous avons en mémoire ce fameux bouquetin gravé au plafond de l'abri aujourd'hui protégé par le bâti du petit musée ${ }^{14}$, le nom de Pataud ne vient pas naturellement lorsque l'on aborde le chapitre de l'art pariétal ${ }^{15}$. Or, le nombre de fragments de paroi peinte (blanc, rouge), gravée, le nombre d'éléments de toutes natures attribuables au registre de l'art immobilier et, a fortiori, mobilier est tel à Pataud qu'il convient de le mentionner ici. Un travail universitaire a du reste permis de faire le point sur cette question (Nivelle 2003), prolongeant les premières études de B. et G. Delluc (1991 op. cit.) synthétisé récemment (Delluc et Delluc 2004).

Prenant le relais de Laugerie-Haute, la stratigraphie est l'une des plus dilatées et les plus complètes qui soit pour le Pléniglaciaire récent et la transition des stades isotopiques 3 et 2 , avec pas moins d'une quinzaine de niveaux

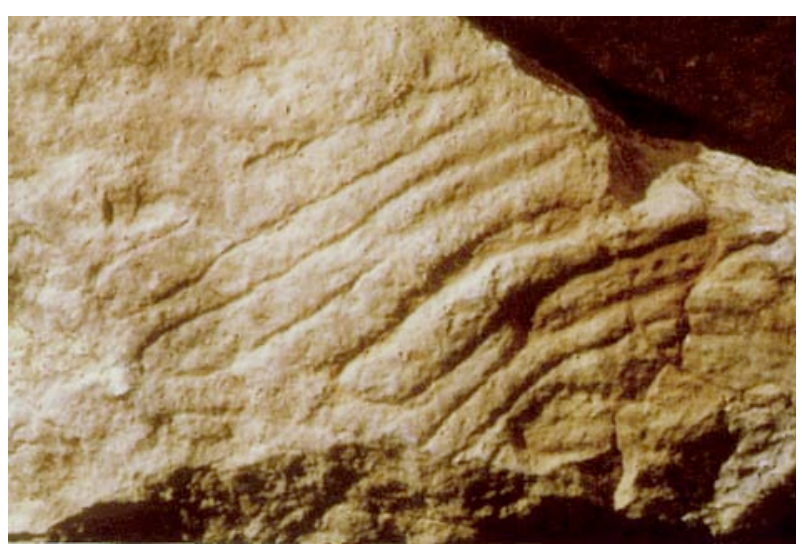

Figure 13 - Abri Pataud (Les Eyzies-de-Tayac, Dordogne). Gravettien moyen, bloc immobilier gravé de motifs serpentiformes et cupules (@ Delluc et Delluc 2004).

Figure 13 - Pataud rock shelter (Les Eyzies-de-Tayac, Dordogne). Middle Gravettian, engraved block with snake motif and cup marks (C Delluc et Delluc 2004).

d'occupations du Solutréen à l'Aurignacien ancien (Movius 1977 ; Bricker Dir. 1995). De plus, s'y ajoute une importante série de restes humains recueillis, pour l'essentiel, dans le Gravettien final («Périgordien VII » de D. de SonnevilleBordes).

À l'exception de la petite figure féminine stylisée, les blocs ornés découverts dans plusieurs niveaux gravettiens récent et final ne portent que des motifs non figuratifs ou difficiles de lecture: grandes cupules, lignes de cupules, ovales, motifs serpentiformes... (fig. 13). B. et G. Delluc ne retiennent que deux blocs ornés immeubles provenant de deux niveaux du Gravettien récent («Périgordien VI », niv. 3) ou final ("Périgordien VII » : niv. 2). Ce qui est quasi certain est que la paroi de l'abri lui-même devait être peinte ou gravée (archives Glory in Delluc et Delluc 1991 - p. 211). Le Gravettien final a fait l'objet de onze dates ${ }^{14} \mathrm{C}$ s'étageant entre $19300 \pm 170$ et $21940 \pm 250 \mathrm{BP}$ tandis que pour le Gravettien récent (ex-Périgordien $\mathrm{VI}$ ), les âges ${ }^{14} \mathrm{C}$ s'échelonnent entre $21540 \pm 160$ et $24440 \pm 740 \mathrm{BP}$ (Bricker et al. 1995 in Bricker dir. op. cit.).

\section{Abri Vignaud (Périgord)}

Voisin du précédent, donc sous l'abri au bouquetin gravé (musée Pataud), l'Abri Vignaud a livré un fragment de paroi peinte découvert dans un niveau de "Périgordien V" à Noailles (donc Gravettien moyen) à l'occasion d'un sondage conduit par S. Blanc en 1945 et retrouvé par B. et G. Delluc $(1987,1991,2004)$. Il s'agit d'une tête de cerf pein-

(14) Ce bâti correspond au petit musée de l'Abri Pataud, mais l'abri lui-même est un gisement distinct de Pataud s.s., en fait l'étage supérieur de la ligne d'abris dans le prolongement sagittal de l'abri Vignaud dénommé abri Movius (Delluc et Delluc 2004).

(15) Par exemple , il ne figure pas dans l'Atlas des grottes ornées. 


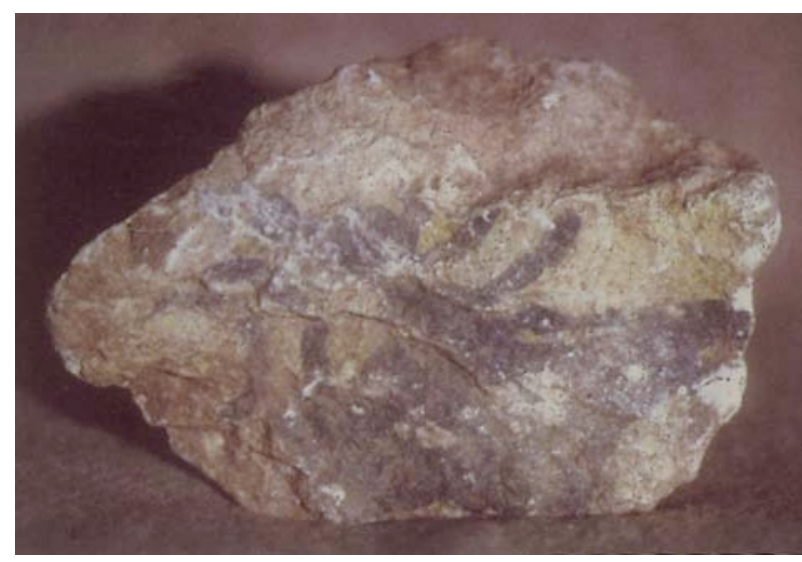

Figure 14 - Abri Vignaud (Les Eyzies-de-Tayac, Dordogne). Gravettien moyen à Noailles. Plaque de calcaire détachée de la voûte et portant une tête de cerf peinte en noire (C) Delluc et Delluc 2004).

Figure 14 - Vignaud rock shelter (Les Eyzies-de-Tayac, Dordogne). Middle Gravettian with Noailles. Slab of calcareous broken off the ceiling with a red deer head painted in black (@ Delluc et Delluc 2004).

te en aplat noir (fig. 14) et présentant des conventions évoquant certaines figures de Lascaux comme son museau effilé ou l'implantation nucale de l'oreille (Delluc et Delluc 1991). Cette plaque de calcaire $(16,5 \times 11 \times 6,5 \mathrm{~cm})$ est un indice précieux pour des rapprochements ou la définition d'un art gravettien et les auteurs insistent sur son caractère « très exceptionnel ", compte tenu des conventions rapprochant en effet ce figuré de certaines unités graphiques de Lascaux. Une fois encore, précisons que cette découverte stratigraphique ne constitue qu'un terminus a quo pour l'âge de la peinture : elle peut donc être soit d'âge
Gravettien moyen à Noailles, soit antérieure. D. Peyrony signale à Vignaud des niveaux de Moustérien et d'Aurignacien, mentions confirmées et complétées par une fouille de sauvetage beaucoup plus récente, inédite, pour laquelle J.-M. Geneste (1984) mentionne des niveaux moustériens, aurignaciens et gravettiens.

\section{3 - SANCTUAIRES ORNÉS GRAVETTIENS PRO- BABLES : ANALOGIES TECHNIQUES, THÉMATIQUES OU CONVENTIONNELLES FORTES}

\section{1 - Groupe du Quercy : Roucadour, Les Merveilles, Les Fieux, Marcenac, Frayssinet-le-Gélat (Lot)}

Nous commençons par le groupe quercinois car il nous semble le plus homogène, le plus typé, l'un des mieux documentés, mais aussi l'un des mieux connus grâce aux travaux de M. Lorblanchet qui ont favorisé une vision d'ensemble autorisant ce type de bilan.

\section{Grotte de Roucadour}

L'ensemble des unités graphiques de Roucadour (Thémines, Lot) découvertes en 1962 ont été relevées jadis par l'abbé Glory - peu de temps avant sa mort - (Glory 1965b), aidé de l'abbé Villeveygoux et des inventeurs (Coussy 2005) auxquels a succédé aujourd'hui l'équipe dirigée par M. Lorblanchet et J.-M. Le Tensorer. Elle s'insère très bien dans le premier groupe des sanctuaires quercinois (Lorblanchet 1972) rapprochée du style II/III de LeroiGourhan, lequel évoquait pour Roucadour une parenté avec Gargas dans la réédition de l'ouvrage " Préhistoire de l'art occidental » augmentée de 1971. Tous les éléments convergent en effet pour une attribution - pro parte pour le moins - à un art gravettien : cercles échancrés analogues à celui du panneau des chevaux ponctués du Pech-Merle,

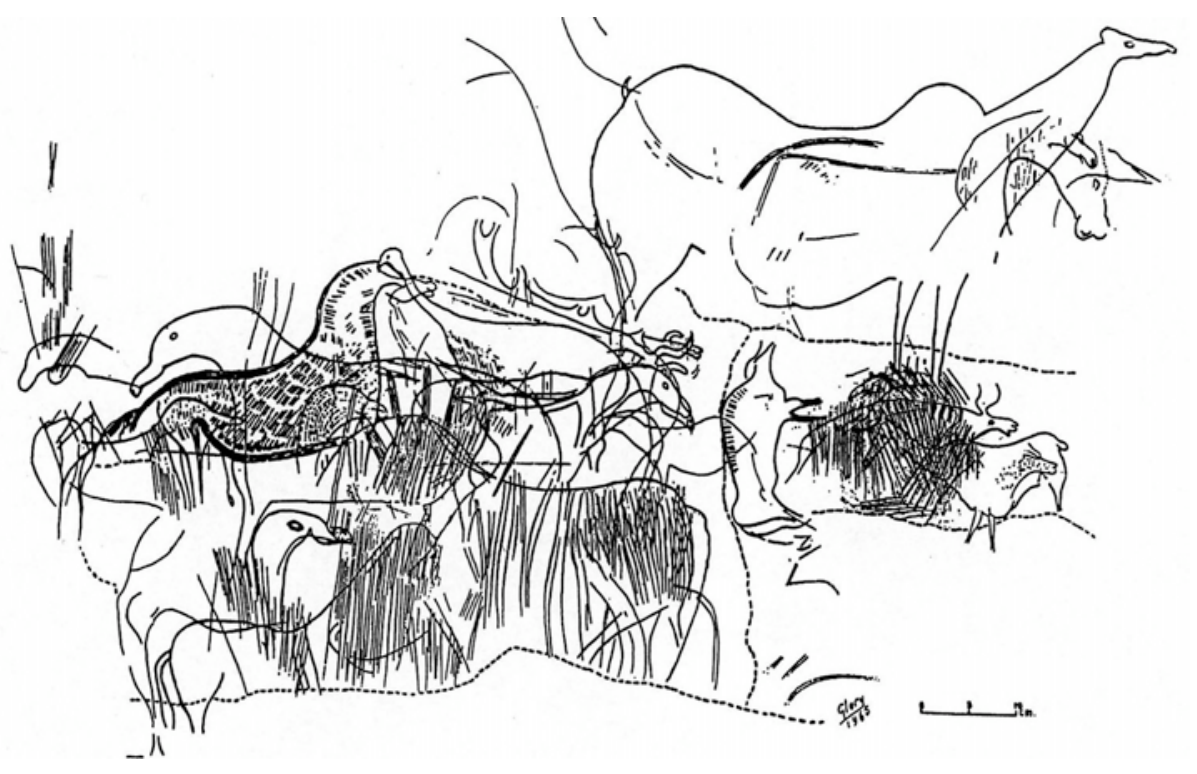

Figure 15 - Grotte de Roucadour (Thémines, Lot). Panneau avec plusieurs générations de raclages et gravures incluant des animaux aux corps difformes et museaux « becs de canard » (relevé A. Glory 1965 in Pigeaud 2005).

Figure 15 - Roucadour cave (Thémines, Lot). Panel with several generations of scrapings and engravings showing animals with distorted bodies and noses 'ducky beak' (tracing A. Glory 1965 in Pigeaud 2005). 


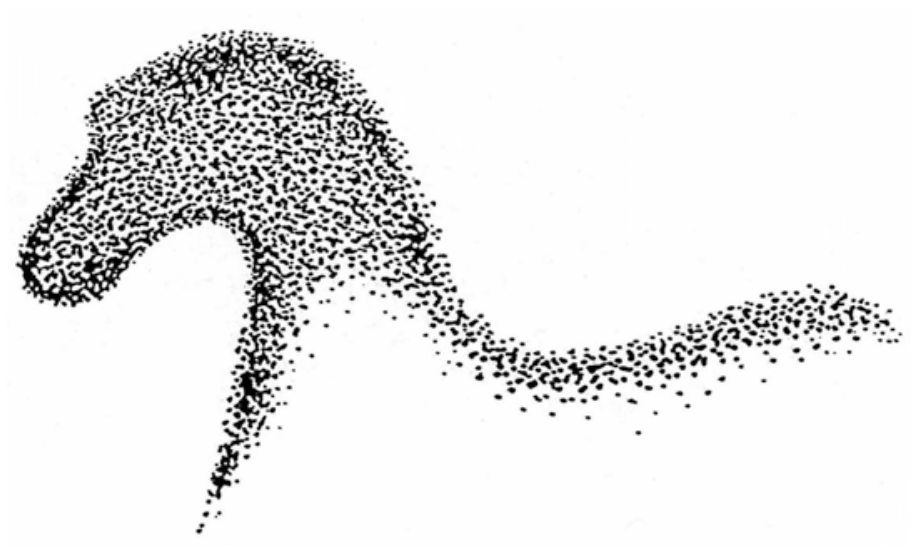

Figure 16 - Grotte des Merveilles (Rocamadour, Lot). Protomé de cheval réalisé au pochoir noir (relevé M. Lorblanchet in Lorblanchet, 1970.). Comparer avec Le Portel fig. 19.

Figure 16 - Les Merveilles cave (Rocamadour, Lot). Forehand of a black horse painted with stencil (tracing M. Lorblanchet in Lorblanchet, 1970.). To be compared with Le Portel fig. 19. surabondance des mégacéros, profil absolu des représentations d'herbivores, perspectives semi-tordues très proches de celles de Gargas, têtes nissocéphales à museau effilé ou tréflé, contours inachevés au niveau des membres, mammouths difformes à la ligne ventrale en arche, paire d'empreintes de mains négatives rouge et noire peintes apposées sur une paroi raclée, etc. (Lorblanchet 1984b ; 2004). De l'étude du panneau principal (fig. 15), l'abbé Glory $(1965 a, b)$ y voyait par contre un phasage incluant l'Aurignacien (empreintes de mains, chevaux " becs de canard ") et le Gravettien (cheval à robe ponctuée, bison, mégacéros, etc). Les travaux en cours enrichiront et préciseront à n'en pas douter cet art gravettien du Quercy.

\section{Grotte des Merveilles}

Ce petit ensemble du causse de Gramat à Rocamadour (Lot) émerge sans problème dans l'ensemble attribuable à la période ancienne de l'art du Quercy (Lorblanchet 1970) : peintures en aplat noir avec intervention du pochoir, contours d'animaux difformes et incomplets dépourvus de détails anatomiques, empreintes de mains négatives et grosses ponctuations rouges réalisées au soufflé (Lorblanchet 1970). L'un des protomés de chevaux évoque l'un de ses homologues bruns du Portel (fig. 16).

\section{Grotte des Fieux}

Au nord de Rocamadour, la grotte des Fieux à Miers (Lot) est également connue pour une longue séquence couvrant le Paléolithique moyen, l'Aurignacien, plusieurs niveaux de Gravettien sous le porche Est (couche F1c : $23900 \pm$ $330 \mathrm{BP}$ ), des indices de passages gravettiens à FontRobert sous le porche Ouest, un riche ensemble non daté attribuable à un Gravettien final (Guillermin, ce volume) avec quelques indices de passage solutréen achevant la séquence pléistocène (Champagne et Jaubert 1986 ; Champagne et al. 1990).

La zone ornée se limite à une petite salle comprenant 14 empreintes de mains négatives rouges et noires (fig. 17), des dizaines de ponctuations digitales rouges (Glory 1965), des esquilles osseuses fichées en paroi ${ }^{16}$ et un ensemble complexe de gravures conservées sur un mas-

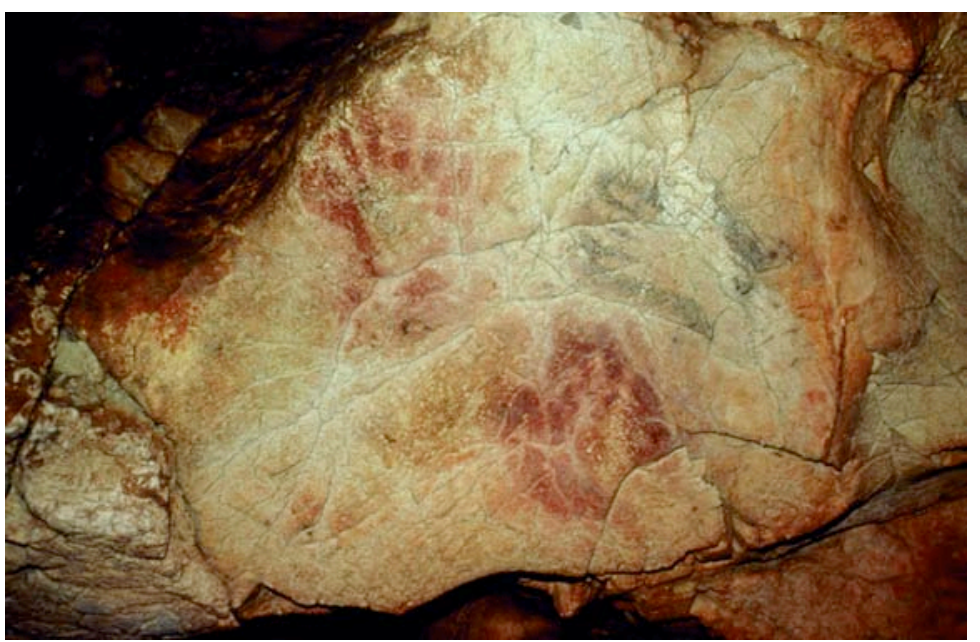

Figure 17 - Grotte des Fieux (Miers, Lot). Panneau principal des empreintes de mains négatives noires et rouges. (C J. Clottes SRA Midi-Pyrénées).

Figure 17 - Les Fieux cave (Miers, Lot). Main panel with black and red stencil handprints.

(C) J. Clottes SRA Midi-Pyrénées).

(16) Signalées par nous-même à M. Lorblanchet, il tenta d'en faire dater une sans succès, l'échantillon n'étant pas suffisamment riche en collagène. 


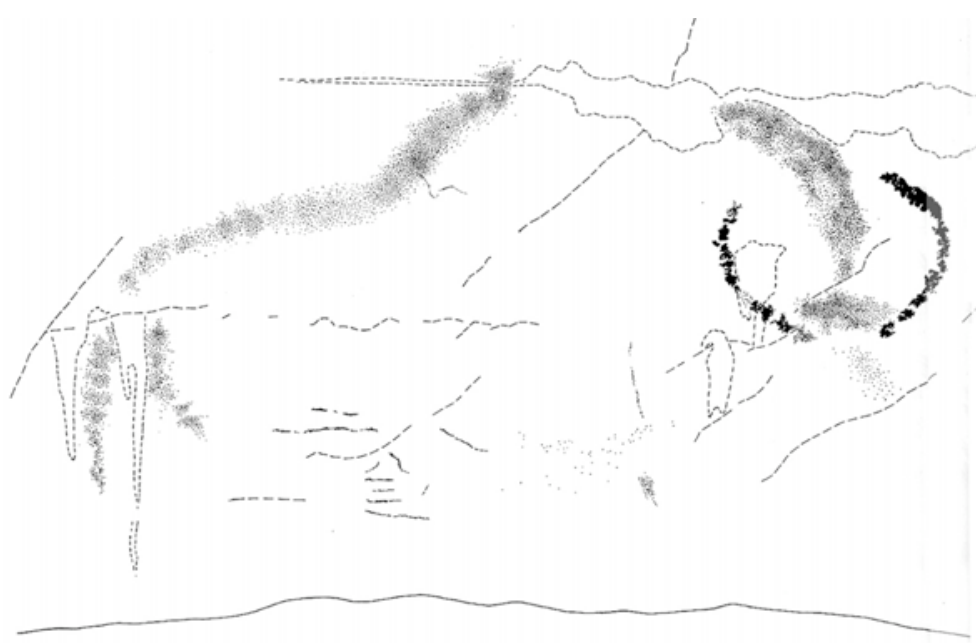

Figure 18 - Grotte de Marcenac (Cabrerets, Lot). Silhouette de bison bichrome réalisé par ponctuations juxtaposées au pochoir (relevé M. Lorblanchet in Lorblanchet 1984).

Figure 18 - Marcenac cave (Cabrerets, Lot). Profile of a bichromatic bison painted with a series of dots performed with stencil technique (tracing M. Lorblanchet in Lorblanchet 1984).

sif stalagmitique central : séries de cupules, avant-train de bouquetin et possible cheval ponctué figuré par cupules juxtaposées, gravures de mammouth, le massif stalagmitique évoquant lui-même le profil du proboscidien, restes de deux empreintes de mains rouges oblitérant le premier ensemble de gravures cupulées, etc. (Lorblanchet 1984c, 1989, 2005). Comme sa voisine Les Merveilles, il ne fait aucun doute que Les Fieux soit à associer au premier groupe des grottes du Quercy, attribution relative renforcée si l'on met en perspective l'archéoséquence des porches Ouest et Est qui s'achève par le comblement de limon soufflé (couche E, Gravettien final) contemporain du DMG, vers 22-20 ka BP. Avant ce dépôt, la lumière du jour devait pénétrer jusqu'à l'entrée de la salle ornée.

\section{Grotte de Marcenac}

Cette cavité voisine de Pech-Merle (Cabrerets, Lot) recèle quelques peintures et une paroi gravée étudiée en premier par l'abbé Lemozi (1959/1965). Y est remarquable la présence d'un grand bison au contour réalisé par une juxtaposition de gros points noirs apposés au soufflé et une magnifique perspective semi-tordue de l'encornure, peinte également par la technique de ponctuations juxtaposées (fig. 18) mais d'un noir différent de la silhouette générale (Lorblanchet 1984d, 1989). Quant aux gravures d'herbivores, notamment une belle frise de cerfs, un bouquetin, leurs silhouettes très conventionnelles avec des têtes trop petites, des museaux effilés, un signe quadrangulaire quadrillé de type blason rappellent plusieurs représentations de Lascaux ou de sanctuaires à gravures de style ancien proche du style III de Leroi-Gourhan. Autre rapprochement à mentionner avec Lascaux, une figure de bovidé bichrome, noir et rouge (Lorblanchet 1989). Comme Roucadour, Marcenac est importante pour la connaissance de l'art peint mais aussi gravé du Gravettien quercinois.

\section{Grottes de Frayssinet-le-Gélat}

Benjamines des cavités ornées du Quercy, les deux grottes de Combe Nègre à Frayssinet-le-Gélat (Lot),

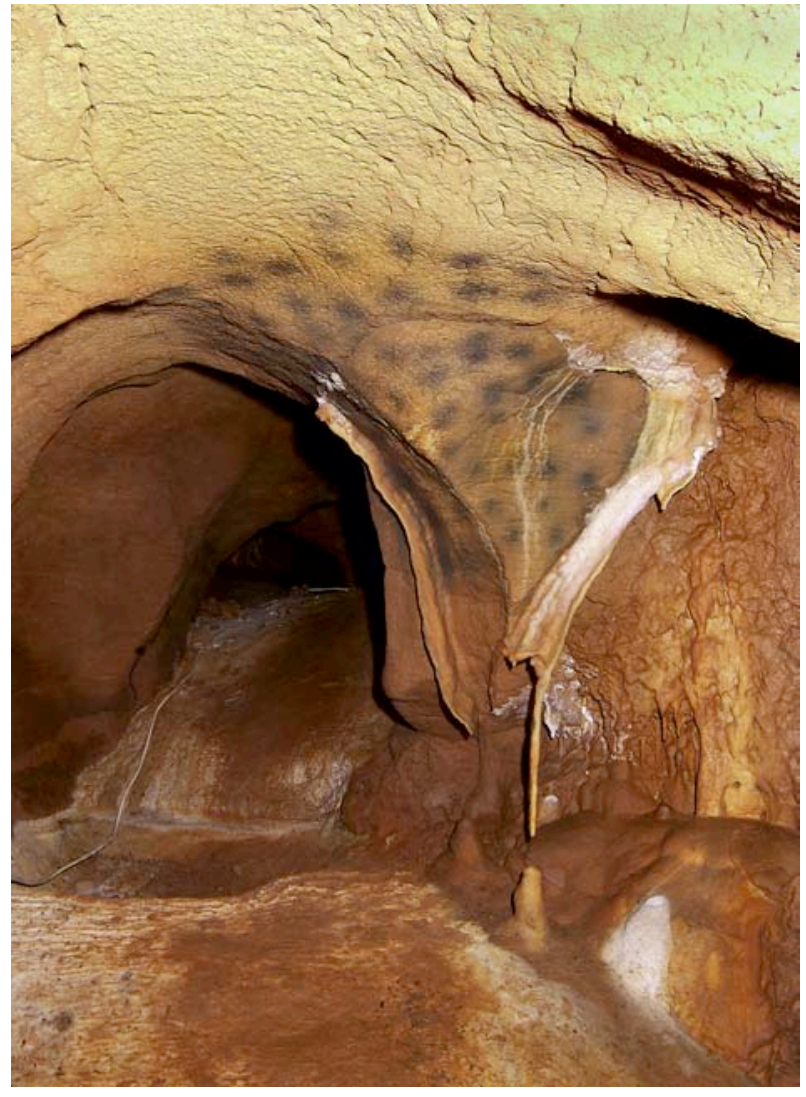

Figure 19 - grotte de Combe-Nègre 2 (Frayssinet-le-Gélat, Lot). Panneau de ponctuations noires de la première salle (C J.-F. Peiré, DRAC Midi-Pyrénées in Feruglio, Jaubert 2003.)

Figure 19 - Combe Nègre 2 cave (Frayssinet-le-Gélat, Lot). Panel of black dots in the first chamber (@ J.-F. Peiré, DRAC Midi-Pyrénées in Feruglio, Jaubert 2003). 
découvertes en 2001 par des spéléologues de Bouriane (Jaubert 2001) ont fait l'objet de relevés par $M$. Lorblanchet (Combe Nègre 1), V. Feruglio et moi-même (Combe Nègre 2), (Feruglio et al. 2005 ; Feruglio et al. 2007). Les deux galeries, aujourd'hui disjointes, appartiennent à un même ensemble typique de ce premier groupe des cavités ornées quercinoises. Combe Nègre 1 comprend le dessin d'une empreinte de petite main noire, des ponctuations digitales noires, un petit herbivore (bison ?) et un couple de chevaux dessinés au doigt et présentant des conventions analogues aux équidés du Pech-Merle. Pour la seconde galerie, c'est une impressionnante série de ponctuations réalisées au soufflé pas moins de 460 ! - isolées, regroupées par panneaux formant grille, couvrant voûte (fig. 19) ou pendentif (noires) ou encore disposées par registre en long bandeaux horizontaux (noir dominant interrompu de rouge) marquant l'entrée et la fin du sanctuaire. Des impacts dans l'argile calcitée et le frottis de doigts enduits de noir sont également à retenir. Des datations ${ }^{14} \mathrm{C}$ et TIMS sont en cours indiquant que le dernier voile de calcite est postérieur au DMG ${ }^{17}$.

\section{Roc de Vézac (Dordogne), Moulin de Laguenay (Corrèze)}

Cette cavité de la rive droite de la Dordogne a été rapprochée du groupe quercinois évoqué supra puisque tous les éléments graphiques l'y conduisent : ponctuations, couple d'empreintes de mains négatives rouge et noire, signes réniformes évoquant les cercles échancrés de Roucadour ou du Pech-Merle mais ici en bas-relief (Aujoulat 1984b).

D'autres cavités ornées qui comptent généralement peu d'unités graphiques devraient pouvoir, sur la foi de quelques représentations qui rentrent dans la variabilité thématique du Gravettien (techniques propres au Gravettien, empreinte(s) de main(s) négative(s), animaux aux conventions rappelées dans les pages qui précèdent...) être citées : grotte du Bison à Meyrals (Roussot 1984c), Moulin de Laguenay en Corrèze (Couchard 1975 ; Couchard et al. 1984) * et d'autres.

Par une série d'analogies techniques, thématiques, conventionnelles ou "stylistiques ", ces cavités ornées du Quercy cumulent les arguments pour un âge centré sur le Gravettien grâce à une série de recoupements avec les principaux sanctuaires ayant fait, eux, l'objet de dates directes ou indirectes. Cette revue documentaire n'est certainement pas exhaustive et d'autres ensembles pourraient ou devraient s'y joindre comme le Travers de Janoye (Clottes et Lautier 1982) mais la limite avec le Solutréen est délicate à fixer. II faut reconnaître que la question d'un art solutréen en Quercy longtemps évoquée (Leroi-Gourhan 1965 ; Lorblanchet 1972) est aujourd'hui non solutionnée. La tendance étant de regrouper les principaux ensembles au Gravettien, comme guidés par Pech-Merle, elle-même réinterprétée après la découverte de Cussac (Lorblanchet 2004).

\section{2 - Groupe pyrénéen : Le Portel pro parte, les Trois- Frères pro parte}

\section{Grotte du Portel (Plantaurel), phase ancienne}

Même s'il est largement dominé par la période magdalénienne, le dispositif dessiné, peint ou gravé de cette grotte du Plantaurel est loin d'être homogène : dès sa découverte et les premières études (Breuil et al. 1908), les auteurs s'accordent pour y distinguer une phase ancienne. Les conventions morphologiques de plusieurs représentations d'herbivores, notamment des chevaux, montrent des animaux aux corps difformes, aux ventres ballonnés et têtes effilées, émargeant entre les styles II et III de Leroi-Gourhan (1965).

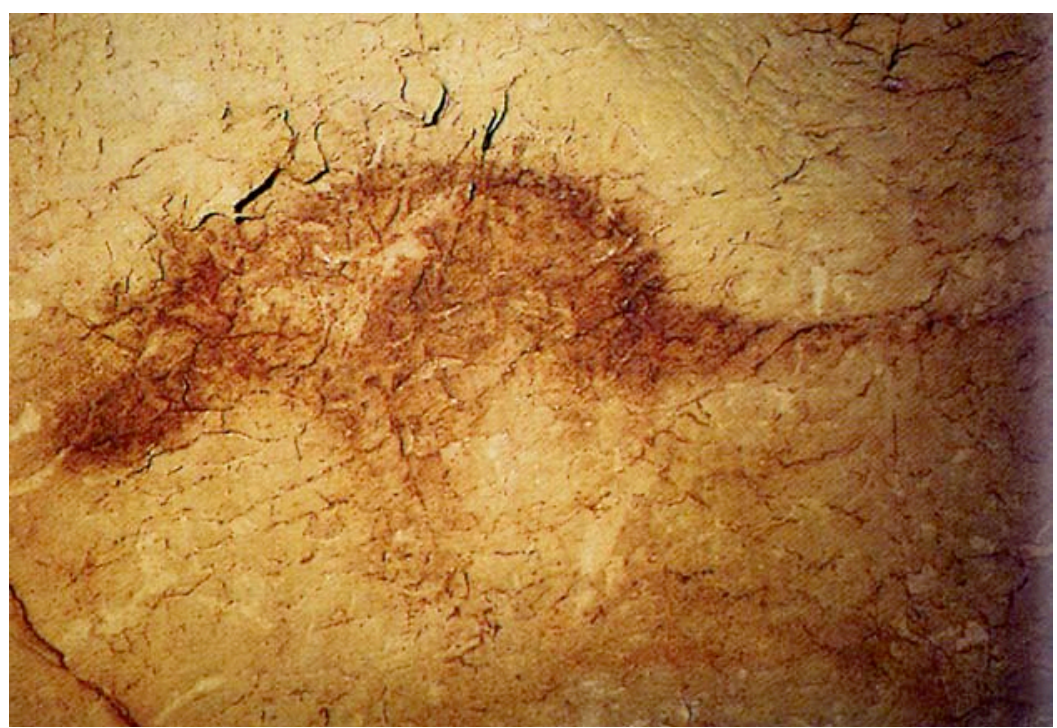

(17) M. Fontugne et al. en cours.
Figure 20 - Grotte du Portel (Loubens, Ariège). Avant-train de cheval réalisé au pochoir brun (@ Lorblanchet 1995). Comparer avec figure 15 Les Merveilles.

Figure 20 - Le Portel cave (Loubens, Ariège). Forehand of a horse painted in brown with stencil (@ Lorblanchet 1995). To be compared with Les Merveilles figure 15 .

\footnotetext{
* Depuis la rédaction de cet article, R. Pigeaud a repris des travaux et obtenu une datation $(26770+380$ BP) pour un échantillon extrait d'un foyer (Pigeaud, 2007).

Bilan scientifique 2006,

DRAC-SRA Limousin, p.17.
} 
Dauvois et Vézian (1984) ont qualifié de nissocéphales (têtes en bec de canard) les grands chevaux bichromes peints au pochoir en brun et rouge - mal conservés - avec, de plus, des crinières en cimier, réalisées par juxtaposition de grosses ponctuations rouges rappelant des conventions observées au Pech-Merle et surtout généralisées à Lascaux. On y joindra le protomé de cheval brun (fig. 20) réalisé au pochoir voisinant avec ce fameux motif rouge dit signe « en accolade » pour Leroi-Gourhan, " aviforme " pour Dauvois et Vézian (op. cit.), la ramure de cerf filiforme rouge si proche de son homologue du Pech-Merle, voisinant avec un autre signe curieux rouge, sorte de main à quatre doigts.

Leroi-Gourhan conclut de manière juste : « (...) à cet égard, je suis tout à fait d'accord pour les situer dans l'espace chronologique qui s'étend de Pair-non-Pair à Lascaux » (op. cit., p. 268). Ce dernier a, par ailleurs, bien montré l'importance de la topographie des différents sanctuaires du Portel en fonction de leur âge supposé, coïncidant avec une assez bonne disjonction spatiale (Leroi-Gourhan 1971 : 267-268) : pied de l'éboulis marquant l'entrée d'origine probable, Grande salle et son prolongement vers le Cloître, début de la Galerie II ou Galerie Jammes. Ces observations sont même à l'origine des travaux de Leroi-Gourhan en matière d'analyse spatiale des grottes ornées qui vont accompagner par la suite l'ensemble de sa réflexion.

Des prélèvements de pigments ont été effectués par $M$. Menu mais non publiés suite à un différend : il serait pourtant intéressant de comparer des dessins noirs tardigla- ciaires (Magdalénien récent) du Portel à dominantes rouge et brune des peintures conventionnelles apposées au pochoir (Gravettien probable).

\section{Grotte des Trois-Frères (Plantaurel)}

Comme pour Le Portel, une partie de l'immense réseau des Cavernes du Volp (Ariège), notamment plusieurs secteurs de la grotte des Trois-Frères, peut être attribuée à une phase ancienne, antérieure au Tardiglaciaire. Les ensembles concernés sont la Galerie des Chouettes, le Panneau du Bison noir et la Galerie dite "aurignacienne" du Tréfonds. Un accès fossile via le Tréfonds est parfois évoqué. Outre les conventions et le style des gravures, un argument souvent avancé sont ces fameux bisons noirs dessinés dans un style magdalénien oblitérant une première génération de motifs relevant d'autres concepts graphiques, dessins dits malhabiles d'herbivores à perspective tordue, cheval à mufle effilé, crinière en cimier, pattes inachevées... (Bégouën et Breuil 1958) (fig. 21). Une bonne partie de cette Galerie aurignacienne montre des figures de ce type que Breuil qualifiait de style « fil de fer ", de même que des dessins sur argile apparentés, eux aussi, à ceux de Gargas (Bégouën et Breuil op. cit). Il faut bien sûr y ajouter les empreintes de mains négatives rouges de la Galerie des Mains que Breuil considérait comme aurignaciennes, analogie avec Gargas oblige.

Les premiers auteurs ont bien montré le dualisme de ces ensembles du Tréfonds où les quelques unités attribuables au Magdalénien sont soit isolées, soit superposées. Leroi-

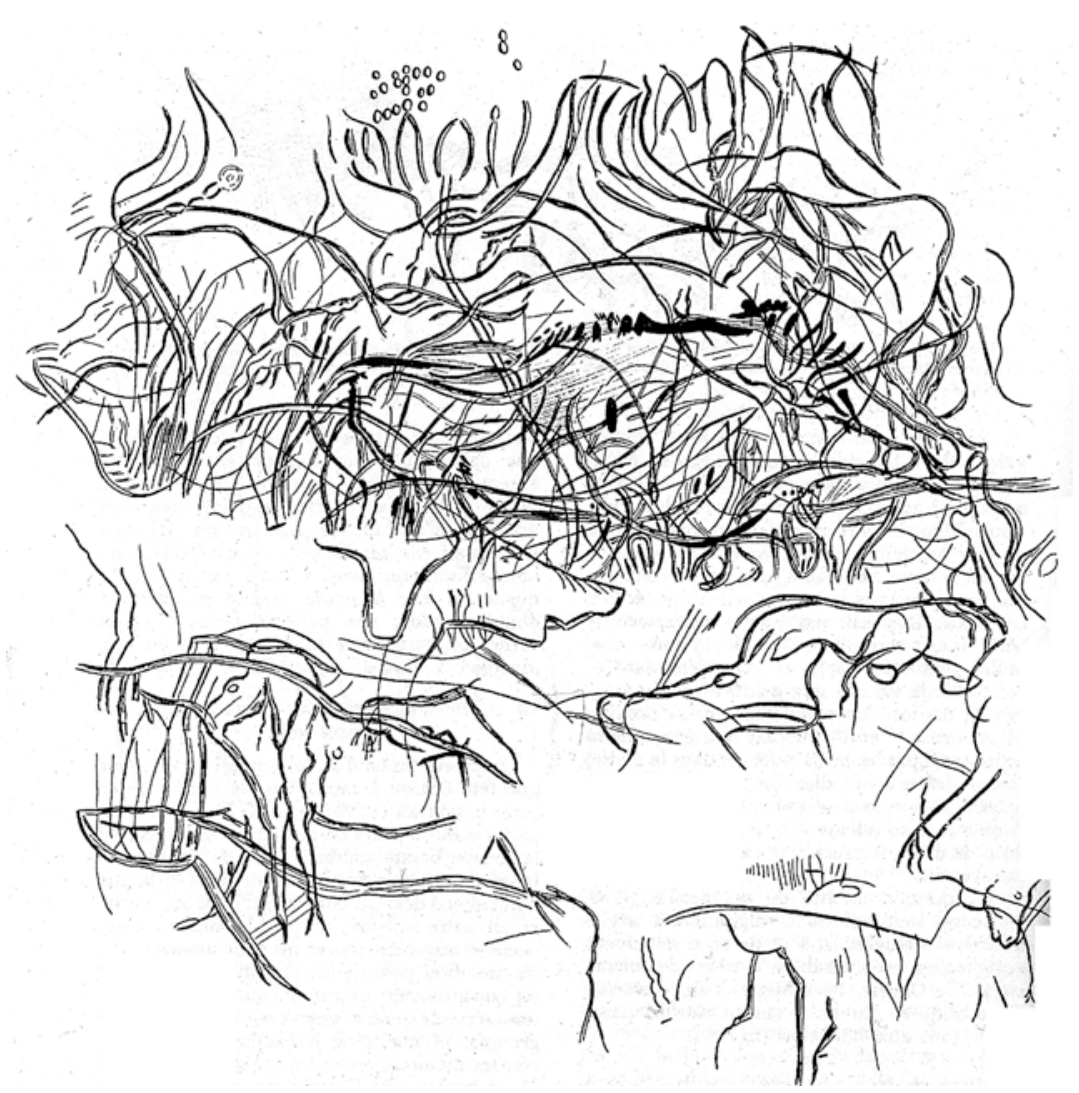

Figure 21 - Grotte des Trois-Frères (Montesquieu-Avantès, Ariège). Tréfonds, Galerie aurignacienne. Dessin noir d'un bison oblitérant un ensemble de gravures d'âge supposé gravettien (relevé H. Breuil in Bégouën et Breuil 1958).

Figure 21 - Trois-Frères cave (Montesquieu-Avantès, Ariège). Subsoil gallery and Aurignacian gallery. Drawing of a bison superimposed on engravings probably dating back to the Gravettian period (tracing $\mathrm{H}$. Breuil in Bégouën et Breuil 1958). 
Gourhan puis Clottes $(1995,2000)$ reprennent cette interprétation dualiste distinguant sans ambiguïté un sanctuaire ancien, d'âge gravettien. L'abbé Breuil insistant sur l'archaïsme des figures penche pour un âge même antérieur à Gargas, d'où le nom de « Galerie aurignacienne ». II serait présomptueux, à partir d'une courte revue documentaire bibliographique et sans en reprendre l'étude avec des outils modernes, de revenir sur les interprétations des maîtres disparus. Disons seulement qu'un ensemble gravettien est quasi-certain (Clottes 1995), ce qui n'est pas le cas de l'Aurignacien.

Rappelons que la zone du Porche d'Enlène (EDG) a livré un mélange de Gravettien moyen à Noailles et de Badegoulien d'abord daté de $21000 \pm 250^{18}$ et $24600 \pm$ 350 (Clottes 1989) et plus récemment par SMA de 27980 \pm 480 BP (Foucher et al. 2001).

\section{3 - Groupe périgourdin : Abri du Poisson, La Grèze, la Mouthe pro parte, Jovelle}

À l'exception de quelques empreintes de mains négatives, l'art probablement gravettien du Périgord est avant tout un art de la gravure.

\section{Abri du Poisson}

L'Abri du Poisson à Gorge d'Enfer (Les Eyzies-de-Tayac, Dordogne) est célèbre pour une magnifique représentation de salmonidé bécard sculpté en léger bas-relief et qui $a$, d'ailleurs, dû être peint (Roussot 1984c). Ce qui l'est moins, hormis de multiples traits gravés peu intelligibles, ce sont des restes de peinture noire et rouge dont se dégage une empreinte de main négative noire (fig. 22) identifiée par Cl. Archambeau et A. Roussot (Roussot 1984c). La séquence incluait un niveau d'Aurignacien ancien surmonté d'un Gravettien à Noailles non daté, lesquels étaient séparés par un cailloutis cryoclastique. La notice de L'Atlas des grottes ornées est aussi précise que claire quant à l'âge gravettien hautement probable de ce petit ensemble (Roussot 1984c). À ceux qui s'étonneraient d'un poisson en bas-relief en contexte gravettien, A. Roussot évoque une technique similaire pour Laussel, à laquelle on ajoutera le probable brochet, dessiné en rouge du Pech-Merle (Lorblanchet 1995).

\section{La Grèze}

Un peu comme le Poisson, cette cavité de la vallée de la Grande Beune (Marquay, Dordogne) a été rendue célèbre par sa seule représentation complète d'un animal, ici un majestueux bison mâle gravé dans un profil absolu, à l'exception de son encornure vue de trois-quarts. B. et G. Delluc (1991) publient d'autres représentations ou segments animaliers moins connus dont un mammouth difforme à ligne ventrale en arche et un mégacéros (fig. 23). $\mathrm{N}$. Aujoulat (1984a) qui a relevé les gravures rappelle que des indices de présence " périgordienne »- au contraire

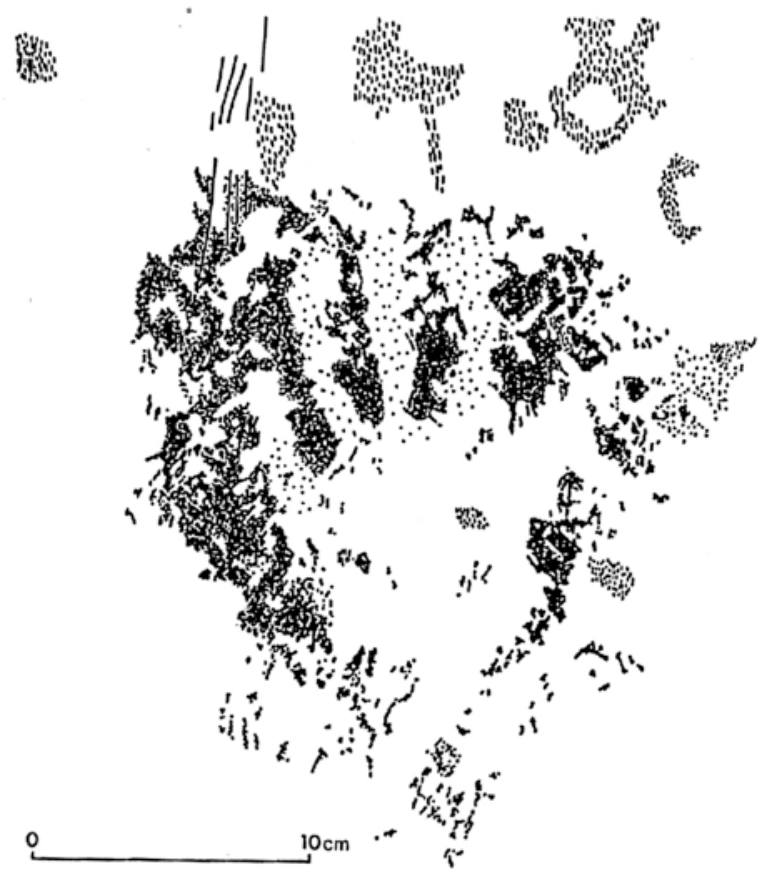

Figure 22 - Abri du Poisson (Les Eyzies-de-Tayac, Dordogne). Relevé de l'empreinte de main négative noire (C B. et G. Delluc 1991).

Figure 22 - Le Poisson rock shelter (Les Eyzies-de-Tayac, Dordogne). Tracing of the black handprint stencil (@ B. et G. Delluc 1991).

du Solutréen - sont attestés par au moins une Font-Robert et un burin de Noailles. On sait par ailleurs que LeroiGourhan citait en exemple le bison de La Grèze pour illustrer son style II, Aujoulat conclut sans être affirmatif que son attribution peut être située entre la seconde partie du «Périgordien supérieur » et le début du Solutréen, ce qui n'est pas le cas de B. et G. Delluc (1991) qui penchent plutôt pour un âge solutréen.

\section{Grotte de La Mouthe pro parte}

On sait que la première salle de la grotte de La Mouthe (Les Eyzies, Dordogne) est à distinguer stylistiquement et techniquement parlant des ensembles graphiques conservés dans les zones profondes de la cavité. La technique privilégie la gravure profonde, le recours aux cupules, une certaine rudesse graphique et les herbivores, aurochs, chevaux ou bisons aux tracés pour le moins sommaires et dépourvus de détails anatomiques ont d'ailleurs été assimilés aux conventions du style II de Leroi-Gourhan (LeroiGourhan 1965 ; Aujoulat et Geneste 1984). Breuil qui a travaillé à La Mouthe a établi des parallèles entre cet ensemble et certains dessins gravés de Pair-non-Pair, d'où l'évocation de l'Aurignacien récent (Breuil 1952). Les attri-

(18) Résultant très probablement d'un mélange (Foucher et al. 2001). 
butions demeurent pour le moins divergentes puisque si Aujoulat et Geneste rappellent que cet ensemble est généralement rapporté au Gravettien ou Proto-Solutréen (1984), B. et G. Delluc (1991) à la suite de leurs relevés préfèrent une attribution plus récente fondée notamment sur l'usage inhabituel sous terre d'une technique réputée archaïque et des indices de mise en perspectives incompatibles avec les corpus d'œuvres d'âge ancien. II faut reconnaître que parmi la pourtant bien longue liste des occupations paléolithiques conservées dans le remplissage du porche d'entrée ne figure apparemment pas le Gravettien. Ainsi La Mouthe n'apparaît pas sur le tableau V de leur conclusion reprenant le décompte des figures animales aquitaines de l'époque archaïque.

\section{L’art pariétal dit « archaïque » d'Aquitaine}

Des grottes ornées comme La Croze à Gontran (Delluc et Delluc 1991), Jovelle (Aujoulat et al. 1984 ; Aujoulat 1984c) ou le modeste ensemble des Bernous relèvent de cet ensemble dit « art pariétal archaïque d'Aquitaine » et, à ce titre, sont rattachés au vaste complexe aurignaco-gravettien. B. et G. Delluc ont longuement présenté des arguments en faveur ou en défaveur d'une attribution soit à l'un (Aurignacien par exemple pour Les Bernous, La Croze à Gontran) soit à l'autre (Gravettien pour Pair-non-Pair, Labattut, le Poisson, Oreille d'Enfer...) et, en l'absence de datations directes, voire de contexte archéologique ou chronologique, ils se sont heurtés comme souvent à la fragilité de tableaux basés sur les seules analyses artistiques. Quelles analogies ou différences doivent être retenues pour rapprocher ou distinguer les équidés de la Croze à Gontran de ceux de Gargas ou du Portel ? Avec ses membres inachevés et raides, son long museau nissocéphale, cette crinière en cimier et un ventre ballonné, le cheval le plus reproduit de la Croze à Gontran trouve en effet une série de similitudes sans équivoques avec des unités graphiques dont l'âge gravettien ne souffre pas la discussion (Labattut, Gargas...). II est cependant réputé aurignacien.

\section{4 - Poitou-Charentes}

En Poitou-Charentes, où les grottes peintes sont rares, J. Airvaux n'attribue qu'un sanctuaire orné au Gravettien, le réseau de Font-Serein à Lussac-les-Châteaux (Vienne). Le dessin au manganèse d'un mammouth grossièrement stylisé, en profil strict, présente des analogies graphiques avec une représentation de pachyderme mais cette fois gravée et sur art mobilier de la grotte du Bouil Bleu en Charente-Maritime (Airvaux 2001). Rappelons que, fort des analogies avec les grottes du Quercy à tectiformes, Lorblanchet (1995) a discuté l'attribution du Placard au Solutréen, à quoi Clottes (2003) lui a sèchement répondu.

\section{5 - Les sanctuaires gravetto-solutréens et tardigra- vettiens du Midi méditerranéen}

À l'exception de Cosquer (seconde phase) et du cas particulier de Chauvet déjà évoqué, aucun des sanctuaires ornés méditerranéens et ardéchois n'est directement daté (sensu 1.1 supra). Dans plusieurs contributions, J. Combier et al. ont bien résumé la chronologie relative de l'art rhodanien avec une "Phase A" qu'ils datent du Solutréen (p. ex. Combier et al. 1960 ; Combier 1989).

\section{Grotte de la Tête-du-Lion (Ardèche)}

La grotte de la Tête du Lion à Bidon, souvent citée comme l'un des meilleurs exemples de panneau peint daté (sensu 1.2 supra) grâce à des charbons au sol (21 $650 \pm 800$ BP, Ly 847) associés à des pigments a conduit à attribuer l'ensemble à une phase ancienne du Solutréen plutôt qu'au « Gravettien final d'ailleurs mal représenté localement » (Combier 1984c, 597). Les analogies avec les œuvres quercinoises du style III à l'époque attribuée au Solutréen renforçant cette interprétation. Nous savons depuis que les dates ${ }^{14} \mathrm{C}$ « classiques " sont souvent plus anciennes, mais, quoi qu'il en soit, même en ${ }^{14} \mathrm{C}$ classique, aucune date du Solutréen inférieur de Laugerie-Haute ne franchit par exemple le cap des 21000 BP (Roque et al. 2001). Or, l'âge obtenu en 1973 indique 21650 avec un $\sigma$ de 800 ! je pense au contraire que la question est plus que jamais d'actualité quant à un éventuel Gravettien final - au moins le Proto-Solutréen - même si les conventions du style III incluant la Frise Noire du Pech-Merle demeurent un bastion solutréen.

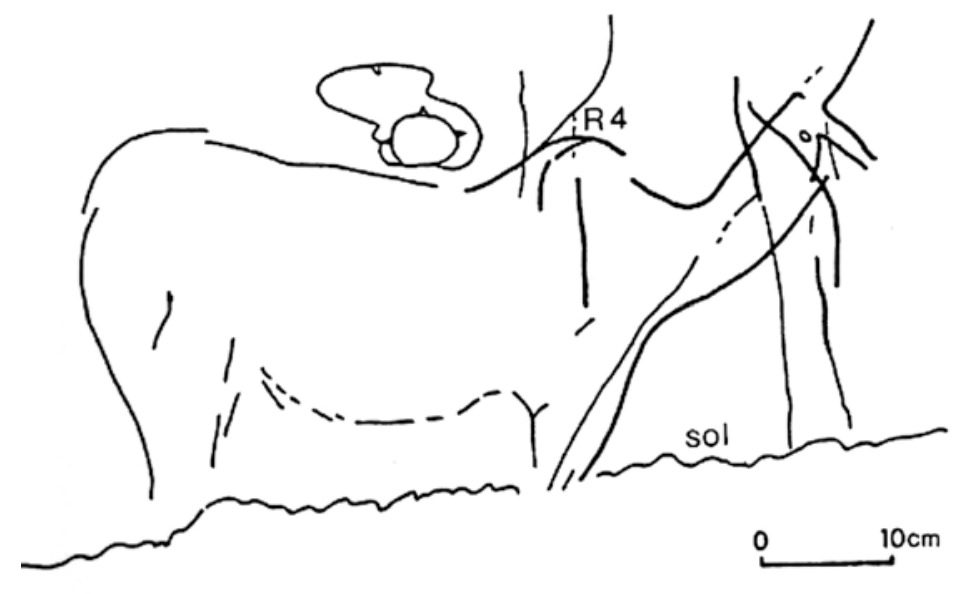

Figure 23 - Grotte de La Grèze (Marquay, Dordogne). Relevé B. et G. Delluc du mégacéros gravé (Delluc 1991).

Figure 23 - La Grèze cave (Marquay, Dordogne). Tracing by B. \& G. Delluc of the engraved giant deer (Delluc 1991). 


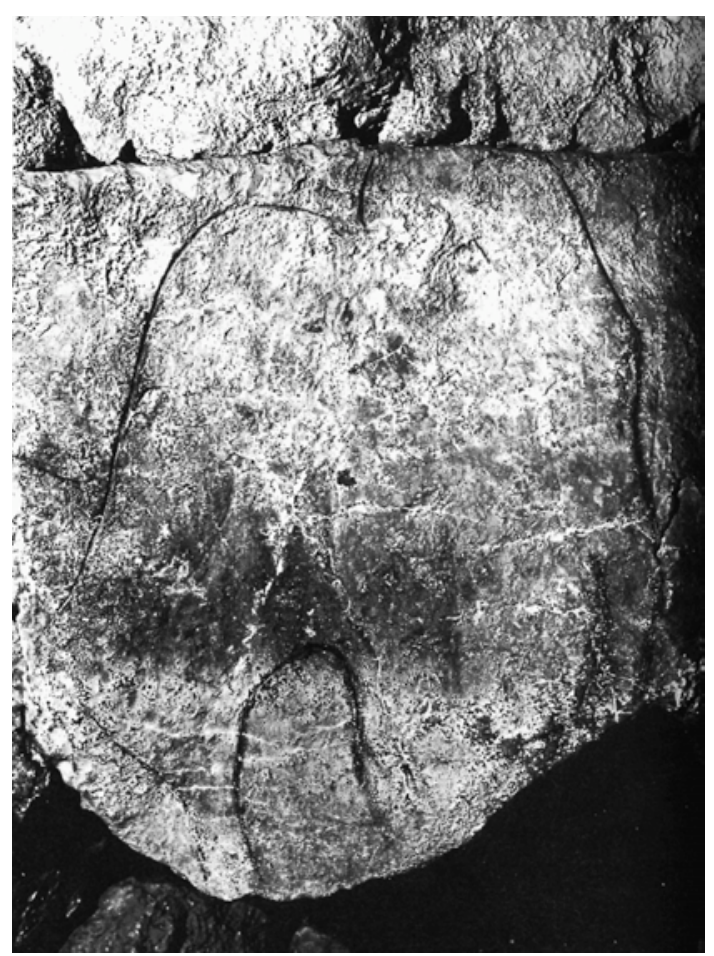

Figure 24 - Grotte Chabot (Aiguèze, Gard). Mammouth gravé (C G. Taupenas, SRA Rhône-Alpes in Combier 1984).

Figure 24 - Chabot cave (Aiguèze, Gard). Engraved mammoth (C) G. Taupenas, SRA Rhône-Alpes in Combier 1984).

\section{Grottes Chabot, d'Oulen (Gard), grotte du Figuier (Ardèche)}

L'ensemble gravé principal de la Grotte Chabot (Gard) montre des conventions stéréotypées d'animaux représentés en profil absolu (style « fil de fer »), avec absence de détails anatomiques, ces fameux mammouths stylisés avec une ligne ventrale en arche (fig. 24), des chevaux à museaux 'becs de canard' que bien des éléments rapprochent du style II (Leroi-Gourhan 1965) reconnu à Gargas, Labattut (Gravettien) mais aussi à la Croze à Gontran (Aurignacien ?) ou Jovelle. Les mammouths y sont stylisés avec un crâne bombé, un dos bossu et une encoche nucale, attribués par Combier au Solutréen (Combier 1984a). II en est de même pour la salle basse et profonde d'Oulen, mais cette fois les mammouths sont peints au doigt (Combier 1984b) (fig. 25). Ou encore l'ensemble du Figuier (Combier et al. 1960 ; Combier 1984d). Pour Oulen, dont l'étude monographique reste à entreprendre ${ }^{19}$, Combier signale des ponctuations, des signes tectiformes ou en accolade, proches de ceux du Pech-Merle, Cougnac, La Pasiega et El Castillo.
Si tous les auteurs s'accordent pour un âge gravetto-solutréen de ces cavités, le distinguo entre les deux complexes, dans la mesure où il peut être opéré en Languedoc oriental où Salpêtrien et Tardigravettien sont par ailleurs attestés, reste à trancher.

\section{Grotte d'Ebbou (Ardèche), Grotte Cosquer, Phase 2 (Tardigravettien ancien)}

Quant à leur voisine Ebbou (Ardèche) l'ensemble gravé est cohérent avec une belle unité de style, des herbivores gravés en profil absolu, avec peu (parfois pas) de détails anatomiques, des têtes petites, voire microcéphales et ces célèbres pattes inachevées en " $X$ ", " $Y$ " (fuseau), autant d'éléments qui les ont rapprochés des styles II-III de LeroiGourhan. Combier et al. ont depuis longtemps établi la comparaison avec l'art mobilier solutréen du Parpalló (Combier et al. 1960) sans que, là non plus, une limite franche puisse être établie avec le Gravettien s.s. (Combier 1989). Cet art pariétal ardéchois qui a effectivement de bonnes chances d'émarger, au moins pour Ebbou, peu ou prou au Solutréen nous mène à nouveau vers le littoral méditerranéen, mais cette fois à la seconde phase de Cosquer.

L'âge est très bien calé grâce à une remarquable série de datations ${ }^{14} \mathrm{C}$ qui en font l'un des ensembles ornés les mieux datés pour le Pléniglaciaire récent (Clottes et Courtin 1994) et des dates qui s'échelonnent entre $19200 \pm 220$ BP et $18010 \pm 190$ BP. Certaines conventions graphiques sont d'ailleurs interchangeables entre Ebbou et ce Tardigravettien de Cosquer (Brochier et Livache 2004), lequel nous rappelle la continuité culturelle plus que probable entre la phase la plus récente du Pléniglaciaire provençal ou ardéchois et le début de l'OIS 2 qui a vu se mettre en place les premiers standards gravettiens.

Avant la découverte de Chauvet, J. Combier ne distinguait pour l'art pariétal d'Ardèche que deux phases, la première étant solutréenne (Chabot, Oulen, Tête du Lion, Bayol...) coïncidant avec le style III et quelques héritages du II de Leroi-Gourhan. Les parallèles qu'il établit systématiquement avec le Quercy, pour justifiés qu'ils soient, nous renvoient à ce difficile distinguo Solutréen/Gravettien. La tendance pour le Quercy étant vers une " gravettianisation 》 de nombreux sites (p. ex. Pech-Merle via Cussac : Lorblanchet 2004) le dossier est toujours ouvert pour la datation de l'art pariétal rhodanien post-Chauvet.

\section{5 - Et Lascaux ?}

Lascaux doit-elle être exclue de cette discussion ? et si non, comment oser aborder - à défaut de régler...! - la question, en quelques lignes, sans être présomptueux ? laquelle n'est pas nouvelle (Glory 1960 : «Les peintures de Lascaux sont-elles périgordiennes ? »). N'est-il pas plus lâche d'éviter le sujet? Nous cédons à la tentation.

(19) R. Pigeaud a récemment déposé une demande de relevé d'art pariétal. 


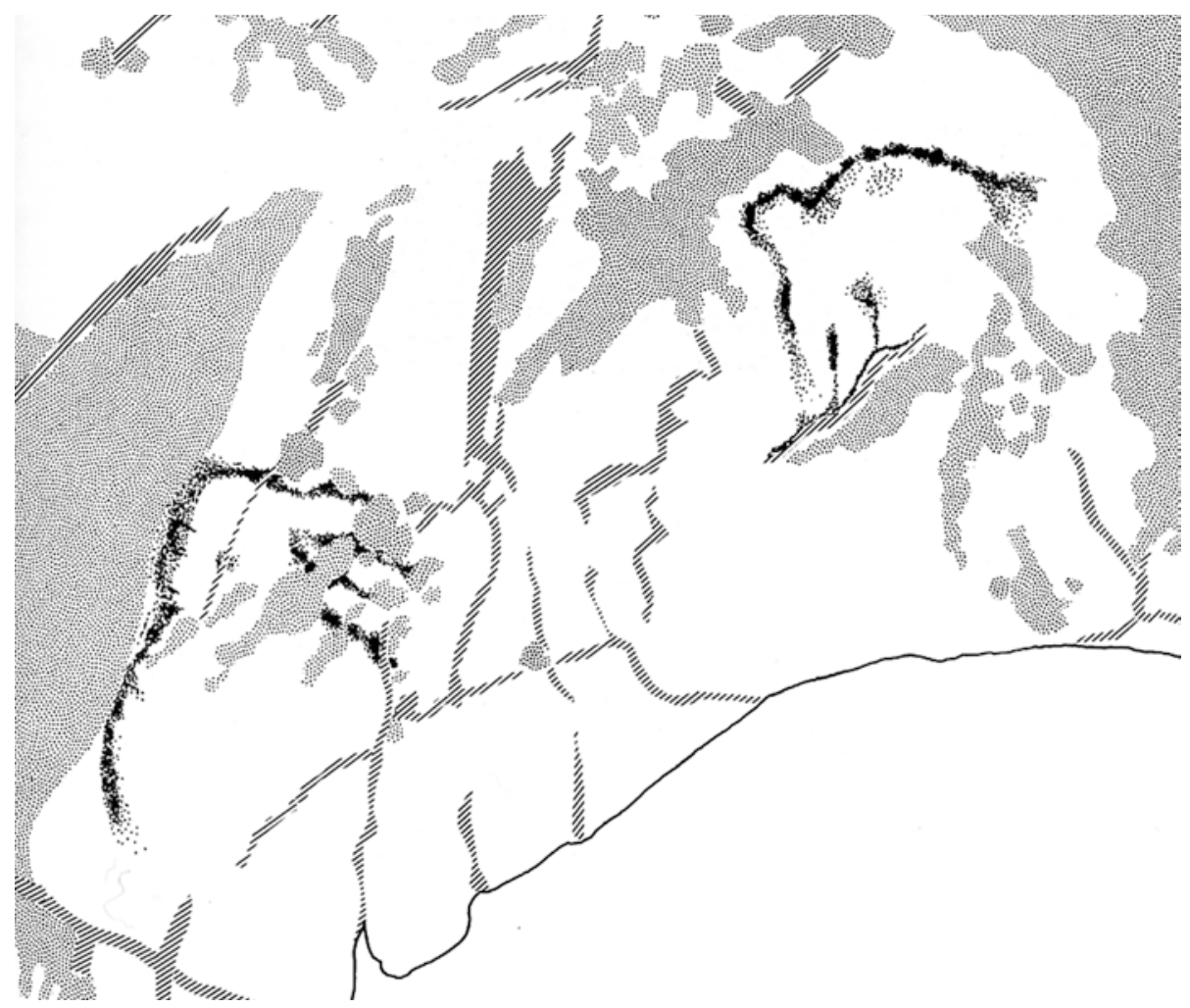

Figure 25 - Grotte d'Oulen (Le Garn, Gard) ; dessins stylisés rouges de deux mammouths réalisés au doigt (relevé J. Combier, dessin P. Ayrolles in Combier 1984).

Figure 25 - Oulen cave (Le Garn, Gard). Red stylized drawings of two mammoths engraved with finger (tracing J. Combier, drawing P. Ayrolles in Combier 1984).

Quels sont les éléments qui peuvent insérer Lascaux dans l'ordre de présentation adopté ici ? Lascaux, manganèse et teintes chaudes obligent n'est pas - et ne sera jamais ? - un sanctuaire assorti de datations radio-numériques directes. Par contre, à défaut d'os fichés, des éléments archéologiques, plus ou moins associés ont fait l'objet de datation. C'est le Puits qui donne l'indice le plus récemment analysé et l'un des plus solide : un fragment de sagaie, découverte au cours des fouilles Breuil, Blanc et Bourgon de 1948 dans le Puits, a donné un âge ${ }^{14} \mathrm{C}$ SMA de $18600 \pm 190$ BP (Aujoulat et al. 1998), soit le Solutréen récent. Si l'on considère ladite sagaie comme effectivement typique de la charnière Solutréen-Badegoulien, ajoutée à la récurrence de conventions graphiques communes et la parenté d'avec l'œuvre de Gabillou, alors il y a effectivement de bonnes présomptions pour situer la décoration de l'un et l'autre à la fin du Solutréen.

Mais Lascaux est complexe. Le dispositif pariétal du Diverticule axial et du Lascaux des peintures sont-il de la même main (sensu de la même génération chrono-culturelle) que l'étrange dessin noir de la Scène du Puits ? Le magnifique travail publié récemment par N. Aujoulat (2004) est suffisamment démonstratif pour nous rappeler certes l'excellence du dispositif pariétal du Lascaux peint, mais dans le même temps toute l'originalité technique de cet art du pochoir, au moins dans les secteurs peints (fig. 26). Et vers quelles références cette analogie technique si forte nous mène-t-elle? Vers le panneau des chevaux ponctués du Pech-Merle et des grands chevaux bruns de la phase ancienne du Portel, notamment. Un consensus place désormais ces derniers au cœur du dispositif gravettien. La technique du soufflé, du pochoir, poussé à son maximum dans le Lascaux peint trouverait-elle sinon une stricte analogie chronologique, du moins quelque origine dans l'art gravettien si bien représenté en Quercy ? S'agit-il de deux phases distinctes? En forçant quelque peu la démonstration pour provoquer la discussion, et sans remonter aux auteurs de la génération Breuil-Peyrony qui attribuaient Lascaux (et Gabillou) au cycle aurignaco-périgordien (Breuil 1952), ne devrait-on distinguer une scène du Puits solutréenne d'un Lascaux peint, sinon gravettien, du moins de tradition gravettienne ? Souvenons-nous aussi de cette plaque peinte de Vignaud découverte dans un niveau à Noailles supra.

Par sa densité iconographique, et aussi le nombre de travaux - donc d'hypothèses - que la cavité a naturellement attirés, Lascaux est trop complexe et trop dense pour que son sort puisse être réglé ici en quelques lignes. Nous souhaitions juste enfoncer un timide coin gravettien dans l'édifice considérant, ces dernières années, Lascaux comme plutôt solutréen. Quoi qu'il en soit, rappelons aussi qu'il y a Gravettien et Gravettien. Pas moins de trois ou quatre millénaires s'écoulent entre un Gravettien moyen à Noailles de Laussel et l'ex-Proto magdalénien - désormais Gravettien final - vers 22000 BP non calibré de Pataud. Pour conclure, disons que, pour nous, l'essentiel du Lascaux polychrome devrait se situer entre un Gravettien récent et le Solutréen récent. 


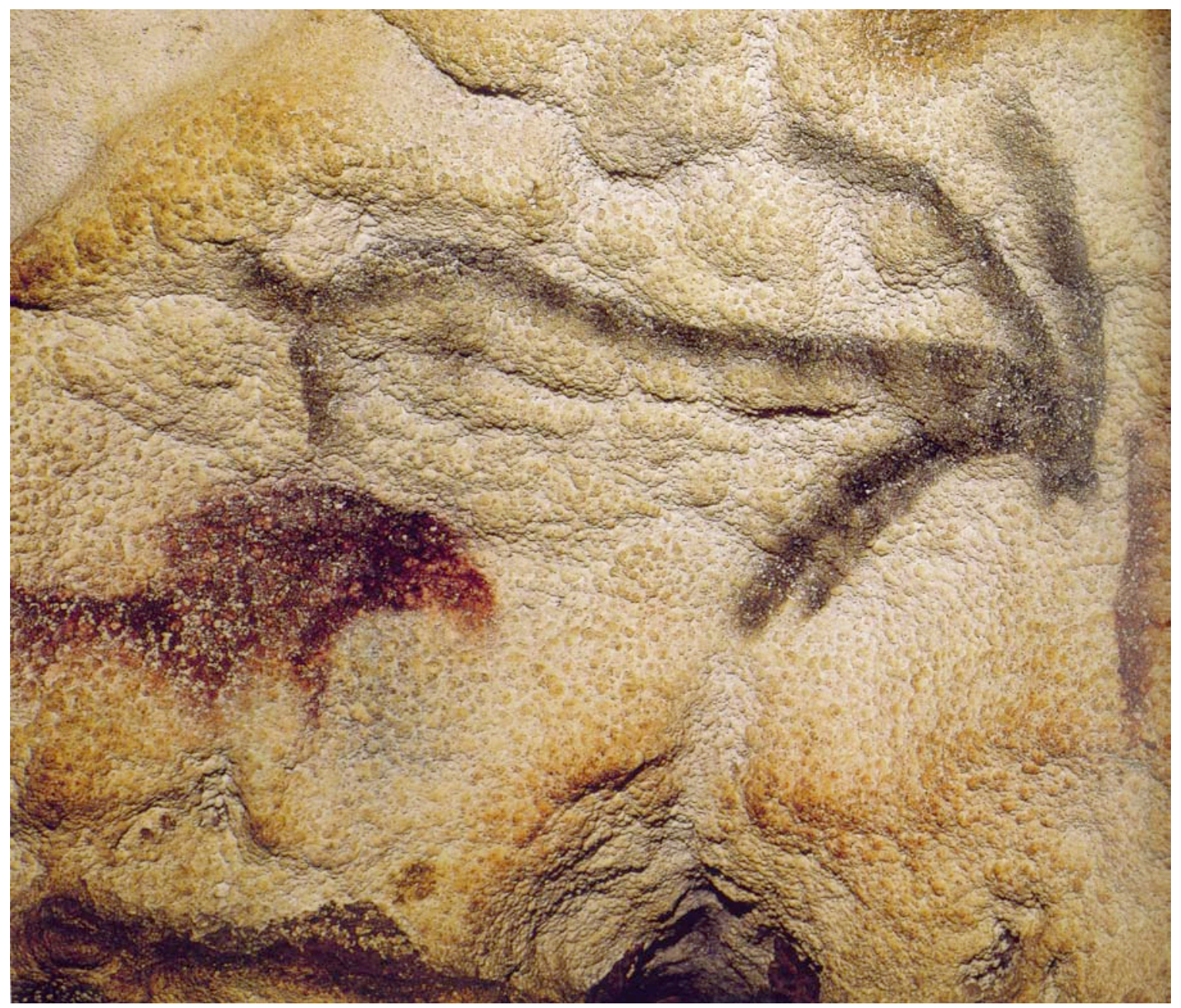

Figure 26 - Grotte de Lascaux (Montignac, Dordogne). Diverticule axial, extrémité gauche du Panneau de la Vache tombant : protomé de cheval réalisé au pochoir brun rouge (comparer avec fig. 20) et bouquetin silhouetté par une série de ponctuations jointives noires (@ Aujoulat 2004).

Figure 26 - Lascaux cave (Montignac, Dordogne). Axial gallery, left end of the Falling Cow panel: forehand of a horse painted in brown with stencil technique, and ibex profile drawn by a series of black joint dots (@ Aujoulat 2004).

\section{4 - SYNTHESE, DISCUSSION}

\section{1 - Bilan diachronique}

\subsection{1 - Radiochronologie « directe»}

D’après le tableau 1, les dates sélectionnées comme supposées gravettiennes s'échelonnent entre $30160 \pm 140$ (Grande grotte d'Arcy) et $18470 \pm 280$ BP pour les niveaux de Gravettien final de Pataud manifestement trop jeunes (fig. 27). On ne peut mettre sur un même plan les dates conventionnelles obtenues jadis sur os, généralement trop récentes et les âges SMA déduits d'analyses d'échantillons non comparables quant à la matière et les proto- coles mis en œuvre. Un lissage et une analyse critique devraient procéder à la pondération de certains âges, peu recevables aujourd'hui ${ }^{20}$.Par ailleurs, les dates sont inégalement représentatives de l'importance des sanctuaires, accordant un éclairage accru aux grottes récemment étudiées (Chauvet, Cosquer) et surtout datables ! L'âge ancien de la grande grotte d'Arcy semble néanmoins acquis, même aux confins de l'Aurignacien récent pour plusieurs dates vers 29-30 ka BP. De même, pour Cosquer, qui relève certainement d'une phase plutôt ancienne du Gravettien (moyenne des dix dates : 26773 BP). PechMerle, Cougnac et Mayenne-Sciences ont donné des âges plus jeunes, vers 24-25 $000 \mathrm{BP}$, voire en continuité avec le Solutréen pour Cougnac.

(20) cf. note 2. 


\subsection{2 - Diachronie relative}

Quant au contexte archéologique, quand il est connu, c'est le Gravettien à Noailles, dont la phase moyenne est située vers 24-26 ka BP qui domine : nous retrouvons le faciès noaillien à Laussel, Labattut, l'Abri du Poisson, Vignaud, Pataud, La Grèze, Gargas et Enlène (cf. TroisFrères). Hormis Les Fieux, les grottes ornées du Quercy sont dépourvues de contexte archéologique. Les dates du Pech-Merle, Cougnac, Cussac et de Mayenne-Sciences ne s'opposeraient pas à une attribution au Noaillien, au contraire.

Par contre, Pair-non-Pair, rejoignant Arcy et Cosquer, devrait précéder ce groupe à Noailles. À l'autre extrémité, il est clair que la longue séquence de Pataud s'achève avec le Gravettien final et ses blocs ornés originaux. La question de la transition Gravetto-solutréenne est parmi les plus passionnantes mais doit être reprise sur la base d'autres approches pour trouver une amorce de solution.

\section{2 - Bilan « artistique »}

\subsection{1 - Techniques}

Une technique aussi spécifique et chargée de contraintes que la peinture aérographique ou projection de colorant par soufflé associé au pochoir, avec ses différentes déclinaisons (Lorblanchet 1980, 1993) nous semble suffisamment originale, et somme toute peu naturelle, pour servir de marqueur. Sans tomber dans le raisonnement circulaire, y aurait-il exclusion, donc monopole de cette technique par les Gravettiens et leurs descendants directs ? Les exemples pléistocènes extra-européens ou ethnographiques répondent sans détour par la négative. À Chauvet, si justement on exclut le petit ensemble de la galerie des Panneaux rouges, le pochoir est absent. D'une manière générale, les Magdaléniens n'ont pas été des adeptes de telles pratiques. Malheureusement, les données manquent le plus souvent pour être catégorique, mais il y a ici une voie des plus intéressantes. Un peu comme le modelage d'argile s'associe au Magdalénien pyrénéen.

Le reste des techniques paraît trop courant et par trop ubiquiste pour être sollicité : gravures, dessins au trait peint ou gravé. Si l'on exclut Lascaux du Gravettien, la polychromie demeure rare ou plus tardive. Si le bas-relief commence un parcours notoire avec le Gravettien (Laussel, Abri du Poisson) il s'épanouira avec le Solutréen (Fourneau-du-Diable, Roc-de-Sers) et atteindra l'apogée qu'on lui connaît au Tardiglaciaire magdalénien.

\subsection{2 - Bestiaire}

Des herbivores du bestiaire pléistocène, certaines espèces sont de bons indicateurs chronologiques : la représenta- tion du mégacéros par exemple franchit-elle le cap du Solutréen ? Quelque temps après la découverte de Chauvet, Clottes $(1995,2000)$ note les différences qui marquent le passage du bestiaire aurignacien au Gravettien. On y ajoutera la présence du Mammouth, ou plutôt d'un certain Mammouth aux conventions maintes fois décrites (p. ex. Breuil 1952 ; Leroi-Gourhan 1965 ; Combier et al. 1958 ; Delluc et Delluc 1991...). Les mammouths de la période ancienne semblent nous éloigner du Pech-Merle (Frise Noire) et surtout de Lascaux où ils brillent par leur absence. $Y$ aurait-il un Gravettien avec mammouth type Jovelle - Chabot et une seconde phase apparemment plus récente où le mammouth glabre, stylisé et filiforme laisserait la place à des pachydermes à toison (Cussac, Frise noire du Pech-Merle) mieux détaillés ? Ces différences ont-elles une autre signification que leur âge?

Un argument encore trop peu mobilisé pour la datation de Lascaux est la présence d'un bestiaire plutôt tempéré ${ }^{21}$, quelle qu'en soit par ailleurs sa signification, par exemple saisonnière (Aujoulat 2004). Passons sur le Cheval, herbivore omniprésent tout au long du Paléolithique récent, l'Aurochs et le Cerf qui le suivent dans les décomptes (Aujoulat, 2004 : 64-65) signent un environnement peu rigoureux, pour ne pas dire forestier, loin de l'image du DMG coïncidant avec le Solutréen, pas plus qu'avec le Gravettien final. Certes, on ne connaît pas dans le détail l'évolution des faunes en fonction de courts répits des événements Dansgaard-Oeschger 4, 3 ou 2. Un décalage peut toujours exister entre environnement et bestiaire cultuel, mais on ne pourra échapper à certaines lois. Le travail reste à faire.

\subsection{3 - Représentations animales}

Cela a été dit et écrit, l'art animalier gravettien est un art de la silhouette, emprunt de figuratif synthétique, voire stylistique (style « fil de fer » de Breuil), avec traitement géométrique de certaines parties du corps (Guy 2004), la célèbre perspective semi-tordue de Breuil, la rareté voire l'absence de détails anatomiques, un désintérêt relatif pour les extrémités ou le pelage (toison des mammouths souvent glabres - avec de notables exceptions pour Cussac). Les corps sont le plus souvent difformes, aux ventres ballonnés, des plis unguéaux exagérés et une accentuation de la ligne cervico-dorsale. En ce qui concerne la tête et l'encolure, on note une tendance à la microcéphalie, des crinières en cimier, parfois échevelées, des cous col de cygne et le non moins fameux museau bec de canard, aux mufles tombants de l'abbé Glory, les figurés nissocéphales de Dauvois ou encore les museaux tréflés des félins décrits par Lorblanchet. L'implantation de l'oreille est également peu conforme à la réalité. Enfin, pour les membres et la queue, c'est le domaine de l'inachevé, avec bien souvent unicité des pattes par paire (É. Guy), des représentations de pattes en « $X$ » ou en « $Y$ » et une queue linéaire.

(21) Cf. à ce sujet la discussion in Clottes 2003 qui réfute l'hypothèse de Bahn qui se sert de l'Aurochs pour écrire que les taureaux de Lascaux pourraient être holocènes. Comme Biache-Saint-Vaast et La Borde sans doute! 


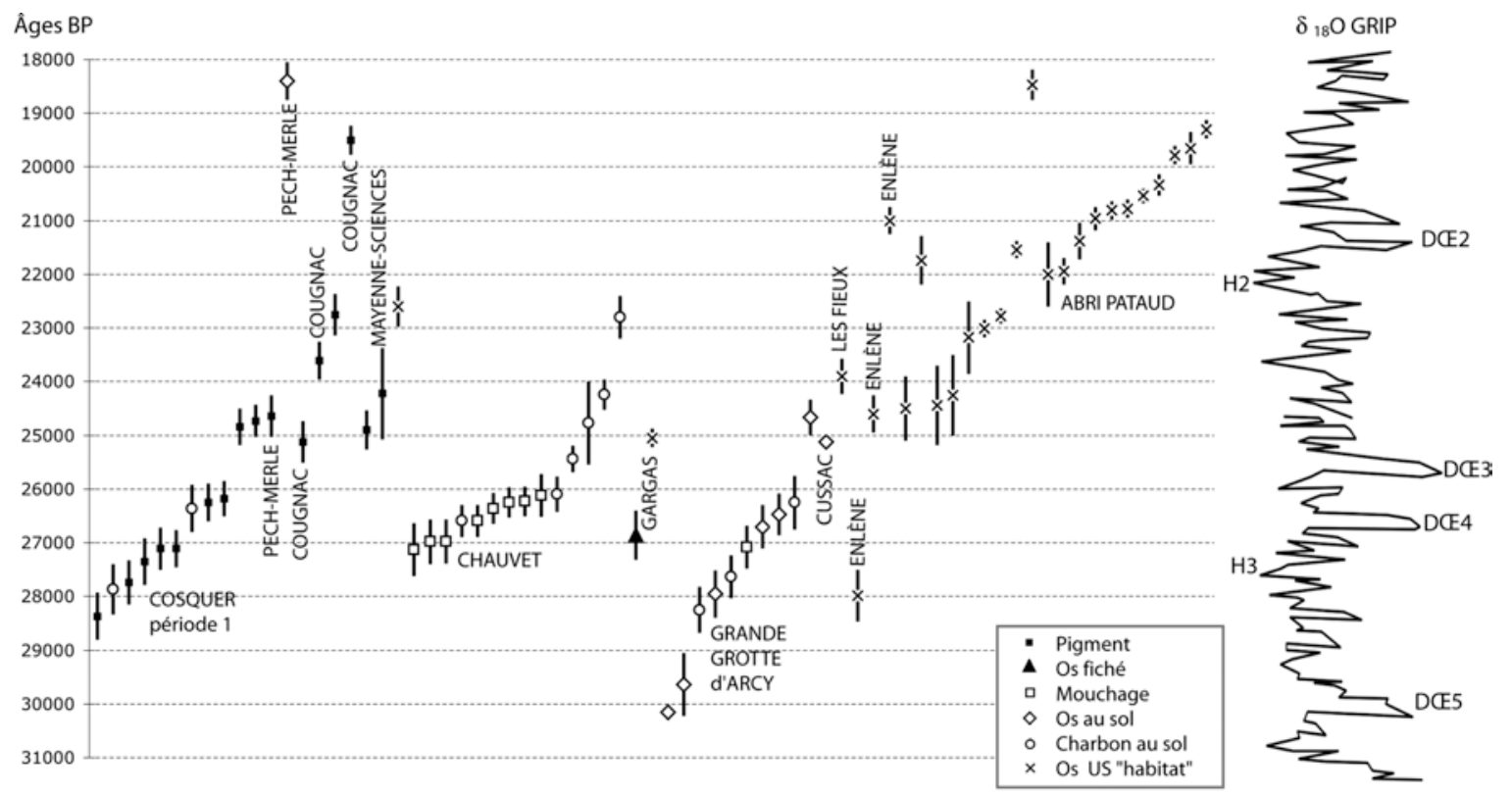

Figure 27 - Dates ${ }^{14} \mathrm{C}$ exprimées en âges BP des sanctuaires ornés gravettiens (France uniquement) ou supposés tels. Sur la droite, courbe $\delta^{18} \mathrm{O}$ GRIP (Groenland) entre 18 et 31 ka $B P(H=$ événements de Heinrich ; $D$ - $C E$ = interstadiaires DansgaardOeschger.

Figure 27 - BP C14 dates for Gravettian painted caves or those ascribed to this period (only France). On the right, GRIP $\delta^{18} \mathrm{O}$ sequence (Greenland) between 18 and 13 kyr BP ( $H=$ Heinrich events; $D-C E$ : Dansgaard-Oeschger stadials).

\subsection{4 - Thèmes}

La représentation de corps humains est un élément fort de l'art gravettien, mais elle demeure avant tout mobilière par la géographie emblématique des figurines dites « vénus ». Nous avons cependant avec Laussel un magnifique relais quant au support entre mobilier et pariétal. Gravées en voûte sur le Plafond des Hiéroglyphes du Pech-Merle, peint sur le panneau des Femmes-Bisons ou gravées grand format à Cussac, il a été clairement montré la parenté évidente du thème, du figuré et des conventions pour réunir les deux sanctuaires (Aujoulat et al. 2004).

Le thème de l' " homme blessé » n'est clairement attesté qu'à Cougnac et Pech-Merle, ceux de Cosquer étant équivoques. Ils ne sont pas directement datés du Gravettien, mais apparaissent dans des sanctuaires ornés où les principaux panneaux ont donné des âges antérieurs au Solutréen. Si les deux cavités quercinoises basculent en bloc dans un Gravettien récent, ce qui n'est encore qu'une hypothèse, alors que dire des signes aviformes également associés, longtemps passés pour solutréens ? Les signes du Placard ont eux été rapportés au Solutréen sur la foi d'éléments gravés découverts en stratigraphie dans un niveau de Solutréen supérieur (Clottes et al. 1990). Mais il s'agit ici d'un terminus a quo, non ad quem, l'âge solutréen récent devant être considéré comme un minimum (Clottes et al. 1991) même si la séquence solutréenne est dilatée. Clottes (2003) rappelle avec force que le Gravettien est absent du Placard.
Les empreintes de mains ont retenu l'attention de la plupart des spécialistes qui en ont de tout temps fait un thème incontournable de " l'art " gravettien ou assimilé. Elles sont en effet presque toujours en contexte gravettien et, par exemple, il n'y a pas à notre connaissance d'ensembles ornés riches en empreintes de mains négatives en contexte typiquement et uniquement magdalénien. En poursuivant sur la même voie, le thème "empreinte de mains aux phalanges manquantes " renforce l'attribution au Gravettien (Gargas, Tibiran, Cosquer, Grande Grotte d'Arcy) même si la date de Fuente del Salín (22 $340 \pm$ $510 / 480$ BP) est un peu jeune. II en est de même pour les pochoirs de pouces ou index repliés du Sanctuaire des mains de Gargas et des chevaux ponctués du Pech-Merle (Clottes et Courtin 1994 : 167 ; Clottes 1995).

Les séries de grosses ponctuations toujours réalisées au soufflé (Pech-Merle, Le Combel, Frayssinet, Les Merveilles...) paraissent émerger dans la même gamme, tant technique que thématique. En cela, le Diverticule axial de Lascaux se rapproche ponctuellement du Gravettien.

\subsection{5 - Homogénéité versus hétérogénéité}

À parcourir cet art gravettien, un constat s'impose : une homogénéité évidente caractérise un certain nombre de sites décrits et interprétés comme tels (Cussac, Pair-nonPair, la Grande Grotte d'Arcy, Mayenne-Sciences). Cette impression première est rapidement nuancée par l'hétérogénéité d'ensembles probablement plus complexes, du 
moins peu évidents, plus difficiles à dater, aux fréquentations depuis longtemps discutées (p. ex. Pech-Merle, Lascaux). Cussac devrait nous faire avancer. Un troisième ensemble serait représenté par des sanctuaires à l'hétérogénéité évidente et reconnue mais où la phase gravettienne est minoritaire : Cosquer dominée par les unités graphiques contemporaines du DMG, Le Portel et les TroisFrères dominés par le Magdalénien, etc. Enfin, un dernier ensemble de cavités apparaît de prime abord comme des plus homogène mais certains résultats discordants, notamment des datations, les situent parfois au cœur de discussions assez vives comme Cougnac (Lorblanchet 1994 ; Clottes 1995, 2003 ; Sauvet 2004), voire Chauvet (Aurignacien) et ses fameux mouchages de torches postérieurs à l'Aurignacien et, pour certains, quelques éléments supposés postérieurs (Guy 2004).

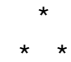

II serait tentant - mais dangereux - de forcer à tout prix l'attribution d'ensembles ornés au Gravettien. Force est de constater que la distinction d'un corpus gravettien, soidisant homogène, d'avec l'Aurignacien d'un côté, d'une continuité plus que probable avec le Solutréen de l'autre est souvent difficile, parfois délicate et plus généralement impossible. En faisant exploser peu ou prou l'évolution linéaire des styles, conventions ou techniques, la découverte et la datation peu douteuse de Chauvet à l'Aurignacien nous a appris ou réappris la prudence dans ce domaine. D'où cet essai un peu sec basé avant tout sur les éléments radio-chronologiques et archéo-stratigraphiques.

\section{BIBLIOGRAPHIE}

AIRVAUX J. 2001 - L'Art préhistorique du PoitouCharentes. Sculptures et gravures des temps glaciaires. Paris : La maison des roches éd., 223 p.

AUJOULAT N. 1984a - Grotte de la Grèze. L'Art des Cavernes. Atlas des grottes ornées paléolithiques françaises. Paris : Ministère de la Culture, Imprimerie Nationale, p. 164-166.

AUJOULAT N. 1984b - Grotte du Roc de Vézac. L'Art des Cavernes. Atlas des grottes ornées paléolithiques françaises. Paris : Ministère de la Culture, Imprimerie Nationale, p. 242-244.

AUJOULAT N. 1984c - Grotte de Jovelle. L'Art des Cavernes. Atlas des grottes ornées paléolithiques françaises. Paris : Ministère de la Culture, Imprimerie Nationale, p. 236-238.

AUJOULAT N. 2004 - Lascaux. Le Geste, l'Espace et le Temps. Paris : Éd. du Seuil, coll. « Arts rupestres », 273 p.

AUJOULAT N., BÉGOUËN R., CLOTTES J., DELPECH Fr., GAUSSEN J., GENESTE J.-M., LAVILLE H., LORBLAN-
CHET M., RIGAUD J.-Ph., ROUSSOT A. 1984 Découverte de gravures dans la grotte de Jovelle (Dordogne). Bull. de la Soc. Préhist. Franç., 81, 70-73.

AUJOULAT N., CLEYET-MERLE J.-J., GAUSSEN J., TISNERAT N. et VALLADAS H. 1998 - Approche chronologique de quelques sites ornés paléolithiques du Périgord par datation carbone 14 en spectrométrie de masse par accélérateur de leur mobilier archéologique. Paléo, 10, déc. 1998, p. 319-323.

AUJOULAT N., FRITZ C. et TOSELLO G. 2001 - La galerie des Panneaux rouges. In Clottes (dir.), La grotte Chauvet. L’Art des origines. Le Seuil, éd. Paris, p. 77-86.

AUJOULAT N. et GENESTE J.-M. 1984 - Grotte de la Mouthe. L'Art des Cavernes. Atlas des grottes ornées paléolithiques françaises. Paris : Ministère de la Culture, Imprimerie Nationale, p. 144-147.

AUJOULAT N., GENESTE J.-M., ARCHAMBEAU Ch., DELLUC M., DUDAY H., GAMBIER D., 2001 - La Grotte ornée de Cussac (Dordogne) ; Observations liminaires. Paleo, $\mathrm{n}^{\circ} 13$, déc. 2001, p. 9-18.

AUJOULAT N., GENESTE J.-M., ARCHAMBEAU Ch., DELLUC M., DUDAY H. et HENRY-GAMBIER D. 2004 - La grotte ornée de Cussac - Le Buisson-de-Cadouin (Dordogne) : premières observations. Bull. de la Soc. Préhist. Franç., 99, 1, p. 129-137.

AUJOULAT N., GENESTE J.-M., ARCHAMBEAU Ch., DELLUC M., DUDAY H. et GAMBIER D. 2004 - La grotte ornée de Cussac. Le Buisson-de-Cadouin (Dordogne). In M. Lejeune et A.-C. Welté (dir.), L'Art pariétal paléolithique dans son contexte naturel. Actes du colloque 8.2 , Congrès de I'UISPP, Liège 2-8 sept. 2001, p. 45-53 (ERAUL, 107).

BAFFIER D. et GIRARD M. 1992 - La Grande grotte d'Arcy-sur-Cure (Yonne), nouveau sanctuaire paléolithique. Revue Archéo. de l'Est et Centre-Est, CNRS Éd., 43 , p. $195-205$.

BAFFIER D. et GIRARD M. 1995a - La Grande grotte d'Arcy-sur-Cure (Yonne) second sanctuaire paléolithique bourguignon. L'Anthropologie, 99, 2/3, p. 212-220.

BAFFIER D. et GIRARD M. 1995b - Les sanctuaires d'Arcy-sur-Cure. Archéologie Nouvelle, 17, Déc.-Janv. 1995, p. 47-49.

BAFFIER D. et GIRARD M. 1998 - Les cavernes d'Arcysur-Cure. Paris : La maison des roches édit., 120 p. (Coll. «Terres préhistoriques »).

BARD É., ARNOLD M. HAMELIN B., TISNERAT-LABORDE N. and CABIOCH G. 1998 - Radiocarbon Calibration by means of Mass Spectrometric ${ }^{230} \mathrm{Th} /{ }^{234} \mathrm{U}$ and ${ }^{14} \mathrm{C}$ Ages of corals: an updates database including samples from Barbados, Mururora and Tahiti. Radiocarbon, 40, 3, p. 1085-1092. 
BARRIÈRE Cl. 1976 - L'art pariétal de la grotte de Gargas. Oxford : BAR, International series, S14 et Toulouse : Mémoire de l'Institut d'Art Préhist., III, 2 vol., 409 p.

BÉGOUËN H. et BREUIL Abbé H. 1958 - Les Cavernes du Volp. Trois-Frères - Tuc d'Audoubert à MontesquieuAvantès (Ariège). Paris : Art et Métiers Graphiques, 124 p. (Travaux de l'Institut de Paléontologie humaine).

BREUIL H. 1952 - Quatre cents siècles d'art pariétal. Montignac : Centre d'Études et de Documentation préhistoriques, $413 \mathrm{p}$.

BREUIL H. et CHEYNIER A. 1958 - Les fouilles de Breuil et Cartaillac dans la grotte de Gargas en 1911 et 1913. Toulouse : Bull. de la Société Méridionale de Spéléologie et de Préhistoire, V (1954-55), Bull. de la Soc. d'Hist. Nat. de Toulouse, 93, 1958, Privat édit., p. 341-382.

BREUIL H., JAMES C., JEANNEL R. 1908 - Les dernières peintures découvertes à la grotte du Portel (Ariège). C. $R$. Acad. Sciences de Paris, 46, p. 1166-1168.

BRICKER H. M., dir. 1995 - Le Paléolithique supérieur de l'abri Pataud (Dordogne) : les fouilles de H. L. Movius Jr., Paris : Maison des Sciences de l'Homme, 328 p. (Doc. d'Archéo. Franç., 50).

BRICKER H. M., BROOKS A. S., CLAY R. B., DAVIS N. 1995 - Les fouilles de H. L. Movuis Jr. L'abri Pataud : généralités. In : Bricker H. M., dir., Le Paléolithique supérieur de l'abri Pataud (Dordogne) : les fouilles de H. L. Movius Jr., Paris : Maison des Sciences de l'Homme, p. 11-29 (Doc. d'Archéo. Franç., 50).

BROCHIER J.-É. et LIVACHE M. 2003 - Les niveaux à crans de l'abri du Rouet (Carry-le-Rouet, Bouches-duRhône) et les industries pléni-tardiglaciaires du bassin basrhodanien. In E. Ladier (dir.). Les pointes à cran dans les industries lithiques du Paléolithique supérieur récent de l'oscillation de Lascaux à l'oscillation de Bölling. Actes de la table-ronde de Montauban (Tarn-et-Garonne) 26-27 avril 2002, p. 47-66 (Suppl. à Préhist. du Sud-Ouest, 6).

CHAMPAGNE F., JAUBERT J. 1986 - Un exemple de remplissage archéologique en milieu karstique : la grotte des Fieux, à Miers (Lot). Toulouse : Bull. de la Soc. Mérid. de Spéléologie et Préhistoire, XXVI, p. 21-33.

CHAMPAGNE F., CHAMPAGNE Ch., JAUZON P., NOVEL Ph. 1990. Le site préhistorique des Fieux à Miers (Lot). État actuel des recherches. Gallia Préhistoire, 32, p. 1-28.

CHEYNIER Dr. A. et BREUIL Abbé H. (coll. M. E. Boyle, R. L. Doize) 1963 - La caverne de Pair-non-Pair. Gironde. Fouilles de François Daleau. Bordeaux : Publ. de la Soc. Archéo. de Bordeaux, 215 p., pl. h.-t. (Documents d'Aquitaine, II).

CLOTTES J. 1989 - Le Magdalénien des Pyrénées. In : Le Magdalénien en Europe. La structuration du Magdalénien.
Actes du colloque de Mayence 1987, Xle congr. de I'UISPP, p. 281-360 (ERAUL, 38).

CLOTTES J. 1995 - Changements thématiques dans l'art du Paléolithique supérieur. Préhistoire ariégeoise, Bull. de la Soc. Préhistorique Ariège-Pyrénées, L, p. 13-34.

CLOTTES J. 2000 - Art between 30,000 and 20,000 bp. In W. Roebroeks, M. Mussi, J. Svoboda \& K. Fennema Eds., Hunters of the Golden Age. The Mid Upper Palaeolithic of Eurasia 30,000 - 20,000 BP, Leiden: University of Leiden, p. 87-103.

CLOTTES J. 2003 - Un problème de parenté : Gabillou et Lascaux. In : "Préhistoire et sociétés », Mélanges Jean Gaussen. Bull. de la Soc. Préhist. Ariège-Pyrénées, LVIII, p. 47-61.

CLOTTES J., CHAUVET J.-M., BRUNEL-DESCHAMPS É., HILLAIRE Ch., DAUGAS J.-P., ARNOLD M., CACHIER H., ÉVIN J., FORTIN P., OBERLIN Ch., TISNERAT N., VALLADAS H. 1995 - Les peintures paléolithiques de la grotte Chauvet à Vallon-Pont-d'Arc (Ardèche, France) : datations directes et indirectes par la méthode du radiocarbone. C.R. Acad. Sc. de la Vie, 320, série lla, p. 1133-1140.

CLOTTES J. et COURTIN J. 1994 - La grotte Cosquer. Peintures et gravures de la caverne engloutie. Paris : Le Seuil éd., 197 p. (Coll. « Arts rupestres »).

CLOTTES J., COURTIN J.,VALLADAS H., CACHIER H., MERCIER N., ARNOLD M., 1992. La Grotte Cosquer datée. Bull. Soc Préhist. Franç., 89, 8, p. 230-234.

CLOTTES J., COURTIN, J. et VANRELL L. 2005 - Cosquer redécouvert. Paris : Le Seuil éd., 255 p. (Coll. "Arts rupestres $"$ ).

CLOTTES J., DUPORT L., FERUGLIO V. 1990 - Les signes du Placard. Préhistoire ariégeoise, Bull. de la Soc. Préhistorique Ariège-Pyrénées, XLV, p. 15-49.

CLOTTES J., DUPORT L., FERUGLIO V. 1991 - Derniers éléments sur les signes du Placard. Préhistoire ariégeoise, Bull. de la Soc. Préhistorique Ariège-Pyrénées, XLVI, p. 119-132.

CLOTTES J. et LAUTIER J. 1982 - La grotte ornée paléolithique du Travers de Janoye, à Penne (Tarn). In : "La préhistoire du Quercy dans le contexte de Midi-Pyrénées ", Congr. Préhist. de France, XXle session MontaubanCahors, Septembre 1979, p. 105-115 (Vol. 1).

COLLINA-GIRARD J. 1999 - Réseaux de vallées et prospection sismique : relations et implications structurales dur le plateau continental (Marseille, Bouches-du-Rhône). C.R. Acad. Sc. Paris, 329, p. 357-362.

COMBIER J. 1984a - Grotte Chabot. L'Art des Cavernes. Atlas des grottes ornées paléolithiques françaises. Paris : Ministère de la Culture, Imprimerie Nationale, p. 317-322. 
COMBIER J. 1984b - Grotte d'Oulen. L'Art des Cavernes. Atlas des grottes ornées paléolithiques françaises. Paris : Ministère de la Culture, Imprimerie Nationale, p. 327-332.

COMBIER J. 1984c - Grotte de la Tête-du-Lion. L'Art des Cavernes. Atlas des grottes ornées paléolithiques françaises. Paris : Ministère de la Culture, Imprimerie Nationale, p. 595-599.

COMBIER J. 1984d - Grotte du Figuier. L'Art des Cavernes. Atlas des grottes ornées paléolithiques françaises. Paris : Ministère de la Culture, Imprimerie Nationale, p. 600-604.

COMBIER J. 1989 - À propos de la chronologie de l'art pariétal rhodanien. In : L'art pariétal paléolithique. Paris : Actes des colloques de la Direction du Patrimoine, Périgueux-Le Thot, Nov. 1984, p. 115-116.

COMBIER J., DROUOT É., HUCHARD P. 1960 - Les grottes solutréennes à gravures pariétales du canyon inférieur de l'Ardèche. Mémoires de la Soc. Préhist. Franç., V (1958), p. 61-117.

COUCHARD J.-L. 1976 - La Grotte du Moulin de Laguenay et ses peintures préhistoriques. Bull. de Soc. Scient., Hist. et Archéol. de la Corrèze, 98, 43-47.

COUCHARD J.-L., Groupe spéléologique de la Corrèze, Mazière G. et Raynal J.-P. 1984 - Grotte du Moulin de Laguenay. L'Art des Cavernes. Atlas des grottes ornées paléolithiques françaises. Paris : Ministère de la Culture, Imprimerie Nationale, p. 355-357.

COUSSY P. 2005 - Roucadour, l'art initial gravé. Éd. Résurgences, Cajarc, Impr. France-Quercy, Cahors, 1 vol. n.p.

DAUVOIS M. et VÉZIAN J. 1984 - Grotte du Portel. L'Art des Cavernes. Atlas des grottes ornées paléolithiques françaises. Paris : Ministère de la Culture, Imprimerie Nationale, p. 381-388.

DELLUC B. et DELLUC G. 1978 - Les manifestations graphiques aurignaciennes sur support rocheux des environs des Eyzies. Gallia Préhistoire, 21, 1-2, p. 213-438.

DELLUC B. et DELLUC G. 1979 - La grotte ornée des Bernous à Bourdeilles (Dordogne). Bull. de la Soc. Préhist. Franç., 76, 2, p. 39-44.

DELLUC B. et DELLUC G. 1987 - Petit bloc portant une tête de cerf de l'abri Vignaud aux Eyzies. Bull. de la Soc. Hist. Archéo. du Périgord, CXIV, p. 258-259.

DELLUC B. et DELLUC G. 1991 - L'Art pariétal archaïque en Aquitaine. Paris : Éd. du CNRS, XXVIIIe suppl. à Gallia Préhistoire, $393 \mathrm{p}$.

DELLUC B. et DELLUC G. 2004. L'art à l'abri Pataud (Les Eyzies, Dordogne). In M. Lejeune et A.-C. Welté (dir.), L'Art pariétal paléolithique dans son contexte naturel. Actes du colloque 8.2, Congrès de l'UISPP, Liège 2-8 sept. 2001, p. 87-94 (ERAUL, 107).

DELPORTE H. 1979 - L'image de la femme dans l'art préhistorique. Paris : Picard, $320 \mathrm{p}$.

FERUGLIO V. et BAFFIER D. 2005 - Les dessins noirs des salles Hillaire et du Crâne, grotte Chauvet-Pont-d'Arc : chronologie relative. In J.-M. Geneste dir., Recherches pluridisciplinaires dans la grotte Chauvet, Journée SPF, Lyon, 11-12 octobre 2003, Bull. de la Soc. Préhist. franç., 102,1, p. $149-158$.

FERUGLIO V., JAUBERT J., LORBLANCHET M. et coll. PEIRÉ J.-F. et MAKSUD F. 2005 - Frayssinet-le-Gélat. Grotte de Combe-Nègre. Bilan scientifique 2002. DRAC Midi-Pyrénées, Service régional de l'archéologie, Toulouse, p. 113-115.

FERUGLIO V., JAUBERT J., LORBLANCHET M. (2007) Deux sanctuaires ornés en Quercy : le réseau de CombeNègre à Frayssinet-le-Gélat (Lot). Hommages à Henri Delporte. Paris : Éd. du CTHS, p. 71-82

FOUCHER P. 2004 - Les industries lithiques du complexe Gravettien-Solutréen dans les Pyrénées. Techno-typologie et circulation des matières siliceuses de part et d'autre des Pyrénées. Toulouse : Thèse Univ. de Toulouse-Le Mirail, 3 vol., 334 p., 235 fig.

FOUCHER P. 2005 - Gargas et l'Atlantique: les relations transpyrénéennes au cours du Gravettien. In : « Homenaje a Jesús Altuna », San Sebastián : Munibe, 2005/2006, 57, p. 131-147.

FOUCHER P., SAN JUAN Cr., VALLADAS H., BÉGOUËN R., CLOTTES J. et GIRAUD J.-P. 2001 - De nouvelles dates ${ }^{14} \mathrm{C}$ pour le Gravettien des Pyrénées centrales. Préhistoire ariégeoise, Bull. de la Soc. Préhist. Arièges-Pyrénées, LVI, p. 35-44.

GENESTE J.-M. 1984 - Abri Vignaud, in J.-Ph. Rigaud dir., Informations archéologiques de la circonscription d'Aquitaine. Gallia Préhist., 27, 2, p. 274-275.

GIRARD M., BAFFIER D., TISNERAT N., VALLADAS H., ARNOLD M., HEDGES R. 1996 - Dates ${ }^{14} \mathrm{C}$ en spectrométrie de masse par accélérateur à la grande grotte d'Arcysur-Cure (Yonne). Le Paléolithique supérieur entre Seine et Rhin, Actes de la table-ronde de Dijon, p. 17-23.

GLORY Abbé A. 1960 - Les peintures de Lascaux sontelles périgordiennes ? Antiquités Nationales, II, 1960, p. 26.

GLORY Abbé A. 1965a - Nouvelles découvertes de dessins rupestres sur le Causse de Gramat (Lot). Bull. de la Soc. Préhist. Franç., LXII, p. 528-538.

GLORY Abbé A. 1965b - La grotte de Roucadour : Le panneau Il peint et gravé. Bull. de la Soc. d'Études et de Rech. Préhist. des Eyzies, juin 1966, p. 135-143. 
GUICHARD G., DELLUC B., DELLUC G. 1984 - Grotte d'Oreille d'Enfer. L'Art des Cavernes. Atlas des grottes ornées paléolithiques françaises. Paris: Ministère de la Culture, Imprimerie Nationale, p. 151-153.

GUILLERMIN P. 2008 - Les "Périgordiens" en Quercy : l'exemple du gisement des Fieux. Paleo $n^{\circ} 20$.

GUY E. 2004 - La grotte Chauvet : un art totalement homogène ? Paleoesthetique.com, fév. 2004.

HENRY-GAMBIER D. 2008 - Pratiques funéraires des populations gravettiennes en Europe : bilan des données et interprétations. Paleo, $\mathrm{n}^{\circ} 20$.

JAUBERT J. 2001 - Un nouveau sanctuaire paléolithique en Quercy : les grottes " ornées " de Combe Nègre à Frayssinet-le-Gélat (Lot). Préhistoire du Sud-Ouest, 8, 2001-2, p. 195-196.

LEMOZI A., RENAULT Ph., DAVID A. 1969 - Pech-Merle, Le Combel, Marcenac. Graz : Akademische Druku. Verlagsanstalt, 38 p., 67 pl. h.-t.

LEMOZI Chanoine A. 1965 - La Grotte Marcenac, station du paléolithique supérieur (étude comparative). Congr. Préhist. de France, Compte rendu de la XVle session, Principauté de Monaco, 28 août - 5 septembre 1959, p. 778-807.

LENOIR M. 1983 - Le Paléolithique des basses vallées de la Dordogne et de la Garonne. Bordeaux : Thèse doct. d'État ès Sciences, Université de Bordeaux I, 2 tomes, 702 p., tabl. h.-t., 445 fig. h.-t.

LEROI-GOURHAN A. 1965 - Préhistoire de l'Art occidental. Paris : Éd. Mazenod, 408 p. (coll. « L'Art des grandes civilisations ").

LEROI-GOURHAN A. 1971 - Préhistoire de l'Art occidental. Paris : $2^{\ominus}$ édit., Éd. Mazenod, 502 p. (coll. « L'Art des grandes civilisations $»)$.

LORBLANCHET M. 1970 - La Grotte des Merveilles à Rocamadour et ses peintures préhistoriques. Bull. de la Soc. d'Études Litt., Sc. et Artist. du Lot, XCl, 4, 24 p.

LORBLANCHET M. 1972 - L'Art préhistorique en Quercy : les grottes peintes et gravées. Bull. de la Soc. d'Études Litt., Sc. et Artist. du Lot, XCIII, 3, p. 27-35.

LORBLANCHET M. 1974 - L'art préhistorique en Quercy. La grotte des Escabasses (Thémines-Lot). Morlaas : Éd. P.G.P., Zaragoza, 104 p., pl. h.-t.

LORBLANCHET M. 1980 - Peindre sur les parois des grottes. Les Dossiers de l'Archéologie, 41, « Revivre la Préhistoire », p. 33-39.

LORBLANCHET M. 1981 - Fréquentation humaine et animale de la grotte du Pech-Merle, Cabrerets (Lot). Congrès
Préhist. de France, $\mathrm{XX} \mathrm{P}^{\mathrm{e}}$ Session, Montauban-Cahors, Sept. 1979, Soc. Préhist. Franç., p. 210-222 (Vol. 1, « La Préhistoire du Quercy dans le contexte de Midi-Pyrénées ").

LORBLANCHET M. 1984a - Grotte du Pech-Merle. L'Art des Cavernes. Atlas des grottes ornées paléolithiques françaises. Paris : Ministère de la Culture, Imprimerie Nationale, p. 467-474.

LORBLANCHET M. 1984b - Grotte de Roucadour. L'Art des Cavernes. Atlas des grottes ornées paléolithiques françaises. Paris : Ministère de la Culture, Imprimerie Nationale, p. 511-523.

LORBLANCHET M. 1984c - Grotte des Fieux. L'Art des Cavernes. Atlas des grottes ornées paléolithiques françaises. Paris : Ministère de la Culture, Imprimerie Nationale, p. 480-482.

LORBLANCHET M. 1984d - Grotte de Marcenac. L'Art des Cavernes. Atlas des grottes ornées paléolithiques françaises. Paris : Ministère de la Culture, Imprimerie Nationale, p. 463-466.

LORBLANCHET M. 1989 - Nouvelles découvertes d'art pariétal paléolithique en Quercy. In : L'art pariétal paléolithique. Paris : Actes des colloques de la Direction du Patrimoine, Périgueux-Le Thot, Nov. 1984, p. 79-105.

LORBLANCHET M. (Ed.) 1992 - Rock Art in the Old World. AURA Congress, Indira Gandhi National Centre For the Arts, New Delhi, 583 p.

LORBLANCHET M. 1993 - Pochoir et soufflé. In : G.R.A.P.P., L'art pariétal paléolithique. Techniques et méthodes d'étude. Paris : Éd. du C.T.H.S., p. 257-260.

LORBLANCHET M. 1994 - La datation de l'art pariétal paléolithique. Bull. de la Soc des Études Litt., Scient. et Hist. du Lot, CXV, p. 161-182.

LORBLANCHET M. 1995 - Les grottes ornées de la Préhistoire. Nouveaux regards. Paris : Éd. errance, 288 p.

LORBLANCHET M. 2004 - L'Art préhistorique du Quercy. Portet-sur-Garonne : Éd. Loubatières, 93 p.

LORBLANCHET M., LAFAITE P., BOURNAZEL S. 2005 La grotte des Fieux (Miers, Lot). CD-Rom, PROMOSAF.

LORBLANCHET M., CACHIER H., VALLADAS H. 1995 Datation d'un des chevaux du Pech-Merle. INORA, 12, p. 2-3.

LORBLANCHET M., LABEAU M., VERNET J.-L., FITTE P., VALLADAS H., CACHIER H., ARNOLD M. 1990 - Étude des pigments de grottes ornées paléolithiques du Quercy. Bull. de la Soc. des Études du Lot, 2, p. 93-143.

MOVIUS H. L. Jr. 1977 - Excavation of the Abri Pataud, Les Eyzies (Dordogne). Stratigraphy. Bull. of the American 
School of Prehistory Research, 31, Peabody Museum, Harvad University, 165 p.

NIVELLE V. 2003 - Contribution à l'étude de l'art pariétal à l'abri Pataud (Les Eyzies-de-Tayac, Dordogne). Inventaire raisonné des fragments rocheux ornés. Perpignan : Mémoire de Maîtrise, Université de Perpignan, $161 \mathrm{p}$.

PIGEAUD R. 2001 - Les représentations de la grotte ornée Mayenne-Sciences (Thoriginé-en-Charnie, Mayenne) dans leur cadre archéologique régional. Paris : Thèse Doct. Muséum National d'Histoire Naturelle, IPH, 2 vol.

PIGEAUD R. 2004 - Dialogue avec la paroi : cas des représentations paléolithiques de la grotte ornée de MayenneSciences (Thorigné-en-Charnie, Mayenne). In M. Lejeune (dir.), L'Art pariétal paléolithique dans son contexte naturel. Actes du colloque 8.2, Congrès de I'UISPP, Liège 2-8 sept. 2001, Liège, p. 21-43 (ERAUL, 107).

PIGEAUD R., VALLADAS H., ARNOLD M., CACHIER $\mathrm{H}$. 2003 - Deux datations carbone 14 en spectrométrie de masse par accélérateur (SMA) pour une représentation pariétale de la grotte Mayenne-Sciences (Thorigné-enCharnie, Mayenne) : émergence d'un art gravettien en France septentrionale ? C.R. Palevol., 2, p. 161-168.

ROQUE C., GUIBERT P., VARTARIAN E., BECHTEL Fr., OBERLIN Ch., ÉVIN J., MERCIER N., VALLADAS $H_{\text {., }}$ TEXIER J.-P., RIGAUD J.-Ph., DELPECH Fr., CLEYETMERLE J.-J., TURQ A. 2001 - Une expérience de croisement de datations $\mathrm{TL} /{ }^{14} \mathrm{C}$ pour la séquence solutréenne de Laugerie-Haute, Dordogne. In J.-N. Barrandon, P. Guibert, V. Michel dir., "Datations », XXle rencontres internat. d'Archéo. et d'Histoire d'Antibes, 19-21 oct. 2000, Éd. ADPCA, p. 217-232.

ROUSSOT A. 1984a - Abri de Laussel. L'Art des Cavernes. Atlas des grottes ornées paléolithiques françaises. Paris : Ministère de la Culture, Imprimerie Nationale, p. 167-169.

ROUSSOT A. 1984b - Grotte de Pair-non-Pair. L'Art des Cavernes. Atlas des grottes ornées paléolithiques françaises. Paris : Ministère de la Culture, Imprimerie Nationale, p. 256-262.

ROUSSOT A. 1984c - Abri du Poisson. L'Art des Cavernes. Atlas des grottes ornées paléolithiques françaises. Paris : Ministère de la Culture, Imprimerie Nationale, p. 154-156.
ROUSSOT A. 1995 - Connus et inconnus sur les femmes de Laussel. La dame de Brassempouy. Actes du colloque de Brassempouy (juillet 1994). Liège, ERAUL 74, p. 221-227.

ROUSSOT A. 2000 - La vénus à la corne et Laussel. Les chefs d'œuvre du Musée d'Aquitaine. Éd. Sud-Ouest, 32 p.

SAUVET G. 2004 - Langage préhistorique, langages de préhistoriens. In F. Audouze et N. Schlanger dir., Autour de Leroi-Gourhan : contexte et actualité d'André LeroiGourhan, Antibes : Éd. ADPCA, p. 249-270.

SONNEVILLE-BORDES D. de 1960 - Le Paléolithique supérieur en Périgord. Bordeaux : Impr. Delmas, 2 vol., 558 p., 295 fig.

VALLADAS H., CACHIER H., ARNOLD M., 1990 Application de la datation carbone 14 en spectrométrie de masse par accélérateur aux grottes ornées de Cougnac et du Pech-Merle, Lot. Bull. de la Soc. des Études du Lot, 2, p. 134-137.

VALLADAS H., CACHIER H., ARNOLD M. 1993 - New radiocarbon dates for prehistoric cave paintings at Cougnac. In: M. Lorblanchet \& P. Bahn (Eds.), Rock art studies : the post-stylistic era or where do we do we go from there ? Oxbow Monograph, 35, p. 74-76.

VALLADAS H., CLOTTES J., GENESTE J.-M., GARCIA M., ARNOLD M., CACHIER H., TISNERAT N. - 2001. Evolution of prehistoric cave art. Nature, 413, p. 479.

VALLADAS H., TISNERAT N., ARNOLD M., ÉVIN J. et OBERLIN Ch. 2001 - Les dates des fréquentations. In Clottes dir., La grotte Chauvet. L'Art des origines. Paris : Le Seuil, éd., p. 32-34.

VALLADAS H., TISNERAT N., KALTNECKER É. \& ARNOLD M. 2005 - Les datations de la grotte Cosquer. In Clottes et al., 2005, op. cit., p. 56-57.

VALLADAS H., TISNERAT-LABORDE N., CACHIER H., KALTNECKER É., ARNOLD M., OBERLIN Ch. et ÉVIN J. 2004 - Bilan des datations carbone 14 effectuées sur des charbons de bois de la grotte Chauvet. In J.-M. Geneste dir., Recherches pluridisciplinaires dans la grotte Chauvet, Journée SPF, Lyon, 11-12 octobre 2003, Bull. de la Soc. Préhist. franç., 102, 1, p. 109-113. 


aléo $X X$ Paléo $X X$ Paléo $X X$ Paléo $X X$ Paléo $X X$ Paléo $X$ aléo $X X$ Paléo $X X$ Paléo $X X$ Paléo $X X$ Paléo $X X$ Paléo aléo XX Paléo XX Paléo XX Paléo XX Paléo XX Paléo aléo XX Paléo XX Paléo XX Paléo XX Paléo XX Paléo aléo XX Paléo XX Paléo XX Paléo XX Paléo XX Paléo aléo XX Paléo XX Paléo XX Paléo XX Paléo XX Paléo aléo XX Paléo XX Paléo XX Paléo XX Paléo XX Paléo aléo XX Paléo XX Paléo XX Paléo XX Paléo XX Paléo aléo XX Paléo XX Paléo XX Paléo XX Paléo XX Paléc aléo XX Paléo XX Paléo XX Paléo XX Paléo XX Paléc aléo XX Paléo XX Paléo XX Paléo XX Paléo XX Paléo aléo $X X$ Paléo $X X$ Paléo $X X$ Paléo XX Paléo XX Paléo aléo $X X$ Paléo $X X$ Paléo $X X$ Paléo XX Paléo XX Paléc aléo $X X$ Paléo $X X$ Paléo $X X$ Paléo $X X$ Paléo $X X$ Paléc aléo XX Paléo XX Paléo XX Paléo XX Paléo XX Paléo aléo $X X$ Paléo $X X$ Paléo $X X$ Paléo $X X$ Paléo $X X$ Paléo aléo $X X$ Paléo XX Paléo XX Paléo XX Paléo XX Paléo aléo XX Paléo XX Paléo XX Paléo XX Paléo XX Paléc

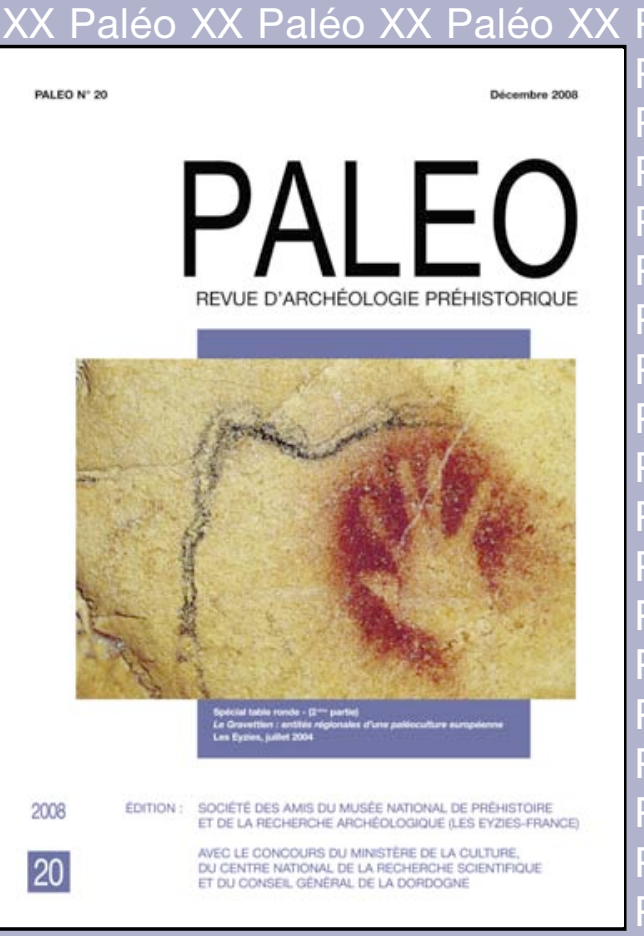
Paléo XX aléo XX Paléo XX Paléo XX Paléo XX Paléo XX Paléc Paléo $X X$ Paléo XX Paléo XX Paléo XX Paléo XX Paléo XX Paléo XX Paléo XX Paléo XX Paléo XX Paléo XX Paléo XX Paléo XX Paléo XX Paléo XX Paléo XX Paléo XX Paléo XX aléo $X X$ Paléo $X X$ Paléo $X X$ Paléo $X X$ Paléo $X X$ Paléo $X X$ Paléo $X X$ Paléo $X X$ Paléo $X X$ Paléo $X X$ aléo $X X$ Paléo $X X$ Paléo $X X$ Paléo $X X$ Paléo $X X$ Paléo $X X$ Paléo $X X$ Paléo $X X$ Paléo $X X$ Paléo $X X$ aléo $X X$ Paléo $X X$ Paléo $X X$ Paléo $X X$ Paléo $X X$ Paléo $X X$ Paléo $X X$ Paléo $X X$ Paléo $X X$ Paléo $X X$ aléo $X X$ Paléo $X X$ Paléo $X X$ Paléo $X X$ Paléo $X X$ Paléo $X X$ Paléo $X X$ Paléo $X X$ Paléo $X X$ Paléo $X X$ aléo $X X$ Paléo $X X$ Paléo $X X$ Paléo $X X$ Paléo XX Paléo XX Paléo XX Paléo XX Paléo XX Paléo XX aléo $X X$ Paléo $X X$ Paléo $X X$ Paléo $X X$ Paléo $X X$ Paléo $X X$ Paléo $X X$ Paléo $X X$ Paléo $X X$ Paléo $X X$ aléo $X X$ Paléo $X X$ Paléo $X X$ Paléo $X X$ Paléo $X X$ Paléo $X X$ Paléo $X X$ Paléo $X X$ Paléo $X X$ Paléo $X X$ aléo $X X$ Paléo $X X$ Paléo $X X$ Paléo $X X$ Paléo $X X$ Paléo $X X$ Paléo $X X$ Paléo $X X$ Paléo $X X$ Paléo $X X$ aléo $X X$ Paléo $X X$ Paléo $X X$ Paléo $X X$ Paléo $X X$ Paléo XX Paléo XX Paléo XX Paléo XX Paléo XX aléo $X X$ Paléo $X X$ Paléo $X X$ Paléo $X X$ Paléo XX Paléo XX Paléo XX Paléo XX Paléo XX Paléo XX aléo $X X$ Paléo $X X$ Paléo $X X$ Paléo $X X$ Paléo XX Paléo XX Paléo XX Paléo XX Paléo XX Paléo XX aléo $X X$ Paléo $X X$ Paléo $X X$ Paléo $X X$ Paléo $X X$ Paléo $X X$ Paléo $X X$ Paléo $X X$ Paléo $X X$ Paléo $X X$ aléo $X X$ Paléo $X X$ Paléo $X X$ Paléo $X X$ Paléo $X X$ Paléo XX Paléo XX Paléo XX Paléo XX Paléo XX aléo $X X$ Paléo $X X$ Paléo $X X$ Paléo $X X$ Paléo $X X$ Paléo XX Paléo XX Paléo XX Paléo XX Paléo XX aléo $X X$ Paléo $X X$ Paléo $X X$ Paléo $X X$ Paléo $X X$ Paléo XX Paléo XX Paléo XX Paléo XX Paléo XX aléo $X X$ Paléo $X X$ Paléo $X X$ Paléo $X X$ Paléo $X X$ Paléo $X X$ Paléo $X X$ Paléo $X X$ Paléo $X X$ Paléo $X X$ aléo XX Paléo XX Paléo XX Paléo XX Paléo XX Paléo XX Paléo XX Paléo XX Paléo XX Paléo XX aléo $X X$ Paléo $X X$ Paléo $X X$ Paléo $X X$ Paléo $X X$ Paléo XX Paléo XX Paléo XX Paléo XX Paléo XX aléo $X X$ Paléo $X X$ Paléo $X X$ Paléo $X X$ Paléo $X X$ Paléo $X X$ Paléo $X X$ Paléo $X X$ Paléo $X X$ Paléo $X X$ aléo $X X$ Paléo $X X$ Paléo $X X$ Paléo $X X$ Paléo $X X$ Paléo $X X$ Paléo $X X$ Paléo $X X$ Paléo $X X$ Paléo $X X$ aléo $X X$ Paléo $X X$ Paléo $X X$ Paléo $X X$ Paléo $X X$ Paléo XX Paléo XX Paléo XX Paléo XX Paléo XX aléo $X X$ Paléo $X X$ Paléo $X X$ Paléo $X X$ Paléo $X X$ Paléo XX Paléo XX Paléo XX Paléo XX Paléo XX aléo XX Paléo XX Paléo XX Paléo XX Paléo XX Paléo XX Paléo XX Paléo XX Paléo XX Paléo XX aléo $X X$ Paléo $X X$ Paléo XX Paléo XX Paléo XX Paléo XX Paléo XX Paléo XX Paléo XX Paléo XX aléo $X X$ Paléo $X X$ Paléo $X X$ Paléo $X X$ Paléo $X X$ Paléo XX Paléo XX Paléo XX Paléo XX Paléo XX aléo $X X$ Paléo $X X$ Paléo XX Paléo XX Paléo XX Paléo XX Paléo XX Paléo XX Paléo XX Paléo XX aléo $X X$ Paléo $X X$ Paléo $X X$ Paléo $X X$ Paléo $X X$ Paléo $X X$ Paléo $X X$ Paléo $X X$ Paléo $X X$ Paléo $X X$ aléo XX Paléo XX Paléo XX Paléo XX Paléo XX Paléo XX Paléo XX Paléo XX Paléo XX Paléo XX aléo XX Paléo XX Paléo XX Paléo XX Paléo XX Paléo XX Paléo XX Paléo XX Paléo XX Paléo XX aléo $X X$ Paléo $X X$ Paléo $X X$ Paléo $X X$ Paléo XX Paléo XX Paléo XX Paléo XX Paléo XX Paléo XX aléo $X X$ Paléo $X X$ Paléo $X X$ Paléo $X X$ Paléo $X X$ Paléo $X X$ Paléo $X X$ Paléo $X X$ Paléo $X X$ Paléo $X X$ aléo $X X$ Paléo $X X$ Paléo $X X$ Paléo $X X$ Paléo $X X$ Paléo $X X$ Paléo $X X$ Paléo $X X$ Paléo $X X$ Paléo $X X$ aléo XX Paléo XX Paléo XX Paléo XX Paléo XX Paléo XX Paléo XX Paléo XX Paléo XX Paléo XX aléo $X X$ Paléo $X X$ Paléo $X X$ Paléo $X X$ Paléo $X X$ Paléo $X X$ Paléo $X X$ Paléo $X X$ Paléo $X X$ Paléo $X X$ aléo XX Paléo XX Paléo XX Paléo XX Paléo XX Paléo XX Paléo XX Paléo XX Paléo XX Paléo XX aléo XX Paléo XX Paléo XX Paléo XX Paléo XX Paléo XX Paléo XX Paléo XX Paléo XX Paléo XX aléo $X X$ Paléo $X X$ Paléo $X X$ Paléo $X X$ Paléo $X X$ Paléo $X X$ Paléo $X X$ Paléo $X X$ Paléo $X X$ Paléo $X X$ 
